UCLA/03/TEP/31

Columbia/Math/03

15 December 2003

\title{
TWO-LOOP SUPERSTRINGS ON ORBIFOLD COMPACTIFICATIONS ${ }^{1}$
}

\author{
Kenichiro Aoki ${ }^{a}$, Eric D'Hoker ${ }^{b}$ and D.H. Phong ${ }^{c}$ \\ ${ }^{a}$ Hiyoshi Department of Physics \\ Keio University, Yokohama 223-8521, Japan \\ ${ }^{b}$ Department of Physics and Astronomy \\ University of California, Los Angeles, CA 90095, USA \\ ${ }^{c}$ Department of Mathematics \\ Columbia University, New York, NY 10027, USA
}

\begin{abstract}
The two-loop chiral measure for superstring theories compactified on $\mathbf{Z}_{2}$ reflection orbifolds is constructed from first principles for even spin structures. This is achieved by a careful implementation of the chiral splitting procedure in the twisted sectors and the identification of a subtle worldsheet supersymmetric and supermoduli dependent shift in the Prym period. The construction is generalized to compactifications which involve more general NS backgrounds preserving worldsheet supersymmetry. The measures are unambiguous and independent of the gauge slice.

Two applications are presented, both to superstring compactifications where 4 dimensions are $\mathbf{Z}_{2}$-twisted and where the GSO projection involves a chiral summation over spin structures. The first is an orbifold by a single $\mathbf{Z}_{2}$-twist; here, orbifolding reproduces a supersymmetric theory and it is shown that its cosmological constant indeed vanishes. The second model is of the type proposed by Kachru-KumarSilverstein and additionally imposes a $\mathbf{Z}_{2}$-twist by the parity of worldsheet fermion number; it is shown here that the corresponding cosmological constant does not vanish pointwise on moduli space.
\end{abstract}

\footnotetext{
${ }^{1}$ Research supported in part by a Grant-in-Aid from the Ministry of Education, Science, Sports and Culture and grants from Keio University (K.A.), and by National Science Foundation grants PHY-0140151 (E.D.) and DMS-02-45371 (D.P.).
} 


\section{Introduction}

Over the past few years, important progress has been made on two-loop superstring perturbation theory. A formula for the two-loop even spin structure superstring measure has been constructed which is well-defined and independent of any choice of gauge slice $[1,2,3,4,5]$. The independence of gauge slice choices is essential, since without it the superstring scattering amplitudes would be ambiguous. The formula has been applied to the evaluation of the two-loop $N$-point function for $N \leq 4$ massless external NS-NS string states, propagating in flat Minkowski space-time. In particular, the cosmological constant for flat Minkowski space-time had been shown to vanish point by point on moduli space.

The original derivation of these results in [4] was carried out using a gauge where the worldsheet gravitini are supported at two points $q_{1}, q_{2}$ which obey the gauge condition, $S_{\delta}\left(q_{1}, q_{2}\right)=0$, where $S_{\delta}$ is the Szegö kernel (or fermion propagator) for even spin structure $\delta$. Recently, the results of [3] (which were expressed in a general gauge where the measure is formulated in terms of meromorphic functions and forms) were used to evaluate the superstring measure in a hyper-elliptic gauge by Zheng, $\mathrm{Wu}$ and $\mathrm{Zhu}$ in [6, 7], and the vanishing of the $N \leq 3$-point functions was confirmed. The cancellations of these amplitudes are examples of non-renormalization conjectures suggested by space-time supersymmetry [8]. A bibliography of earlier work on this topic may be found in [4].

The purpose of the present paper is to derive the superstring measure (and the cosmological constant) for orbifold compactification backgrounds produced by general $\mathbf{Z}_{2}$ reflections. Certain orbifold models of this type provide some of the simplest solvable examples of superstring theories with broken supersymmetry and are therefore of considerable interest in particle physics. In particular, Kachru, Kumar and Silverstein [9] have proposed $\mathbf{Z}_{2} \times \mathbf{Z}_{2}$ orbifolds of Type II superstring theory with broken supersymmetry (see also [10]), whose 1-loop cosmological constant vanishes by construction. Clearly, the construction of any superstring theory model with broken supersymmetry and vanishing (or very small) cosmological constant would be of great phenomenological value (see also [11]).

In [9], arguments were presented in favor of the vanishing of the cosmological constant to two-loop order (and higher), using the earlier string measure suggested by the picture changing operator and BRST formalism $[12,13]$. Shortly thereafter, arguments were given against the vanishing of the two-loop cosmological constant in the same model [14], also using the picture changing operator and BRST formalism. In both approaches, special choices for the worldsheet gravitini insertion points were made, the first in unitary gauge, the second following [15]. By now, however, the picture changing operator and BRST formalism is well-known to be gauge slice dependent [13], (see also [16, 17, 18, 19]) a fact that casts doubts over the conclusions of both [9] and [14].

A reliable calculation of the cosmological constant requires a gauge slice independent measure such as the one obtained in $[1,2,3,4]$ for flat Minkowski space-time. 
In the present paper, the two-loop chiral measure for superstring theories compactified on $\mathbf{Z}_{2}$ reflection orbifolds is constructed from first principles for even spin structures. This is achieved by a careful implementation of the chiral splitting procedure in the twisted sectors and the identification of a subtle worldsheet supersymmetric and supermoduli dependent shift in the Prym period. The limits of the measure are checked under various degenerations and are found to agree with the behavior expected on physical grounds.

The construction is generalized to compactifications which involve more general NS backgrounds, and preserve worldsheet supersymmetry. The measures are shown to be unambiguous and independent of the gauge slice. Our original primary concern was with the study of the models of [9]. The results obtained in this paper for the $\mathbf{Z}_{2}$-twisted chiral measure, however, will be indispensable ingredients in the construction of the $\mathbf{Z}_{2}$-twisted conformal field theory and string amplitudes to two-loop order in any orbifold model based on $\mathbf{Z}_{2}$ reflections.

Two applications are presented, both to superstring compactifications where 4 dimensions are $\mathbf{Z}_{2}$-twisted and where the GSO projection involves a chiral summation over spin structures, i.e. a summation carried out independently on left and right movers. The first is an orbifold by a single $\mathbf{Z}_{2}$ twist, which is known to reproduce a supersymmetric theory; it is shown here that its cosmological constant vanishes. The second model is of the type proposed by Kachru-Kumar-Silverstein [9] and additionally imposes a $\mathbf{Z}_{2}$ twist by the parity of worldsheet fermion number; it is shown here that the corresponding chiral measure is non-vanishing. Therefore, the associated cosmological constant does not vanish pointwise on moduli space. It is logically possible, though we shall argue it is unlikely, that despite this result, a cancellation will occur for the cosmological constant when left and right chiral blocks are assembled and the integration over moduli is carried out.

\subsection{Outline of the construction}

In $[1,2,3,4]$, a detailed method was laid out for determining the two-loop superstring measure on general space-times. Explicit formulas were obtained in those papers for the case of flat Minkowski space-time $M$; they involve the matter and ghost supercurrents, stress tensors, and partition functions.

All compactifications discussed in this paper will only affect the matter fields and will leave the ghost fields unchanged. In orbifold compactifications $C$ of flat Minkowski spacetime, the expressions for the matter supercurrent and stress tensor (in terms of the fields $x^{\mu}$ and $\left.\psi_{ \pm}^{\mu}\right)$ are identical to those of flat Minkowski space-time and will be denoted by $S_{M m}$ and $T_{M m}$ (or simply $S_{m}$ and $T_{m}$ when no confusion is possible). The fields $x^{\mu}$ and $\psi_{ \pm}^{\mu}$ will be summed over untwisted and twisted sectors, dependent on $C$. Therefore, the partition function, and more generally any vacuum expectation value will depend on $C$; they will be referred to by $Z_{C}$ and $\langle\cdots\rangle_{C}$. (On a more general compactification background $C$, the 
expression for the supercurrent and stress tensor (in terms of $x^{\mu}$ and $\psi_{ \pm}^{\mu}$ ) will generally be different from their expressions in flat space-time and will be denoted by $S_{C m}$ and $T_{C m}$.)

Thus, the main new ingredients are the construction of the propagators for the $\mathbf{Z}_{2^{-}}$ twisted fields $x^{\mu}$ and $\psi_{ \pm}^{\mu}$, and the identification of the corresponding changes in the string measure. These changes reflect the global geometry of the worldsheet with a quadratic branch cut, and must be treated with care. We summarize here the main steps in our derivation.

- The first step is to determine the $\mathbf{Z}_{2}$-twisted bosonic propagator $\left\langle\partial_{z} x(z) \partial_{w} x(w)\right\rangle_{\varepsilon}$ for given twist $\varepsilon$. Its explicit expression will be given in terms of the familiar Szegö kernel, as well as the Prym period $\tau_{\varepsilon}$ and the Prym differential $\omega_{\varepsilon}$ for twist $\varepsilon$. The propagator for the $\mathbf{Z}_{2}$-twisted fermionic field $\psi_{+}$was identified in [9].

- The next step is to carry out chiral splitting for the $\mathbf{Z}_{2}$-twisted matter superfields. Since the $\mathbf{Z}_{2}$-twisted propagators are different from those of flat space-time, the effective rules, derived in $[20,21]$ for Minkowski space-time, will not apply directly in this case. The $\mathbf{Z}_{2}$-twisted case has to be worked out in its own right, and its own global geometric features carefully accounted for. In fact, it is here that a subtle and crucial supersymmetric correction $\Delta \tau_{\varepsilon}$ to the super Prym period makes its appearance;

- An important check is the independence of the gauge-fixed measure from the choice of gauge slice. In fact, a formula will be derived for the two-loop measure in terms of the partition function, supercurrent and stress tensor correlators for any compactification that does not affect the ghosts, preserves worldsheet supersymmetry and has matter central charge $c=15$. The resulting measure will be shown to be unambiguous and slice independent under these assumptions. This result was announced in [1].

- The next task is to express the $\mathbf{Z}_{2}$-twisted superstring measure in terms of $\vartheta$ constants. The procedure follows $[1,2,3,4]$, but here there is a significant new difficulty. A new, internal momentum $p_{\varepsilon}$-dependent, contribution arises from the correction $\Delta \tau_{\varepsilon}$ to the super Prym period. The explicit evaluation, in terms of $\vartheta$-constants, of this contribution is one of the more arduous steps in the paper. The resulting chiral superstring measure depends on both the spin structure $\delta$ and the twist $\varepsilon$ and is denoted $d \mu_{C}[\delta ; \varepsilon]\left(p_{\varepsilon}\right)$.

- The limit of the chiral measure $d \mu_{C}[\delta ; \varepsilon]\left(p_{\varepsilon}\right)$ is computed for a variety of degenerations and checked versus the limiting behavior expected on physical grounds. The limits will also be needed to investigate the behavior of the cosmological constant.

- Next, the behavior of the chiral measure $d \mu_{C}[\delta ; \varepsilon]\left(p_{\varepsilon}\right)$ under modular transformations is derived. The full genus 2 modular group $S p(4, \mathbf{Z})$ will act on the twist cycle and relate the measures for various twists. In carrying out the GSO projection, a summation over all spin structures is to be carried out, for fixed twist. Therefore, it is the subgroup $H_{\varepsilon}$ of all elements in $S p(4, \mathbf{Z})$ which leave $\varepsilon$ invariant which will be of greatest importance in 
determining all possible GSO projections consistent with modular covariance of the chiral measure. The group $H_{\varepsilon}$ is studied in detail; it is found to be generated by 5 independent elements (while $S p(4, \mathbf{Z})$ is generated by 6 ), the various orbits under $H_{\varepsilon}$ for twists and spin structures are calculated, and the behavior of $d \mu_{C}[\delta ; \varepsilon]\left(p_{\varepsilon}\right)$ is obtained.

- As a general principle, the GSO projection is carried out by summing over the spin structures $\delta$ with suitable phases. In practice, for each specific physical model, the conformal blocks entering the chiral string measure have to be identified and summed over $\delta$ consistently with modular covariance. For the simplest $\mathbf{Z}_{2}$-twisted theories, where the orbifold group is exactly $\mathbf{Z}_{2}$ acting by reflection, the conformal blocks are just $d \mu_{C}[\delta ; \varepsilon]\left(p_{\varepsilon}\right)$. However, for more complicated orbifold groups such as $\mathbf{Z}_{2} \times \mathbf{Z}_{2}$ in the models of [9], the conformal blocks can be more subtle, and have to be determined with some care. The cosmological constant for various asymmetric $\mathbf{Z}_{2}$-orbifold models is then determined by summing over the spin structures $\delta$. In particular, the cosmological constant in the models of Kachru-Kumar-Silverstein [9] is shown to be non-vanishing pointwise on moduli space.

\subsection{Organization and Summary of Main Formulas}

In section $\S 2$, standard facts about conformal field theory on genus 1 and genus 2 Riemann surfaces as well as the partition function of a $\mathbf{Z}_{2}$-twisted boson [22] are reviewed. A selfcontained construction is presented of the Prym differential $\omega_{\varepsilon}(z)$, the Prym period $\tau_{\varepsilon}$ and the Schottky relations. ${ }^{1}$ The key result in section $\S 2$ is for the $\mathbf{Z}_{2}$-twisted boson propagator on a genus 2 Riemann surface $\Sigma$. In a canonical homology basis with cycles $A_{I}, B_{I}, I=1,2$, the 16 possible twists may be described in terms of half-characteristics $\varepsilon=\left(\varepsilon_{I}^{\prime}, \epsilon_{I}^{\prime \prime}\right)$. The bosonic field $x(z)$ in twist sector $\varepsilon$ satisfies the following boundary conditions,

$$
x\left(z+A_{I}\right)=(-)^{2 \varepsilon_{I}^{\prime}} x(z), \quad x\left(z+B_{I}\right)=(-)^{2 \varepsilon_{I}^{\prime \prime}} x(z)
$$

The corresponding $\mathbf{Z}_{2}$-twisted propagator is characterized by the same boundary conditions in both variables $z$ and $w$, and the short distance singularity $(z-w)^{-2}$. It is obtained in terms of the Szegö kernel $S_{\delta}$ for characteristic $\delta$, and the normalized Prym differential $\omega_{\varepsilon}$,

$$
\left\langle\partial_{z} x(z) \partial_{w} x(w)\right\rangle_{\varepsilon}=S_{\delta_{i}^{+}}(z, w) S_{\delta_{i}^{-}}(z, w)-4 \pi i \partial_{\tau_{\varepsilon}} \ln \vartheta_{i}\left(0, \tau_{\varepsilon}\right) \omega_{\varepsilon}(z) \omega_{\varepsilon}(w)
$$

The notation here is as follows. Given a twist $\varepsilon \neq 0$, six of the ten even spin structures $\delta$ are such that $\delta+\varepsilon$ is also an even spin structure. These six spin structures may be parametrized by $\delta_{i}^{ \pm}$, with $i=2,3,4$ and $\delta_{i}^{-}=\varepsilon+\delta_{i}^{+}$and $\delta_{i}^{ \pm}$modulo $\varepsilon$ clearly map onto the 3 even spin structures of genus 1 . The $\vartheta_{i}$ are the corresponding genus $1 \vartheta$-functions for even spin structures. The precise normalization of $\omega_{\varepsilon}(z)$ is derived in $\S 2.6$.

\footnotetext{
${ }^{1}$ Detailed discussions of genus $1 \vartheta$-functions and genus 2 spin structures, $\vartheta$-functions, modular transformations and Thomae-type formulas are collected in Appendices A and B respectively.
} 
In section $\S 3$, the chiral splitting procedure is carefully implemented in the twisted sectors of the $\mathbf{Z}_{2}$-twisted superstring theory. Because of the $\mathbf{Z}_{2}$-twisting, only a single internal loop momentum survives (instead of the two internal loop momenta in the untwisted sector). This is illustrated in Figure 1, where a quadratic branch cut is applied along the cycle $C_{\varepsilon}$ producing double-valued behavior along cycle $D_{\varepsilon}$. The only internal loop momentum left crosses the remaining independent cycle $A_{\varepsilon}$.

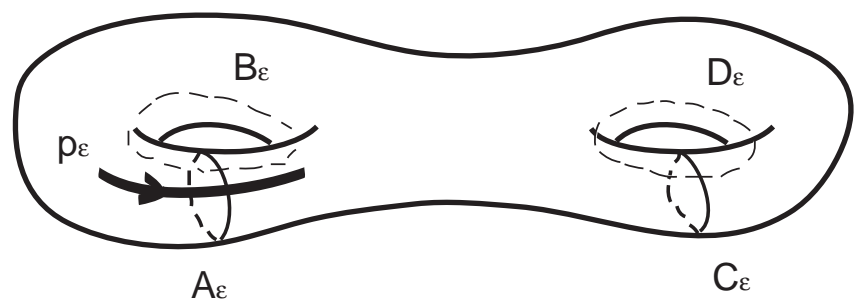

Figure 1: Twisted Cycles $A_{\varepsilon}$ and $B_{\varepsilon}$ and the single internal loop momentum $p_{\varepsilon}$

A subtle worldsheet supersymmetric and supermoduli dependent shift in the Prym period is identified. The results are as follows: let $\mathbf{A}_{C}[\delta]$ be the left-right symmetric compactified amplitude for fixed spin structure $\delta$ and $n$ directions twisted by $\mathbf{Z}_{2}$. For simplicity, only the 0-point function is considered here. It is well-known (c.f. [23], p. 967) that the gauge-fixed expression for $\mathbf{A}_{C}[\delta]$ as an integral over supermoduli space is

$$
\mathbf{A}_{C}[\delta]=\int_{s \mathcal{M}_{2}} \prod_{A}\left|d m^{A}\right|^{2} \int D(X B \bar{B} C \bar{C}) \prod_{A}\left|\delta\left(\left\langle H_{A} \mid B\right\rangle\right)\right|^{2} e^{-I_{m}-I_{g h}}
$$

Here $m^{A}$ are parameters for a $(3 \mid 2)$-dimensional slice for the even and odd supermoduli, $H_{A}$ is the corresponding super Beltrami differentials, $X, B, \bar{B}, C, \bar{C}$ are respectively the matter and ghost superfields, and $I_{m}, I_{g h}$ are respectively the matter and the ghost actions. This expression is non-chiral, but one of its fundamental properties is that it can be split as an integral over internal momenta of the norms squared of a chiral amplitude, $\mathcal{A}_{C}[\delta, \varepsilon]\left(p_{\varepsilon}\right)$,

$$
\mathbf{A}_{C}[\delta]=\sum_{\varepsilon} \int d^{10-n} p_{I} \int d^{n} p_{\varepsilon} \int_{s \mathcal{M}_{2}} \prod_{A}\left|d m^{A}\right|^{2}\left|\mathcal{A}_{C}[\delta, \varepsilon]\left(p_{\varepsilon}\right)\right|^{2} \mid e^{\left.i \pi p_{I}^{\mu} \hat{\Omega}_{I J} p_{J}^{\mu}\right|^{2}}
$$

The chiral amplitude $\mathcal{A}_{C}[\delta, \varepsilon]\left(p_{\varepsilon}^{\mu}\right)$ is superholomorphic on supermoduli space $s \mathcal{M}_{2}$ and may be computed in terms of the chiral partition functions $Z_{C}$ and $Z_{M}$ of the compactified and Minkowskian theories respectively, following the method of $[1,2,3,4]$. Parametrizing the genus 2 supermoduli space by the super period matrix $\hat{\Omega}_{I J}$ as bosonic coordinates and the 
two Grassmann coordinates $\zeta^{1}, \zeta^{2}$ as fermionic coordinates, one obtains,

$$
\begin{aligned}
\mathcal{A}_{C}[\delta, \varepsilon]\left(p_{\varepsilon}\right)= & \mathcal{A}_{M}[\delta] \frac{Z_{C}[\delta ; \varepsilon]}{Z_{M}[\delta]} \exp \left\{i \pi\left(\hat{\tau}_{\varepsilon}+\Delta \tau_{\varepsilon}\right) p_{\varepsilon}^{2}\right\} \\
& \times\left\{1-\frac{1}{8 \pi^{2}} \int d^{2} z \int d^{2} w \chi_{\bar{z}}^{+} \chi_{\bar{w}}^{+}\left(\left\langle S_{m}(z) S_{m}(w)\right\rangle_{C}-\left\langle S_{m}(z) S_{m}(w)\right\rangle_{M}\right)\right. \\
& \left.\quad+\frac{1}{2 \pi} \int d^{2} z \hat{\mu}(z)\left(\left\langle T_{m}(z)\right\rangle_{C}-\left\langle T_{m}(z)\right\rangle_{M}\right)\right\}
\end{aligned}
$$

Here, the expression $\mathcal{A}_{M}[\delta]$ is the chiral superstring measure of the Minkowski space theory, which was evaluated in $[1,4]$. The expressions $Z_{M}[\delta]$ and $Z_{C}[\delta, \epsilon]$ are the matter partition functions for the flat Minkowski space theory $(M)$ and the $\mathbf{Z}_{2}$-twisted theory $(C)$. The expressions $S_{m}$ and $T_{m}$ are the matter supercurrent and stress tensor respectively. The bosonic Prym period $\tau_{\varepsilon}$ is related to the bosonic period matrix $\Omega$, a relation that we shall denote by $\tau_{\varepsilon}=R_{\varepsilon}(\Omega)$, and is given explicitly by (for any $i, j=2,3,4$ )

$$
\tau_{\varepsilon}=R_{\varepsilon}(\Omega) \quad \Leftrightarrow \quad \frac{\vartheta_{i}\left(0, \tau_{\varepsilon}\right)^{4}}{\vartheta_{j}\left(0, \tau_{\varepsilon}\right)^{4}}=\frac{\vartheta\left[\delta_{i}^{+}\right](0, \Omega)^{2} \vartheta\left[\delta_{i}^{-}\right](0, \Omega)^{2}}{\vartheta\left[\delta_{j}^{+}\right](0, \Omega)^{2} \vartheta\left[\delta_{j}^{-}\right](0, \Omega)^{2}}
$$

The super-Prym period $\hat{\tau}_{\varepsilon}$ and the super-period matrix $\hat{\Omega}_{I J}$ are related via this same equation $\hat{\tau}_{\varepsilon}=R_{\varepsilon}(\hat{\Omega})$ and both are invariant under local worldsheet supersymmetry. The quantity $\Delta \tau_{\varepsilon}$ is a subtle correction which arises from chiral splitting and is given by ${ }^{2}$

$$
\Delta \tau_{\varepsilon}=-\frac{i}{8 \pi} \int d^{2} z \int d^{2} w \chi_{\bar{z}}^{+} S_{\delta}(z, w) \chi_{\bar{w}}^{+}\left\{\omega_{\varepsilon}(z) \omega_{\varepsilon}(w)-\omega_{I}(z) \omega_{J}(w) \partial_{I J} R_{\varepsilon}(\hat{\Omega})\right\}
$$

It is locally supersymmetric and non-vanishing. In the expression for $\mathcal{A}_{C}[\delta ; \varepsilon]\left(p_{\varepsilon}\right)$, all dependence on the original period matrix $\Omega_{I J}$ has been eliminated in favor of $\hat{\Omega}_{I J}$. Henceforth, we view $\hat{\Omega}_{I J}$ as the true moduli, and write it simply as $\Omega_{I J}$.

In section $\S 4$, a generalization is given for the chiral measure in an arbitrary background $C$ with worldsheet supersymmetry and matter central charge 15 . In the split gauge defined by $S_{\delta}\left(q_{1}, q_{2}\right)=0$, this expression is evaluated for each chiral sector, denoted by $\lambda$, and takes the simple form,

$$
\mathcal{A}_{C}[\delta ; \lambda]=\mathcal{A}_{M}[\delta] \frac{Z_{C}[\delta ; \lambda]}{Z_{M}[\delta]}\left\{1-\frac{\zeta^{1} \zeta^{2}}{4 \pi^{2}} \mathcal{Z}\left\langle S_{C}\left(q_{1}\right) S_{C}\left(q_{2}\right)\right\rangle_{C \lambda}\right\} .
$$

where $Z_{C}[\delta ; \lambda]$ is the matter partition function of the compactified theory $(C)$ for spin structure $\delta$ and chiral sector $\lambda$, while $S_{C}$ is the supercurrent of the compactified theory. This last formula was announced in [1]. It shows that the superstring measure for an

\footnotetext{
${ }^{2}$ Throughout, we shall use the notation $\partial_{I I} \equiv \partial / \partial \Omega_{I I}, \partial_{I J} \equiv \frac{1}{2} \partial / \partial \Omega_{I J}$ when $I \neq J$. The heat-kernel equation for the $\vartheta$-function is then simply $4 \pi i \partial_{I J} \vartheta[\delta](\zeta, \Omega)=\partial_{I} \partial{ }_{J} \vartheta[\delta](\zeta, \Omega)$, where $\partial_{I} \equiv \partial / \partial \zeta^{I}$.
} 
arbitrary background reduces to the evaluation of the matter partition function $Z_{C}$ and the supercurrent correlator $\left\langle S_{C}\left(q_{1}\right) S_{C}\left(q_{2}\right)\right\rangle_{C \lambda}$ in sector $\lambda$.

In section $\S 5$, the two-loop $\mathbf{Z}_{2}$-twisted blocks $\mathcal{A}_{C}[\delta ; \varepsilon]\left(p_{\varepsilon}\right)$ are computed in terms of $\vartheta$-functions, and a final expression for the twisted chiral measure is obtained in terms of the super-period matrix $\hat{\Omega}_{I J}$ (recall that it is now denoted simply by $\Omega_{I J}$; similarly, the super-Prym period $\hat{\tau}_{\varepsilon}$ is denoted simply by $\tau_{\varepsilon}$ ). Defining ${ }^{3}$

$$
\begin{aligned}
d \mu_{C}[\delta ; \varepsilon]\left(p_{\varepsilon}\right) & \equiv d^{3} \Omega \int d^{2} \zeta \mathcal{A}_{C}[\delta ; \varepsilon]\left(p_{\varepsilon}\right) \\
\Gamma[\delta ; \varepsilon] & \equiv \mathcal{Z} \int d \zeta^{2} d \zeta^{1} \Delta \tau_{\varepsilon}
\end{aligned}
$$

we find

$$
d \mu_{C}[\delta ; \varepsilon]\left(p_{\varepsilon}\right)=e^{i \pi \tau_{\varepsilon} p_{\varepsilon}^{2}} \frac{Z_{C}[\delta, \varepsilon]}{Z_{M}[\delta]}\left\{\frac{\Xi_{6}[\delta] \vartheta[\delta]^{4}}{16 \pi^{6} \Psi_{10}}+\left(i \pi p_{\varepsilon}^{2}-n \partial_{\tau_{\varepsilon}} \ln \vartheta_{i}\left(0, \tau_{\varepsilon}\right)\right) \Gamma[\delta ; \varepsilon]\right\} d^{3} \Omega
$$

The expression $\Xi_{6}[\delta](\Omega)$ is the modular covariant form found in $[1,2,3,4]$. The key new expression here is $\Gamma[\delta ; \varepsilon]$, which requires a substantial calculation, especially the determination of its overall sign. The final result is

$$
\Gamma\left[\delta_{i}^{ \pm}, \varepsilon\right]= \pm\left\langle\nu_{0} \mid \mu_{i}\right\rangle \frac{i}{(2 \pi)^{7}} \frac{\vartheta_{i}^{4}}{\eta^{12}}\left(\frac{\vartheta\left[\delta_{i}^{ \pm}\right]^{2}}{\vartheta\left[\delta_{i}^{\mp}\right]^{2}}\right)\left(\frac{\vartheta_{j}^{4}}{\vartheta\left[\delta_{j}^{+}\right]^{2} \vartheta\left[\delta_{j}^{-}\right]^{2}}\right)
$$

Here, the \pm signs are correlated with one another on both sides of the equation. The $\vartheta_{i}$ 's are genus $1 \vartheta$-constants with respect to $\tau_{\varepsilon}$, while the $\vartheta[\delta]$ 's are genus $2 \vartheta$-constants with characteristic $\delta$. The above expression for $\Gamma\left[\delta_{i}^{ \pm}, \varepsilon\right]$ is independent of $j$.

In section $\S 6$, modular properties in the presence of a twist are studied. The subgroup $H_{\varepsilon}$ that fixes a twist $\varepsilon$ is determined: it has 48 elements, and is of the form

$$
H_{\varepsilon}=\left\{I, T_{2}, S_{2}, S_{2} T_{2}, S_{2}^{2}, S_{2}^{2} M_{1} T_{2}\right\} \times H_{\varepsilon}^{0}
$$

The matrices $M_{1}, M_{2}, M_{3}, S_{2}, T_{2}$ are given in Appendix B.3; and $H_{\varepsilon}^{0}$ is the Abelian subgroup consisting of the elements $H_{\varepsilon}^{0}=\left\{I, M_{1}, M_{2}, M_{3}, M_{1} M_{2}, M_{1} M_{3}, M_{2} M_{3}, M_{1} M_{2} M_{3}\right\}$. The orbits under this group of both even spin structures and twists are worked out.

In section $\S 7$, some of the degeneration limits of the measure are worked out explicitly and checked versus the results expected on physical grounds.

In section $\S 8$, applications to physically interesting models are studied. These models are obtained as orbifolds of flat Minkowski space-time with symmetric or asymmetric

\footnotetext{
${ }^{3}$ The volume element is defined as follows $d^{3} \Omega=d \Omega_{11} d \Omega_{12} d \Omega_{22}$.
} 
orbifold groups $G$. The orbifold groups considered here are simply generated by reflections and shifts. The procedure of chiral splitting for symmetric orbifolds is reviewed.

A prescription is given for the construction of the string amplitudes for asymmetric orbifolds in terms of the chiral blocks obtained by chirally splitting symmetric orbifold amplitudes. The prescription proceeds as follows. For each model, the set of chiral blocks $\left\{d \mu_{L}\left[\delta_{L}, \lambda_{L}\right]\left(p_{L}\right)\right\}$, for all left chiral sectors $\lambda_{L}$, is determined from the symmetric orbifold theory obtained from the group generated by all symmetric elements of the form $\left(f_{L}, f_{L}\right)$, where $f_{L}$ runs over left elements of group elements $f=\left(f_{L}, f_{R}\right) \in G$. Similarly, the set of antiholomorphic blocks $\left\{d \mu_{R}\left[\delta_{R}, \lambda_{R}\right]\left(p_{R}\right)\right\}$ for all right chiral sectors $\lambda_{R}$, is determined from the symmetric orbifold group generated by all $\left(f_{R}, f_{R}\right)$. The GSO projection is always assumed to be implemented by carrying out a chiral summation over all spin structures independently for left and right movers,

$$
d \mu_{L}\left[\lambda_{L}\right]\left(p_{L}\right)=\sum_{\delta_{L}} \eta_{L}\left[\delta_{L} ; \lambda_{L}\right] d \mu_{L}\left[\delta_{L}, \lambda_{L}\right]\left(p_{L}\right)
$$

for suitable phases $\eta_{L}\left[\delta_{L} ; \lambda_{L}\right]$. The partition function for the asymmetric orbifold model $G$ is then given by

$$
Z_{G}=\int(\operatorname{det} \operatorname{Im} \Omega)^{-5+\frac{n}{2}} \sum_{p_{L}, p_{R}} \sum_{\lambda_{L}, \lambda_{R}} d \mu_{L}\left[\lambda_{L}\right]\left(p_{L}\right) \wedge \overline{d \mu_{R}\left[\lambda_{R}\right]\left(p_{R}\right)} K\left(\lambda_{L}, \lambda_{R} ; p_{L}, p_{R}\right)
$$

where $n$ is the number of compactified dimensions, and $K\left(\lambda_{L}, \lambda_{R} ; p_{L}, p_{R}\right)$ are suitable constant coefficients, depending only on the labels $\lambda_{L}, \lambda_{R}, p_{L}, p_{R}$ for left and right chiral blocks. The determination of $K$ is a difficult issue, and we shall discuss it further in a forthcoming publication [24]. In this paper, we shall examine only the chiral models, that is, only the issue of vanishing of the chiral blocks $d \mu_{L}\left[\lambda_{L}\right]\left(p_{L}\right)$ and $d \mu_{R}\left[\lambda_{R}\right]\left(p_{R}\right)$.

We carry out this procedure for the simplest $\mathbf{Z}_{2}$ models, where $n=4$ and $G$ is given by $\mathbf{Z}_{\mathbf{2}}$ reflections. The chiral blocks of this theory are of course just the conformal blocks $d \mu_{C}[\delta ; \varepsilon]\left(p_{\varepsilon}\right)$, with index $\lambda=\lambda_{L}=\varepsilon$, of the $\mathbf{Z}_{2}$ theory we have just derived in (1.10). It is shown that there is a unique choice of phases for each $\varepsilon$ that is consistent with modular covariance, given by

$$
\eta[\delta ; \varepsilon]=1
$$

Upon carrying out the GSO projection, the term involving $\Gamma[\delta ; \varepsilon]$ cancels due to the genus 1 Riemann identities and the chiral block reduces to

$$
d \mu_{C}[\varepsilon]\left(p_{L}\right)=\frac{e^{i \pi \tau_{\varepsilon} p_{L}^{2}}}{16 \pi^{6} \Psi_{10}} \frac{\vartheta\left[\delta_{j}^{+}\right]^{2} \vartheta\left[\delta_{j}^{-}\right]^{2}}{\vartheta_{j}^{4}} d^{3} \Omega \sum_{\delta} \Xi_{6}[\delta] \vartheta[\delta]^{2} \vartheta[\delta+\varepsilon]^{2}
$$

This expression can be shown to vanish identically, by using identities which involve $\Xi_{6}[\delta]$ and which were established in [4]. Thus the cosmological constant vanishes point by point 
on moduli space, in agreement with the fact that the theory just reproduces Type II superstrings.

The more complicated Kachru-Kumar-Silverstein models are constructed in terms of an asymmetric orbifold group, whose point group $P_{G}$ is isomorphic to $\mathbf{Z}_{2} \times \mathbf{Z}_{2}$. It is shown that the chiral blocks $d \mu_{C}[\delta ; \lambda]\left(p_{L}\right)$ of such theories are indexed by two twists $\lambda=\lambda_{L}=(\varepsilon, \alpha)$, and given explicitly by

$$
d \mu_{C}[\delta ; \lambda]\left(p_{L}\right)=\langle\alpha \mid \delta\rangle d \mu_{C}[\delta ; \varepsilon]\left(p_{L}\right)
$$

where the expression $d \mu_{C}[\delta ; \varepsilon]\left(p_{L}\right)$ on the right hand side are the $\mathbf{Z}_{2}$ blocks of (1.10). It is shown that modular covariance requires that the GSO projection phases be given by $\eta_{L}[\delta ; \lambda]=\langle\alpha \mid \delta\rangle$, that is, the chiral blocks of the theory are given by

$$
\begin{aligned}
d \mu_{C}[\lambda]\left(p_{L}\right) & =\sum_{\delta}\langle\alpha \mid \delta\rangle d \mu_{C}[\delta ; \varepsilon]\left(p_{L}\right) \\
& =\frac{e^{i \pi \tau_{\varepsilon} p_{L}^{2}}}{16 \pi^{6} \Psi_{10}} \frac{\vartheta\left[\delta_{j}^{+}\right]^{2} \vartheta\left[\delta_{j}^{-}\right]^{2}}{\vartheta_{j}^{4}} d^{3} \Omega \sum_{\delta}\langle\alpha \mid \delta\rangle \Xi_{6}[\delta] \vartheta[\delta]^{2} \vartheta[\delta+\varepsilon]^{2}
\end{aligned}
$$

For each $\varepsilon$, the label $\alpha$ in $\lambda$ can be classified into orbits of the modular group $H_{\varepsilon}$ leaving $\varepsilon$ invariant. There are 4 such orbits.

We find that the contributions of two orbits vanish identically, the contribution of a third orbit vanishes of order $\tau^{4}$ along the divisor of separating nodes, ${ }^{4}$ but the contribution of the remaining orbit vanishes only to order $\tau^{2}$ along the same divisor. More precisely, we find

$$
\begin{aligned}
& \sum_{\delta}\langle\alpha \mid \delta\rangle \Xi_{6}[\delta] \vartheta[\delta]^{2} \vartheta[\delta+\varepsilon]^{2} \\
& \quad=-256 \pi^{2} \tau^{2} \eta\left(\tau_{1}\right)^{12} \eta\left(\tau_{2}\right)^{12}\left(\vartheta_{3}^{8}-\vartheta_{4}^{8}\right)\left(\tau_{1}\right) \vartheta_{2}^{4} \vartheta_{3}^{2} \vartheta_{4}^{2}\left(\tau_{2}\right)+\mathcal{O}\left(\tau^{4}\right)
\end{aligned}
$$

for $\varepsilon$ and $\alpha$ given by

$$
\varepsilon=\left(\begin{array}{c|c}
0 & 0 \\
0 & \frac{1}{2}
\end{array}\right), \quad \alpha=\left(\begin{array}{c|c}
0 & \frac{1}{2} \\
0 & 0
\end{array}\right) \text {. }
$$

Thus the cosmological constant in the KKS models does not vanish point by point on moduli space.

\section{$2 \quad \mathrm{Z}_{2}$ Orbifold conformal field theory}

The main goal of this section is to derive elements from the theory of $\mathbf{Z}_{2}$-twisted fields which we need. For this, we require some background on spin structures, $\vartheta$-functions, double

\footnotetext{
${ }^{4}$ For brevity, the notation $\Omega_{11}=\tau_{1}, \Omega_{22}=\tau_{2}$ and $\Omega_{12}=\tau$ will be used throughout.
} 
covers, and Prym differentials, summarized in $\S 2.1$ and $\S 2.2$. The partition function for $\mathbf{Z}_{2}$-twisted bosonic scalar fields has been obtained by Dijkgraaf, Verlinde, and Verlinde [22]. It is recalled in $\S 2.3$. For our purpose, the main objects of interest are the propagators. The fermionic one is easy to find, so we concentrate on the bosonic propagator $\left\langle\partial_{z} x(z) \partial_{w} x(w)\right\rangle_{\varepsilon}$. As a warm-up, it is derived for the case of genus 1 in $\S 2.4$. The rest of the section is devoted to extending this method to the case of genus $h=2$. The Schottky relations between $\vartheta$ functions and the Prym differential are also derived.

\subsection{Spin structures and $\vartheta$-functions}

Let $\Sigma$ be an orientable compact genus $h$ Riemann surface without boundary on which a canonical homology basis for $H_{1}(\Sigma, \mathbf{Z})$ of cycles $A_{I}, B_{I}, I=1, \cdots . h$, is chosen,

$$
\#\left(A_{I}, A_{J}\right)=\#\left(B_{I}, B_{J}\right)=0 \quad \#\left(A_{I}, B_{J}\right)=-\#\left(B_{J}, A_{I}\right)=\delta_{I J}
$$

Holomorphic Abelian differentials $\omega_{I}$ may be normalized on $A$-cycles and their integral along $B$-cycles yields the period matrix $\Omega_{I J}$,

$$
\oint_{A_{I}} \omega_{J}=\delta_{I J} \quad \oint_{B_{I}} \omega_{J}=\Omega_{I J}
$$

Given the intersection numbers (2.1), the basis is unique up to modular transformations belonging to the modular group $S p(2 h, \mathbf{Z})$. The structure of the modular group, its action on spin structures, twists and $\vartheta$-functions is summarized in Appendix B.3.

A spinor field on $\Sigma$ requires the assignment of a spin structure on $\Sigma$, corresponding to an element of $H_{1}\left(\Sigma, \mathbf{Z}_{2}\right)$. In a given homology basis, a spin structure may be labeled by a half-integer characteristics $\kappa \equiv\left(\kappa^{\prime} \mid \kappa^{\prime \prime}\right)$ where $\kappa^{\prime}$ and $\kappa^{\prime \prime}$, each valued in $\left\{0, \frac{1}{2}\right\}^{h}$, and specifies the monodromies around $A$ - and $B$-cycles respectively. The parity of the spin structure $\kappa$ is that of the integer $4 \kappa^{\prime} \cdot \kappa^{\prime \prime}$, and is modular invariant. For genus 1 , the 4 spin structures separate into 3 even and 1 odd (respectively denoted $\mu$ and $\nu_{0}$ ). For genus 2 , the 16 spin structures separate into 10 even and 6 odd spin structures (respectively denoted by $\delta$ and $\nu$ ). An important pairing between characteristics is given by the signature,

$$
\langle\kappa \mid \rho\rangle \equiv \exp \left\{4 \pi i\left(\kappa^{\prime} \cdot \rho^{\prime \prime}-\rho^{\prime} \cdot \kappa^{\prime \prime}\right)\right\}= \pm 1
$$

The $\vartheta$-function with characteristics $\kappa=\left(\kappa^{\prime} \mid \kappa^{\prime \prime}\right)$ is defined by

$$
\vartheta[\kappa](\zeta, \Omega) \equiv \sum_{n \in \mathbf{Z}^{h}} \exp \left\{\pi i\left(n+\kappa^{\prime}\right) \Omega\left(n+\kappa^{\prime}\right)+2 \pi i\left(n+\kappa^{\prime}\right)\left(\zeta+\kappa^{\prime \prime}\right)\right\}
$$

It satisfies a number of monodromy relations, which are listed in Appendix B. For genus 1 , the relations between the $\vartheta$-functions with characteristics and the standard Jacobi $\vartheta$ functions are as follows. For odd spin structure, we have $\vartheta_{1}(z \mid \tau) \equiv \vartheta\left[\nu_{0}\right](z \mid \tau)$, while 
for even spin structures, we have $\vartheta_{i}(z \mid \tau) \equiv \vartheta\left[\mu_{i}\right](z \mid \tau)$, with the corresponding even spin structure characteristics given by

$$
\mu_{2}=\left(\frac{1}{2} \mid 0\right) \quad \mu_{3}=(0 \mid 0) \quad \mu_{4}=\left(0 \mid \frac{1}{2}\right)
$$

Two fundamental building blocks for the theory of functions and forms on a Riemann surface $^{5}$ are the prime form $E(z, w)$ and the Szegö kernel $S_{\delta}(z, w)$ (which we shall only need for even spin structure $\delta$ ),

$$
E(z, w)=\frac{\vartheta[\nu]\left(\int_{w}^{z} \omega_{I}, \Omega\right)}{h_{\nu}(z) h_{\nu}(w)} \quad S_{\delta}(z, w)=\frac{\vartheta[\delta]\left(\int_{w}^{z} \omega_{I}, \Omega\right)}{\vartheta[\delta](0, \Omega) E(z, w)}
$$

The holomorphic $1 / 2$ form $h_{\nu}(z)$ for odd spin structure $\nu$ obeys $h_{\nu}(z)^{2}=\partial_{I} \vartheta[\nu](0) \omega_{I}(z)$. The prime form is a holomorphic $-\frac{1}{2}$ form in $z$ and $w$ on the universal cover of $\Sigma$, which behaves as $z-w$ when $z \sim w$, and is independent of $\nu$. The Szegö kernel for even spin structure $\delta$ is a meromorphic $\frac{1}{2}$ form in $z$ and $w$ on $\Sigma$ with a simple pole at $z=w$ with unit residue, The importance of these quantities derives from the fact that $S_{\delta}$ is the chiral fermion propagator, while $\partial_{z} \partial_{w} \ln E(z, w)$ is the scalar propagator on $\Sigma$.

\section{$2.2 \quad \mathrm{Z}_{2}$ twisting ; unramified double covers}

The configurations of a scalar $x(z)$ (resp. spinor $\psi(z)$ ) field on $\Sigma$ with a $Z_{2}$-twist correspond to functions (resp. sections of a spin bundle with characteristics $\kappa$ ) that are double-valued on $\Sigma$. These configurations fall into 16 distinct topological sectors corresponding to the elements of $H_{1}\left(\Sigma, \mathbf{Z}_{2}\right)$, and in a given homology basis may be labeled by half characteristics $\varepsilon \equiv\left(\varepsilon^{\prime} \mid \varepsilon^{\prime \prime}\right)$, where $\varepsilon^{\prime}$ and $\varepsilon^{\prime \prime}$ are each valued in $\left\{0, \frac{1}{2}\right\}^{2}$ (see $[27,22]$ ). For the scalar field $x(z)$, and the spinor field $\psi(z)$ with spin structure $\kappa$, we have

$$
\begin{array}{ll}
x\left(z+A_{I}\right)=(-)^{2 \varepsilon_{I}^{\prime}} x(z) & \psi\left(z+A_{I}\right)=-(-)^{2 \varepsilon_{I}^{\prime}+2 \kappa_{I}^{\prime}} \psi(z) \\
x\left(z+B_{I}\right)=(-)^{2 \varepsilon_{I}^{\prime \prime}} x(z) & \psi\left(z+B_{I}\right)=-(-)^{2 \varepsilon_{I}^{\prime \prime}+2 \kappa_{I}^{\prime \prime}} \psi(z)
\end{array}
$$

A $\mathbf{Z}_{2}$-twisted scalar (resp. spinor) field may be viewed as a field defined on the surface $\Sigma$ with a quadratic branch cut along a cycle $C_{\varepsilon}$. The $\mathbf{Z}_{2}$-twisted field is then double-valued around a conjugate cycle $D_{\varepsilon}$, which we parametrize as follows,

$$
D_{\varepsilon}=\sum_{I=1,2}\left(2 \varepsilon_{I}^{\prime} A_{I}+2 \varepsilon_{I}^{\prime \prime} B_{I}\right)
$$

The remaining generators of $H^{1}(\Sigma, \mathbf{Z})$ will be denoted by $A_{\varepsilon}$ and $B_{\varepsilon}$, and have intersection numbers, $\#\left(A_{\varepsilon}, B_{\varepsilon}\right)=\#\left(C_{\varepsilon}, D_{\varepsilon}\right)=1$, while all others vanish. Under the modular group,

\footnotetext{
$[27]$.

${ }^{5}$ For reviews of string perturbation theory, see $[23,25,26]$; for the original mathematical results, see
} 
all twists transform in two orbits, one consisting of $\varepsilon=0$ and the other of all $\varepsilon \neq 0$. It will often be convenient to use the action of the modular group on the twists to choose a standard reference twist. A simple choice consists of taking

$$
\varepsilon=\left(\begin{array}{c|c}
0 & 0 \\
0 & \frac{1}{2}
\end{array}\right) \quad \Leftrightarrow \quad A_{\varepsilon}=A_{1}, B_{\varepsilon}=B_{1}, C_{\varepsilon}=A_{2}, D_{\varepsilon}=B_{2}
$$

It will often be helpful to keep the notation $A_{\varepsilon}, B_{\varepsilon}, C_{\varepsilon}, D_{\varepsilon}$ for the sake of generality.
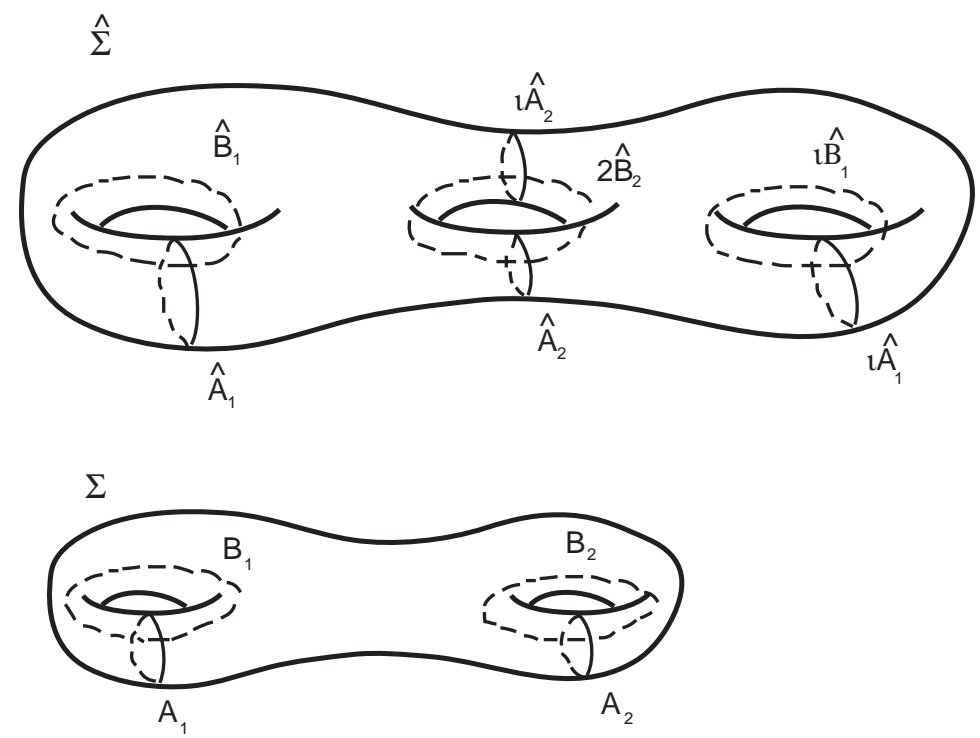

Figure 2: The genus 2 Riemann surface and its unramified branched double cover

Double valued scalar fields on a surface $\Sigma$ may also be viewed as single valued scalar functions of the double cover $\hat{\Sigma}$ of the original surface $\Sigma$. (Similarly, double valued spinor fields may be viewed as well-defined sections of a spin bundle of the cover $\hat{\Sigma}$.) If a function is double valued along the cycle $B_{2}$, and has a quadratic branch cut across the conjugate cycle $A_{2}$, the surface $\hat{\Sigma}$ is obtained by cutting open $\Sigma$ along the cycle $A_{2}$ and gluing the two boundaries of this surface to the boundaries of its image under the reflection involution $\iota$, as shown schematically in Figure 2. Given any twist $\varepsilon \neq 0$, the double cover $\hat{\Sigma}$, as defined above, is unique.

The double cover $\hat{\Sigma}$ is a surface of genus 3, whose complex structure is induced by the complex structure of $\Sigma$. Functions and forms on $\Sigma$ that are $Z_{2}$-twisted (i.e. double-valued) along the cycle $B_{2}=D_{\varepsilon}$, become functions and forms on $\hat{\Sigma}$ that are odd under the reflection involution $\iota$. Of special importance is the Prym differential, $\omega_{\varepsilon}$, which is a holomorphic 1-form, odd under the reflection $\iota$. For genus $2, \omega_{\varepsilon}$ is unique up to normalization. By construction, its periods along the cycles $A_{2}=C_{\varepsilon}$ and $B_{2}=D_{\varepsilon}$ vanish. Normalizing the 
period of $\omega_{\varepsilon}$ to 1 along $A_{1}=A_{\varepsilon}$, its integral along $B_{1}=B_{\varepsilon}$ yields the Prym period $\tau_{\varepsilon}$,

$$
\oint_{A_{\varepsilon}} \omega_{\varepsilon}=1 \quad \oint_{B_{\varepsilon}} \omega_{\varepsilon}=\tau_{\varepsilon}
$$

The Prym period is a complex number satisfying $\operatorname{Im} \tau_{\varepsilon}>0$.

Specification on $\Sigma$ of a twist $\varepsilon \neq 0$, canonically separates the even spin structures $\delta$ into two groups, according to whether $\delta+\varepsilon$ is even or odd. This separation is clearly modular covariant. The group for which $\delta+\varepsilon$ is even consists of 6 elements which will be denoted $\delta_{i}^{+}$and $\delta_{i}^{-}, i=2,3,4$. The group for which $\delta+\varepsilon$ is odd consists of the remaining 4 elements. Without loss of generality, we may assume that $\delta_{i}^{-}=\delta_{i}^{+}+\varepsilon$. For the twist $\varepsilon$ of (2.9), for example, we have

$$
\delta_{i}^{+}=\left(\begin{array}{c}
\mu_{i} \\
0 \mid 0
\end{array}\right) \quad \delta_{i}^{-}=\left(\begin{array}{c}
\mu_{i} \\
0 \mid \frac{1}{2}
\end{array}\right)
$$

where $\mu_{i}$ are the genus 1 even spin structure assignments of (2.5). Clearly, a genus 2 surface with a twist $\varepsilon \neq 0$ has a canonically associated group of 6 even spin structures $\delta_{i}^{ \pm}$, which in turn map 2 to 1 onto the 3 genus 1 even spin structures.

The period matrix $\Omega_{I J}$ of the genus 2 surface uniquely determines the Prym period $\tau_{\varepsilon}$, up to the action of generators in the Torelli group ${ }^{6}$ in this case, up to the transformations $\tau_{\varepsilon} \rightarrow \tau_{\varepsilon}+4$ (see [22]). The relation between $\Omega_{I J}$ and $\tau_{\varepsilon}$ is given by the Schottky relations, already introduced in (1.6). They may be expressed in an equivalent form as follows, for any $i \neq j$ and $i, j=2,3,4$,

$$
\frac{\vartheta\left[\delta_{i}^{+}\right](0, \Omega)^{2} \vartheta\left[\delta_{i}^{-}\right](0, \Omega)^{2}}{\vartheta_{i}\left(0, \tau_{\varepsilon}\right)^{4}}=\frac{\vartheta\left[\delta_{j}^{+}\right](0, \Omega)^{2} \vartheta\left[\delta_{j}^{-}\right](0, \Omega)^{2}}{\vartheta_{j}\left(0, \tau_{\varepsilon}\right)^{4}}
$$

Here, $\delta_{i}^{ \pm}$are the group of 6 even spin structures introduced in the preceding paragraph and $\vartheta_{i}$ are the genus $1 \vartheta$-functions associated with the genus 1 spin structures $\mu_{i}$ onto which $\delta_{i}^{ \pm}$map. Clearly, all three such relations are equivalent to one another. The Schottky relations for genus 2 will be proven and their origin will be explained in section $\S 2.6$.

\subsection{The Dijkgraaf-Verlinde-Verlinde partition function}

The first key result of [22] of interest to us is the formula for the partition function for a single $\mathbf{Z}_{2}$-twisted boson on a circle of radius $R$. In a sector of twist $\varepsilon$, the worldsheet

\footnotetext{
${ }^{6}$ The Torelli group is the quotient of the full mapping class group, consisting of all equivalence classes of disconnected diffeomorphisms of the surface $\Sigma$ by the modular group.
} 
instanton sum is given by

$$
Z[\varepsilon](\Omega, R)=Z^{\mathrm{qu}}[\varepsilon](\Omega) \sum_{\left(p_{L}, p_{R}\right) \in \Gamma_{R}} \exp \left\{\pi i\left(p_{L}^{2} \tau_{\varepsilon}-p_{R}^{2} \bar{\tau}_{\varepsilon}\right)\right\}
$$

where the momentum sum is taken over the lattice

$$
\Gamma_{R}=\left\{\left(p_{L}, p_{R}\right)=\left(\frac{n}{2 R}+m R, \frac{n}{2 R}-m R\right)\right\}
$$

The oscillator part of the partition function is independent of the radius and may be computed by equating the partition functions of the $\mathbf{Z}_{2}$-twisted model with the partition function of the circle theory at the self-dual radius $R=1 / \sqrt{2}$. The result may be expressed in terms of the spin structures $\delta_{i}^{ \pm}$, introduced in the preceding subsection,

$$
Z^{\mathrm{qu}}[\varepsilon](\Omega)=\left|\frac{\vartheta\left[\delta_{i}^{+}\right](0, \Omega) \vartheta\left[\delta_{i}^{-}\right](0, \Omega)}{Z(\Omega)^{2} \vartheta_{i}\left(0, \tau_{\varepsilon}\right)^{2}}\right|
$$

Here, $Z(\Omega)$ is the inverse of the chiral bosonic partition function of the uncompactified circle theory. In view of the Schottky relations (2.12), this expression is independent of the choice of $i$ and therefore only depends upon $\varepsilon$.

\subsection{Genus one $\mathrm{Z}_{2}$-twisted propagators and partition functions}

On the torus $\Sigma=\mathbf{C} / \mathbf{Z}+\tau \mathbf{Z}$, with modulus $\tau$, a scalar field $x(z)$ and a fermion field $\psi(z)$ with even spin structure $\delta$, twisted by $\varepsilon$, obey the following monodromy conditions,

$$
\begin{aligned}
x(z+1)=(-)^{2 \varepsilon^{\prime}} x(z) & \psi(z+1)=-(-)^{2 \delta^{\prime}}(-)^{2 \varepsilon^{\prime}} \psi(z) \\
x(z+\tau)=(-)^{2 \varepsilon^{\prime \prime}} x(z) & \psi(z+\tau)=-(-)^{2 \delta^{\prime \prime}}(-)^{2 \varepsilon^{\prime \prime}} \psi(z)
\end{aligned}
$$

The corresponding propagators satisfy the same monodromy conditions as the fields in both $z$ and $w$. The bosonic propagator $B_{\varepsilon}(z, w)$ is symmetric under interchange of $z$ and $w$, and has a double pole at $z=w$, while the chiral fermion propagator $S_{\delta+\varepsilon}(z, w)$ is antisymmetric under interchange of $z$ and $w$, and has a simple pole at $z=w$. Matching monodromies and poles yields the following propagators,

$$
\begin{aligned}
B_{\varepsilon}(z, w)=\langle\partial x(z) \partial x(w)\rangle & =\partial_{z} \partial_{w} \ln \frac{\vartheta_{1}(z-w \mid 2 \tau)}{\vartheta_{1}(z-w+\tau \mid 2 \tau)} \\
S_{\delta+\varepsilon}(z, w)=\left\langle\psi_{+}(z) \psi_{+}(w)\right\rangle & =\frac{\vartheta[\delta+\varepsilon](z-w, \tau) \vartheta_{1}^{\prime}(0, \tau)}{\vartheta[\delta+\varepsilon](0, \tau) \vartheta_{1}(z-w, \tau)}
\end{aligned}
$$

The chiral fermion propagator is just the Szegö kernel with spin structure shifted to $\delta+\varepsilon$.

It is convenient to recast the twisted boson propagator in terms of the Szegö kernel, since it will turn out that it is these formulas which will lend themselves to generalization 
to higher genus. Using the doubling formulas for genus 1, as well as the formulas for $z$ derivatives (see Appendix A), the following relation is established (valid now for arbitrary twist $\varepsilon \neq 0$ )

$$
B_{\varepsilon}(z, w)=S_{\mu}(z, w) S_{\mu+\epsilon}(z, w)
$$

where $\mu$ is any even spin structure such that $\mu+\varepsilon$ is also even. There are two possible choices for $\mu$ for any given $\varepsilon \neq 0$; e.g. if $\varepsilon=\left(0 \mid \frac{1}{2}\right)$, one can have $\mu=(0 \mid 0)$ or $\mu=\left(0 \mid \frac{1}{2}\right)$.

Theories with worldsheet supersymmetry will be of special interest. The worldsheet supercurrent formed of a free scalar $x$ and a chiral fermion $\psi_{+}$with spin structure $\delta$ is given by $S=-\frac{1}{2} \psi_{+} \partial_{z} x$ and is invariant under the $Z_{2}$ twist $\varepsilon$. The supercurrent two-point function is given by

$$
\langle S(z) S(w)\rangle=\frac{1}{4} B_{\varepsilon}(z, w) S_{\delta+\epsilon}(z, w)=\frac{1}{4} S_{\mu}(z, w) S_{\mu+\epsilon}(z, w) S_{\delta+\epsilon}(z, w)
$$

Using the OPE for the two supercurrents yields the stress tensor for the system,

$$
S(z) S(w)=\frac{\hat{c} / 4}{(z-w)^{3}}+\frac{\frac{1}{2} T(w)}{z-w}+\text { reg }
$$

Expanding the Szegö kernels, one find $\hat{c}=1$, as expected. Using the heat equation, $\partial_{z}^{2} \vartheta[\mu](z \mid \tau)=4 \pi i \partial_{\tau} \vartheta[\mu](z \mid \tau)$, the remaining contribution may be recast as follows,

$$
\langle T\rangle_{\varepsilon, \delta}=\pi i \partial_{\tau} \ln \left(\frac{\vartheta[\mu] \vartheta[\mu+\varepsilon] \vartheta[\delta+\varepsilon]}{\vartheta[0 \mid 0] \vartheta\left[\frac{1}{2} \mid 0\right] \vartheta\left[0 \mid \frac{1}{2}\right]}\right)
$$

The corresponding partition function for one boson and one fermion of spin structure $\delta$, both twisted by $\varepsilon$, are obtained using the relation $\langle T\rangle_{\varepsilon, \delta}=4 \pi / \sqrt{g} \delta \ln Z_{\varepsilon, \delta} / \delta g^{z z}$ and are

$$
Z_{\varepsilon, \delta}=\left(\frac{\vartheta[\delta+\varepsilon](0, \tau)}{\vartheta[\nu+\varepsilon](0, \tau)}\right)^{\frac{1}{2}}
$$

with $\nu=\left(\frac{1}{2} \mid \frac{1}{2}\right)$ in agreement with known results $[28,29]$.

\subsection{Genus two $\mathrm{Z}_{2}$-twisted propagators}

Inspection of the monodromy equations (2.7) reveals that implementing a twist $\varepsilon$ on the fermion field $\psi_{+}(z)$ and on its propagator simply results in replacing the spin structure $\delta$ by $\delta+\varepsilon$. Of interest here are only the cases where $\delta+\varepsilon$ is itself an even spin structure. ${ }^{7}$

\footnotetext{
${ }^{7}$ The cases where $\delta+\varepsilon$ is and odd spin structure will not contribute to the cosmological constant for the orbifold models we shall consider later on.
} 
The propagator for the twisted fermion is thus the Szegö kernel for $\delta+\varepsilon$,

$$
\left\langle\psi_{+}(z) \psi_{+}(w)\right\rangle_{\varepsilon}=S_{\delta+\varepsilon}(z, w)
$$

Viewed on the surface $\Sigma$, this quantity is a double-valued section of a spin bundle for spin structure $\delta$; it becomes a single-valued section of a spin bundle on $\hat{\Sigma}$.

Implementing a twist $\varepsilon$ on a scalar field $x(z)$ is achieved by imposing the monodromy conditions of (2.7). It may be constructed in terms of the prime form for the covering

surface $\hat{\Sigma}$ as was done in [22]. Actually, we shall only need the propagator for the twisted field $\partial_{z} x$,

$$
B_{\varepsilon}(z, w) \equiv\left\langle\partial_{z} x(z) \partial_{w} x(w)\right\rangle_{\varepsilon}
$$

It obeys the same monodromy conditions as $x$ does in both $z$ and $w$ and has a double pole at $z=w$ with unit residue.

By analogy with the case of genus 1 , the propagator $B_{\varepsilon}(z, w)$ may be more simply constructed in terms of products of the Szegö kernels for even spin structures $\delta_{i}^{+}$and $\delta_{i}^{-}$ of (2.11). Indeed, the products

$$
B_{i}(z, w)=S_{\delta_{i}^{+}}(z, w) S_{\delta_{i}^{-}}(z, w) \quad i=2,3,4
$$

readily produce the required double pole. Given the fact that $\delta_{i}^{+}+\delta_{i}^{-}=\varepsilon$ modulo integral periods, they also manifestly exhibit the required monodromy by $\varepsilon$.

The differences $B_{i}(z, w)-B_{j}(z, w)$ are holomorphic 1-forms in both $z$ (as well as in $w$ ), and have the same non-trivial monodromy as $\partial_{z} x$. On a genus 2 surface, such a one-form must be proportional to the Prym differential $\omega_{\varepsilon}(z)$. Since $\omega_{\varepsilon}$ is unique, there must be one linear relation between the three $B_{i}$. Taking this into account, the twisted propagator $B_{\varepsilon}$ may be expressed via any of the following three expressions,

$$
B_{\varepsilon}(z, w)=S_{\delta_{i}^{+}}(z, w) S_{\delta_{i}^{-}}(z, w)+b_{i} \omega_{\varepsilon}(z) \omega_{\varepsilon}(w)
$$

A more detailed discussion of the Prym differential and period will be given later.

\section{The calculation of $b_{i}$}

Below, the coefficient $b_{i}$ will be computed using the partition function for the twisted boson theory, derived in [22] and quoted in $\S 2.3$. The result, in the basis of reference (2.11), is given by

$$
b_{i}=-4 \pi i \partial_{\tau_{\varepsilon}} \ln \vartheta_{i}\left(0, \tau_{\varepsilon}\right)
$$


The detailed calculation of $b_{i}$ proceeds as follows. The stress tensor for the twisted boson $x$ is computed in two different ways; first, from the propagator $B_{\varepsilon}$,

$$
T_{z z}=\frac{1}{2} \lim _{w \rightarrow z}\left(B_{\varepsilon}(z, w)-\frac{1}{(z-w)^{2}}\right)
$$

and second from the variation of the partition function $Z^{\mathrm{qu}}[\varepsilon]$ of the twisted $x$ field,

$$
T_{z z}=\delta_{z z} \ln Z^{\mathrm{qu}}[\varepsilon] \quad \delta_{z z} \equiv \frac{4 \pi}{\sqrt{g}} \frac{\delta}{\delta g^{z z}}
$$

The partition function $Z^{\mathrm{qu}}[\varepsilon]$, was computed in[22], and was presented in the notation of (2.11) in (2.15). Equating (2.28) and (2.29) yields the following results. In the variation (2.29) of (2.15), the variation of $Z$ and of the $\vartheta$-functions $\vartheta\left[\delta_{i}^{+}\right] \vartheta\left[\delta_{i}^{-}\right]$precisely matches the contribution to $(2.28)$ of the first term in (2.26). The remaining variation gives

$$
\frac{1}{2} b_{i} \omega_{\varepsilon}(z)^{2}=-\delta_{z z} \ln \vartheta_{i}\left(0, \tau_{\varepsilon}\right)=-\left(\delta_{z z} \tau_{\varepsilon}\right) \partial \ln \vartheta_{i}\left(0, \tau_{\varepsilon}\right)
$$

The variation of $\tau_{\varepsilon}$ with respect to the metric is analogous to the variation of the genus 2 period matrix, $\delta_{z z} \Omega_{I J}=2 \pi i \omega_{I}(z) \omega_{J}(z)$. In the next subsection (where a detailed construction of the Prym differential will also be given), we shall derive the following expression,

$$
\delta_{z z} \tau_{\varepsilon}=2 \pi i \omega_{\varepsilon}(z)^{2}
$$

Combining these results readily yields (2.27).

\subsection{The Prym differential and the Schottky relations}

The construction of the normalized Prym differential, the Schottky relations and the variational equation (2.31) for $\tau_{\varepsilon}$ require a more concrete understanding of the Prym differential. It is convenient to obtain the Prym differential from its relation to the Szegö kernel,

$$
\omega_{\varepsilon}(z) \omega_{\varepsilon}(w) \sim\left(S_{\delta_{i}^{+}}(z, w) S_{\delta_{i}^{-}}(z, w)-S_{\delta_{j}^{+}}(z, w) S_{\delta_{j}^{-}}(z, w)\right)
$$

The constants of proportionality in this relation will be determined below.

To do so, the hyperelliptic parametrization of genus 2 Riemann surfaces is used ${ }^{8}$. The branch points are denoted by $p_{1}, \cdots, p_{6}$ and the corresponding hyperelliptic curve is

$$
s^{2}=\prod_{a=1}^{6}\left(z-p_{a}\right)
$$

\footnotetext{
${ }^{8} \mathrm{~A}$ detailed discussion of the map between the $\vartheta$-function and hyperelliptic formulations of genus 2 Riemann surfaces was given in [4]; see Appendix B of this paper for a summary of the relevant facts.
} 
Recall that the identification between even and odd spin structures and branch points proceeds as follows,

$$
\begin{aligned}
& \nu_{a} \text { odd } \leftrightarrow \text { branch point } p_{a} \\
& \delta \quad \text { even } \leftrightarrow \text { partition } A \cup B \quad A=\left\{p_{a_{1}}, p_{a_{2}}, p_{a_{3}}\right\}, \quad B=\left\{p_{b_{1}}, p_{b_{2}}, p_{b_{3}}\right\}
\end{aligned}
$$

The twist will be left general. To this end, spin structures and twists will be parametrized by odd spin structures, $\left\{\nu_{a}, \nu_{b}, \nu_{c}, \nu_{d}, \nu_{e}, \nu_{f}\right\}$, where (abcdef) is a permutation of (123456). The ${ }_{6} C_{2}=15(=16-1)$ non-zero twists $\varepsilon$ can be uniquely labeled by two distinct odd spin structures $\nu_{a, b}$, such that $\varepsilon=\nu_{a}+\nu_{b} \not \equiv 0$. The same twist can also be characterized as a sum of two even spin structures, $\varepsilon=-\delta_{i}^{+}+\delta_{i}^{-}$. There are 3 distinct choices for these sets which we label here as

$$
\begin{aligned}
& \delta_{i}^{+}=\nu_{a}+\nu_{c}+\nu_{d}=-\left(\nu_{b}+\nu_{e}+\nu_{f}\right) \\
& \delta_{i}^{-}=\nu_{b}+\nu_{c}+\nu_{d}=-\left(\nu_{a}+\nu_{e}+\nu_{f}\right) \\
& \delta_{j}^{+}=\nu_{a}+\nu_{d}+\nu_{e}=-\left(\nu_{b}+\nu_{c}+\nu_{f}\right) \\
& \delta_{j}^{-}=\nu_{b}+\nu_{d}+\nu_{e}=-\left(\nu_{a}+\nu_{c}+\nu_{f}\right) \\
& \delta_{k}^{+}=\nu_{a}+\nu_{c}+\nu_{e}=-\left(\nu_{b}+\nu_{d}+\nu_{f}\right) \\
& \delta_{k}^{-}=\nu_{b}+\nu_{c}+\nu_{e}=-\left(\nu_{a}+\nu_{d}+\nu_{f}\right)
\end{aligned}
$$

We note that $a, b$ cannot belong to the same set in the partition associated with the spin structure $\delta$ since then, $\delta+\varepsilon$ would be odd.

Let $\delta$ be the even spin structure corresponding to the partition of the 6 branch points into two sets $A, B$ of 3 branch points each, as in (B.18). In the hyperelliptic representation, the Szegö kernel for the spin structure $\delta$ is given by [27],

$$
S_{\delta}(z, w)=\frac{1}{2} \frac{s_{A}(z) s_{B}(w)+s_{A}(w) s_{B}(z)}{z-w}\left(\frac{d z}{s(z)}\right)^{\frac{1}{2}}\left(\frac{d w}{s(w)}\right)^{\frac{1}{2}}
$$

where the following notation is used,

$$
\begin{aligned}
s_{A}(z)^{2} & =\left(z-p_{a_{1}}\right)\left(z-p_{a_{2}}\right)\left(z-p_{a_{3}}\right) \\
s_{B}(z)^{2} & =\left(z-p_{b_{1}}\right)\left(z-p_{b_{2}}\right)\left(z-p_{b_{3}}\right) \\
s(z)^{2} & =\left(z-p_{1}\right)\left(z-p_{2}\right)\left(z-p_{3}\right)\left(z-p_{4}\right)\left(z-p_{5}\right)\left(z-p_{6}\right)
\end{aligned}
$$

The combinations of Szegö kernels that enter into (2.32) may be expressed in this hyperelliptic formulation,

$$
\begin{aligned}
S_{\delta_{j}^{+}}(z, w) S_{\delta_{j}^{-}}(z, w)-S_{\delta_{k}^{+}}(z, w) S_{\delta_{k}^{-}}(z, w) & =\frac{\left(p_{c}-p_{d}\right)\left(p_{f}-p_{e}\right)}{4 s_{\text {cdef }}(z) s_{c d e f}(w)} d z d w \\
S_{\delta_{k}^{+}}(z, w) S_{\delta_{k}^{-}}(z, w)-S_{\delta_{i}^{+}}(z, w) S_{\delta_{i}^{-}}(z, w) & =\frac{\left(p_{c}-p_{f}\right)\left(p_{e}-p_{d}\right)}{4 s_{\text {cdef }}(z) s_{c d e f}(w)} d z d w \\
S_{\delta_{i}^{+}}(z, w) S_{\delta_{i}^{-}}(z, w)-S_{\delta_{j}^{+}}(z, w) S_{\delta_{j}^{-}}(z, w) & =\frac{\left(p_{c}-p_{e}\right)\left(p_{d}-p_{f}\right)}{4 s_{c d e f}(z) s_{c d e f}(w)} d z d w
\end{aligned}
$$


The hyperelliptic form of the Prym differential is thus given by

$$
\omega_{\varepsilon}(z) \sim \frac{d z}{s_{c d e f}(z)}
$$

This quantity involves only 4 branch points, and by $S L(2, \mathbf{C})$-invariance depends on a single modulus, which is the Prym period $\tau_{\varepsilon}$.

\section{The Schottky relations}

The Schottky relations arise from relating the modulus of the genus 1 curve with branch points $p_{c}, p_{d}, p_{e}, p_{f}$ to the moduli of the full genus 2 curve with all 6 branch points. Without loss of generality, the following identification $\left(e_{j}, e_{k}, e_{i}, \infty\right)=\left(p_{c}, p_{d}, p_{e}, p_{f}\right)$ can be made, which allows us to put the elliptic curve in standard from,

$$
y^{2}=4\left(x-e_{2}\right)\left(x-e_{3}\right)\left(x-e_{4}\right)=4\left(x-p_{c}\right)\left(x-p_{d}\right)\left(x-p_{e}\right)
$$

The genus 1 Thomae formulas then yield, (see [30], page 361, equations (7))

$$
\begin{aligned}
e_{j}-e_{k} & =p_{c}-p_{d}=\sigma\left(\mu_{j}, \mu_{k}\right) \frac{\pi^{2}}{4 \omega^{2}} \vartheta_{i}^{4} \\
e_{k}-e_{i} & =p_{d}-p_{e}=\sigma\left(\mu_{k}, \mu_{i}\right) \frac{\pi^{2}}{4 \omega^{2}} \vartheta_{j}^{4} \\
e_{i}-e_{j} & =p_{e}-p_{c}=\sigma\left(\mu_{i}, \mu_{j}\right) \frac{\pi^{2}}{4 \omega^{2}} \vartheta_{k}^{4}
\end{aligned}
$$

The function $\sigma$ is anti-symmetric, $\sigma\left(\mu_{i}, \mu_{j}\right)=-\sigma\left(\mu_{j}, \mu_{i}\right)$ and is normalized by

$$
\sigma\left(\mu_{2}, \mu_{3}\right)=\sigma\left(\mu_{3}, \mu_{4}\right)=\sigma\left(\mu_{2}, \mu_{4}\right)=+1
$$

The consistency of these relations follows from the genus 1 Jacobi identity,

$$
\sigma\left(\mu_{i}, \mu_{j}\right) \vartheta_{k}^{4}+\sigma\left(\mu_{j}, \mu_{k}\right) \vartheta_{i}^{4}+\sigma\left(\mu_{k}, \mu_{i}\right) \vartheta_{j}^{4}=0
$$

Furthermore, $2 \omega$ is the $A_{\varepsilon}$ period of the elliptic curve. Recall from [4] that, in terms of the modular object $\mathcal{M}_{\nu_{a} \nu_{b}}$,

$$
\begin{aligned}
\mathcal{M}_{\nu_{a} \nu_{b}} & \equiv \partial_{1} \vartheta\left[\nu_{a}\right](0, \Omega) \partial_{2} \vartheta\left[\nu_{b}\right](0, \Omega)-\partial_{2} \vartheta\left[\nu_{a}\right](0, \Omega) \partial_{1} \vartheta\left[\nu_{b}\right](0, \Omega) \\
\mathcal{M}_{\nu_{a} \nu_{b}}^{2} & =\pi^{4} \prod_{k \neq a, b}^{6} \vartheta\left[\nu_{a}+\nu_{b}+\nu_{k}\right](0, \Omega)^{2}
\end{aligned}
$$

we have a refined form of the genus 2 Thomae identities,

$$
\frac{\left(p_{a}-p_{b}\right)\left(p_{c}-p_{d}\right)}{\left(p_{a}-p_{c}\right)\left(p_{b}-p_{d}\right)}=\frac{\mathcal{M}_{\nu_{a} \nu_{b}} \mathcal{M}_{\nu_{c} \nu_{d}}}{\mathcal{M}_{\nu_{a} \nu_{c}} \mathcal{M}_{\nu_{b} \nu_{d}}}
$$


The identification leads to the following relations between the three cross ratios that may be constructed out of the 4 points $c, d, e, f$,

$$
\frac{\vartheta_{i}^{8}}{\vartheta_{j}^{8}}\left(0, \tau_{\varepsilon}\right)=\left(\frac{e_{j}-e_{k}}{e_{k}-e_{i}}\right)^{2}=\frac{\mathcal{M}_{c d}^{2} \mathcal{M}_{e f}^{2}}{\mathcal{M}_{c f}^{2} \mathcal{M}_{d e}^{2}}=\frac{\vartheta^{4}\left[\delta_{i}^{+}\right] \vartheta^{4}\left[\delta_{i}^{-}\right]}{\vartheta^{4}\left[\delta_{j}^{+}\right] \vartheta^{4}\left[\delta_{j}^{-}\right]}(0, \Omega)
$$

In particular, the relation between the separating degeneration limit of the genus 2 periods and the Prym period is $\vartheta\left[\mu_{i}\right]\left(0, \Omega_{11}\right)^{8}=\vartheta\left[\mu_{i}\right]\left(0, \tau_{\varepsilon}\right)^{8}$ so that $\tau_{\varepsilon}=\Omega_{11}+\mathcal{O}\left(\Omega_{12}\right)$ up to the possible addition of an integer multiple of 8 .

\section{The Prym differential in terms of Szego kernels}

It is now straightforward to construct the normalized Prym differential. Still in the conventions of [30] (pages 330 and 331), the $A_{\varepsilon}$ cycle is chosen so that it produces the period $2 \omega$. This is achieved by taking $A_{\varepsilon}$ to be the cycle around the branch cut between the branch points $e_{k}$ and $e_{i}$, while the $B_{\varepsilon}$ cycle goes from $e_{j}$ to $e_{k}$,

$$
\omega=\int_{e_{k}}^{e_{i}} \frac{d x}{y(x)} \quad \omega^{\prime}=\int_{e_{j}}^{e_{k}} \frac{d x}{y(x)}
$$

Therefore, the normalized Prym differential is simply

$$
\omega_{\varepsilon}(x) \equiv \frac{1}{2 \omega} \frac{d x}{y(x)}
$$

With this normalization, the relations between the normalized Prym differential and the Szegö kernel are completely fixed and are found to be

$$
S_{\delta_{i}^{+}}(z, w) S_{\delta_{i}^{-}}(z, w)-S_{\delta_{j}^{+}}(z, w) S_{\delta_{j}^{-}}(z, w)=-\sigma\left(\mu_{i}, \mu_{j}\right) \pi^{2} \vartheta_{k}\left(0, \tau_{\varepsilon}\right)^{4} \omega_{\varepsilon}(z) \omega_{\varepsilon}(w)
$$

Clearly, the sum of the left terms vanishes and so does the sum of the right terms in view of the genus 1 Jacobi identity (2.43).

The square of the Prym differential may be deduced from the expression in terms of the product of Szegö kernels, evaluated as $w \rightarrow z$,

$$
\begin{aligned}
\omega_{\varepsilon}(z)^{2} & =-\sigma\left(\mu_{i}, \mu_{j}\right) \frac{1}{\pi^{2} \vartheta_{k}^{4}} \lim _{w \rightarrow z}\left(S_{\delta_{i}^{+}}(z, w) S_{\delta_{i}^{-}}(z, w)-S_{\delta_{j}^{+}}(z, w) S_{\delta_{j}^{-}}(z, w)\right) \\
& =-\sigma\left(\mu_{i}, \mu_{j}\right) \frac{1}{2 \pi^{2} \vartheta_{k}^{4}} \sum_{I, J} \omega_{I}(z) \omega_{J}(z) \partial_{I} \partial_{J} \ln \left(\frac{\vartheta\left[\delta_{i}^{+}\right] \vartheta\left[\delta_{i}^{-}\right]}{\vartheta\left[\delta_{j}^{+}\right] \vartheta\left[\delta_{j}^{-}\right]}\right)
\end{aligned}
$$

Differentiating relation $(2.46)$, with respect to $\Omega_{I J}$, we have

$$
\left(\partial_{I J} \tau_{\varepsilon}\right) \partial_{\tau_{\varepsilon}} \ln \frac{\vartheta_{i}}{\vartheta_{j}}\left(0, \tau_{\varepsilon}\right)=\frac{1}{2} \partial_{I J} \ln \left(\frac{\vartheta\left[\delta_{i}^{+}\right] \vartheta\left[\delta_{i}^{-}\right]}{\vartheta\left[\delta_{j}^{+}\right] \vartheta\left[\delta_{j}^{-}\right]}\right)
$$

Using the heat equation $4 \pi i \partial_{I J} \vartheta=\partial_{I} \partial_{J} \vartheta$, the relation $\delta_{z z} \Omega_{I J}=2 \pi i \omega_{I}(z) \omega_{J}(z)$ and

$$
\partial_{\tau_{\varepsilon}} \ln \frac{\vartheta_{i}}{\vartheta_{j}}\left(0, \tau_{\varepsilon}\right)=i \frac{\pi}{4} \sigma\left(\mu_{i}, \mu_{j}\right) \vartheta_{k}\left(0, \tau_{\varepsilon}\right)^{4}
$$

yields the equation announced in (2.31). 


\section{Chiral Splitting for $\mathrm{Z}_{2}$ Orbifold Theories}

Chiral splitting is the process of decomposition of a chirally symmetric amplitude into a sum of terms, each of which may be written as the absolute value square of a chiral block which is meromorphic on supermoduli space and has meromorphic dependence on the vertex insertion data $[20,21]$. Each chiral block corresponds to a single $\mathcal{N}=1$ superconformal family. The chiral blocks thus obtained may be used to carry out chiral GSO projections [31], which are required for the construction of both the Type II [32] and Heterotic [33] superstring theories. The precise manner in which chirally symmetric amplitudes are chirally split was worked out long ago for flat space-time [21] (see also [23] and [20]). In the present section, chiral splitting will be carried out in detail in the $\mathbf{Z}_{2}$-twisted sectors for orbifolds of flat space-time involving $\mathbf{Z}_{2}$ twistings. For definiteness, the restriction to two-loops is made from the outset.

\subsection{Chirally Symmetric Amplitudes}

The compactified space-time $C$ will be viewed as an $\mathcal{N}=1$ super conformal field theory on a worldsheet $\Sigma$ of genus 2, coupled to a two-dimensional supergeometry with superframe $E_{M}{ }^{A}$ and $U(1)$ superconnection $\Omega_{M},[34,23]$. The supergeometry $\left(E_{M}{ }^{A}, \Omega_{M}\right)$ is subject to the Wess-Zumino constraints, indicated here by the delta function $\delta(T)$. In Wess-Zumino gauge, we have $E_{m}{ }^{a}=e_{m}{ }^{a}+\theta \gamma^{a} \chi_{m}$, omitting the auxiliary field, $e_{m}{ }^{a}$ is a frame for the worldsheet metric $g_{m n}=e_{m}{ }^{a} e_{n}{ }^{b} \delta_{a b}$, and $\chi_{m}{ }^{\alpha}=\left(\chi_{\bar{z}}^{+}, \chi_{z}{ }^{-}\right)$is the worldsheet gravitino field. The definition of a supergeometry requires a spin structure $\delta$ on the worldsheet.

For fixed spin structure $\delta$, superstring scattering amplitudes are built out of chirally symmetric correlations functions of $C$ coupled to two-dimensional supergeometry,

$$
\mathbf{A}_{C}[\delta]=\int D E_{M}^{A} D \Omega_{M} \delta(T) \int_{C} D X^{\mu}\left(\prod_{i=1}^{N} V_{i}\right) e^{-I_{m}}
$$

Here, $V_{i}$ are vertex operators for physical states and the subscript $C$ stands for the functional integral evaluated in the superconformal field theory associated with $C$. Upon factoring out the local symmetries of the theory, the integration over supergeometries $\left(E_{M}{ }^{A}, \Omega_{M}\right)$ reduces to an integral over supermoduli space [35, 20], which is defined by

$$
s \mathcal{M}_{2}=\left\{\left(E_{M}^{A}, \Omega_{M}\right)\right\} /\{\text { Gauge Symmetries }\}
$$

This integral can be written explicitly by choosing a (3|2)-dimensional slice $\mathcal{S}$ of supergeometries $\left(E_{M}^{A}, \Omega_{M}\right)([2]$, eq. (2.11)),

$$
\mathbf{A}_{C}[\delta]=\int_{s \mathcal{M}_{2}} \prod_{A}\left|d m^{A}\right|^{2} \int D(X B \bar{B} C \bar{C}) \prod_{A}\left|\delta\left(\left\langle H_{A} \mid B\right\rangle\right)\right|^{2}\left(\prod_{i=1}^{N} V_{i}\right) e^{-I_{m}-I_{g h}}
$$

In this formula, $m^{A}=\left(m^{a}, \zeta^{\alpha}\right)$ is a set of local complex coordinates on $s \mathcal{M}_{2}$ where $A$ runs over the $(3 \mid 2)$ complex dimensions of $s \mathcal{M}_{2}$ and $H_{A}$ are the Beltrami superdifferentials for 
the slice chosen to represent $s \mathcal{M}_{2}$. The superfields $B$ and $C$ represent all the superghosts; in standard component notation, they are given by $B=\beta+\theta b$ and $C=c+\theta \gamma$, omitting their corresponding auxiliary fields.

For orbifold compactifications, the starting point will be the matter action for flat space-time. The matter superfield has the following component decomposition, $X^{\mu}=$ $x^{\mu}+\theta \psi_{+}^{\mu}+\bar{\theta} \psi_{-}^{\mu}$, up to auxiliary fields. Henceforth, we restrict to the case of $\mathbf{Z}_{2}$-orbifolds and we have,

$$
\begin{aligned}
I_{m}= & \frac{1}{4 \pi} \int d^{2 \mid 2} z\left(\operatorname{sdet} E_{M}{ }^{A}\right) \mathcal{D}_{+} X^{\mu} \mathcal{D}_{-} X^{\mu} \\
= & \frac{1}{4 \pi} \int d^{2} z\left(\partial_{z} x^{\mu} \partial_{\bar{z}} x^{\mu}-\psi_{+}^{\mu} \partial_{\bar{z}} \psi_{+}^{\mu}-\psi_{-}^{\mu} \partial_{z} \psi_{-}^{\mu}\right. \\
& \left.\quad-\chi_{\bar{z}}^{+} S_{m}-\chi_{z}^{-} \overline{S_{m}}-\frac{1}{4} \chi_{\bar{z}}^{+} \chi_{z}^{-} \psi_{+}^{\mu} \psi_{-}^{\mu}\right)
\end{aligned}
$$

while the ghost action is given by

$$
\begin{aligned}
I_{g h} & =\frac{1}{2 \pi} \int d^{2 \mid 2} z\left(\operatorname{sdet} E_{M}{ }^{A}\right)\left(B \mathcal{D}_{-} C+\bar{B} \mathcal{D}_{+} \bar{C}\right) \\
& =\frac{1}{2 \pi} \int d^{2} z\left(b \partial_{\bar{z}} c+\beta \partial_{\bar{z}} \gamma+\bar{b} \partial_{z} \bar{c}+\bar{\beta} \partial_{\bar{z}} \bar{\gamma}-\chi_{\bar{z}}^{+} S_{g h}-\chi_{z}^{-} \overline{S_{g h}}\right)
\end{aligned}
$$

The matter and ghost supercurrents are given by

$$
\begin{aligned}
S_{m} & =-\frac{1}{2} \psi_{+}^{\mu} \partial_{z} x_{+}^{\mu} \\
S_{g h} & =\frac{1}{2} b \gamma-\frac{3}{2} \beta \partial_{z} c-\left(\partial_{z} \beta\right) c
\end{aligned}
$$

These formulas are identical in form to those for flat space-time, with the exception that here, both the boson $x^{\mu}$ and fermion $\psi_{ \pm}^{\mu}$ are $\mathbf{Z}_{2}$-twisted by the same twist $\varepsilon$. This guarantees in particular that the matter supercurrent and stress tensor are single-valued.

\subsection{Chiral splitting of general amplitudes in $\mathrm{Z}_{2}$-twisted sectors}

Let $\varepsilon \neq 0$ denote the $\mathbf{Z}_{2}$-twist which is applied to $n$ components of the scalar field $x$ and $n$ components of the fermion fields $\psi_{ \pm}$taken to have spin structure $\delta$. This means that these fields satisfy the twisted boundary conditions of (2.7). The resulting chiral scalar and chiral fermion propagators were computed in $\S 2$, and are given by

$$
\begin{aligned}
\left\langle\partial_{z} x_{+}(z) \partial_{w} x_{+}(w)\right\rangle_{\varepsilon} & =B_{\varepsilon}(z, w) \\
\left\langle\psi_{+}(z) \psi_{+}(w)\right\rangle_{\varepsilon} & =S_{\delta+\varepsilon}(z, w)
\end{aligned}
$$

The functional integral over $n$ components of twisted $\psi_{ \pm}$fields is given by the determinant of the Dirac determinant, which is holomorphically factorized (up to the Belavin-Knizhnik 
anomaly [36], whose effects cancel in the full amplitude),

$$
(\operatorname{Det} \not D)^{\frac{n}{2}}=\left|\frac{\vartheta[\delta+\varepsilon](0, \Omega)}{Z(\Omega)}\right|^{n}
$$

The quantity $Z$ is the inverse of the chiral partition function of a free untwisted scalar; its expression in terms of $\vartheta$-functions was given in [2], eq. (4.5) but will not be needed here. The functional integral over $x^{\mu}$ in the twisted sector is simpler than in the untwisted sector, because no zero-mode occurs in the twisted sectors. By the Belavin-Knizhnik result [36], the combination of determinants that holomorphically factorizes involves the inner products of holomorphic one forms. In the twisted sector there is precisely one such form, namely the Prym differential $\omega_{\varepsilon}$, normalized as in (2.10). The functional determinant over $n$ twisted fields $x$ is then given by the following expression,

$$
(\operatorname{Det} \Delta)^{-\frac{n}{2}}=\frac{1}{\left(2 \operatorname{Im} \tau_{\varepsilon}\right)^{\frac{n}{2}}}\left|\frac{\vartheta\left[\delta_{j}^{+}\right](0, \Omega) \vartheta\left[\delta_{j}^{-}\right](0, \Omega)}{Z(\Omega)^{2} \vartheta_{j}(0, \tau)^{2}}\right|^{n}
$$

The obstruction to its holomorphic factorization lies entirely in the prefactor involving $\operatorname{Im} \tau_{\varepsilon}$. Chiral splitting is achieved by introducing a single internal loop momentum $p_{\varepsilon}$, in terms of which also the prefactor may be split,

$$
\frac{1}{\left(2 \operatorname{Im} \tau_{\varepsilon}\right)^{\frac{n}{2}}}=\int d^{n} p_{\varepsilon}\left|e^{i \pi \tau_{\varepsilon} p_{\varepsilon}^{2}}\right|^{2}
$$

The functional integral (3.3) exhibits further obstructions to chiral splitting. The first is through the presence of the quartic fermion term $\chi \bar{\chi} \psi_{+} \psi_{-}$in the matter action $I_{m}$; the second is through the non-holomorphic dependence of the full fermion propagator,

$$
\begin{aligned}
\left\langle\partial_{z} x(z) \partial_{w} x(w)\right\rangle_{\varepsilon} & =B_{\varepsilon}(z, w)-\frac{2 \pi}{\operatorname{Im} \tau_{\varepsilon}} \omega_{\varepsilon}(z) \omega_{\varepsilon}(w) \\
\left\langle\partial_{z} x(z) \partial_{\bar{w}} x(w)\right\rangle_{\varepsilon} & =-2 \pi \delta(z, w)+\frac{2 \pi}{\operatorname{Im} \tau_{\varepsilon}} \omega_{\varepsilon}(z) \overline{\omega_{\varepsilon}(w)}
\end{aligned}
$$

The first line may be established by comparing the stress tensors computed from a variation of the scalar determinant (3.9) and from the full $x$-field propagator. This procedure is the same as the one used in (2.28) and (2.29), but is now applied to the full twisted scalar partition function and propagator. The second line in (3.11) is obtained by applying $\partial_{\bar{w}}$ to the first line and then integrating in $w$; the remaining coefficient in front of $\omega_{\varepsilon}(z) \omega_{\varepsilon}(w)$ is fixed by requiring that the integration versus $\omega_{\varepsilon}(w)$ vanish.

Next, one proceeds in parallel with the proof of chiral splitting for the case of flat space-time $[23,21]$. The result may be summarized in terms of a set of effective rules. The 
final formula for the integration over the matter fields may be recast in the following form,

$$
\begin{aligned}
& \mathbf{A}_{C}[\delta]=\sum_{\varepsilon} \int d^{10-n} p_{I} \int d^{n} p_{\varepsilon} \int_{s \mathcal{M}_{2}} \prod_{A}\left|d m^{A}\right|^{2}\left|\mathcal{A}_{C}[\delta, \varepsilon]\left(p_{\varepsilon}\right)\right|^{2} \mid e^{\left.i \pi p_{I}^{\mu} \hat{\Omega}_{I J} p_{J}^{\mu}\right|^{2}} \\
& \mathcal{A}_{C}[\delta, \varepsilon]\left(p_{\varepsilon}\right)=\left\langle\prod_{A} \delta\left(\left\langle H_{A} \mid B\right\rangle\right) \prod_{i=1}^{N} V_{i}^{\operatorname{chi}} \exp \left\{\int \frac{d^{2} z}{2 \pi} \chi_{\bar{z}}^{+} S(z)+p_{\varepsilon}^{\mu} \oint_{B_{\varepsilon}} d z \partial_{z} x_{+}^{\mu}\right\}\right\rangle_{+}
\end{aligned}
$$

Here, $S(z)$ is the total worldsheet supercurrent, given by $S(z)=S_{m}(z)+S_{g h}(z)$. The variance of the Gaussian in the first line is given by the super-period matrix, which is a shift of the bosonic period matrix by an even, Grassmann valued, and nilpotent element, given by [23],

$$
\hat{\Omega}_{I J}=\Omega_{I J}-\frac{i}{8 \pi} \int d^{2} z \int d^{2} w \omega_{I}(z) \chi_{\bar{z}}^{+} S_{\delta}(z, w) \chi_{\bar{w}}^{+} \omega_{J}(w)
$$

Furthermore, $V_{i}^{\text {chi }}$ is the chiral part of the vertex operator $V_{i}$. All contractions in this chiral correlator $\langle\cdots\rangle_{+}$are to be carried out with the help of the propagators for the chiral fields $x_{+}$and $\psi_{+}$given in (3.7). In the present paper, the emphasis will be on the chiral measure and the cosmological constant. Therefore, the precise form of the operators $V_{i}$ and their chiral part $V_{i}^{\text {chi }}$ will not be needed and will not be presented here.

\subsection{The chiral measure in the $\mathrm{Z}_{2}$-twisted sectors}

In the absence of vertex operator insertions, the $p_{\varepsilon}^{\mu}$-dependence of the amplitude arises from the $x_{+}^{\mu}$-contractions of the term involving $p_{\varepsilon}^{\mu}$ with itself and with $S_{m}$, yielding

$$
\begin{aligned}
\left\langle\exp \left\{\int \frac{d^{2} z}{2 \pi} \chi_{\bar{z}}^{+} S(z)+p_{\varepsilon}^{\mu} \oint_{B_{\varepsilon}} d z \partial_{z} x_{+}^{\mu}\right\}\right\rangle_{x_{+}, \psi_{+}} & \\
= & \left\langle\operatorname { e x p } \left\{ i \pi \tau_{\varepsilon} p_{\varepsilon}^{2}-\frac{1}{2} p_{\varepsilon}^{\mu} \int d^{2} z \chi_{\bar{z}}^{+} \psi_{+}^{\mu} \omega_{\varepsilon}(z)\right.\right. \\
& \left.\quad-\frac{1}{8 \pi} \int d^{2} z \int d^{2} w \chi_{\bar{z}}^{+} \chi_{\bar{w}}^{+}\langle S(z) S(w)\rangle_{x_{+}}\right\rangle_{\psi_{+}}\langle 1\rangle_{x_{+}}
\end{aligned}
$$

where $\langle 1\rangle_{x_{+}}$denotes the $\mathbf{Z}_{2}$-twisted chiral boson partition function. Carrying out also the $\psi_{+}$contractions of the $p_{\varepsilon}$-dependent terms, the following result is obtained,

$$
\begin{aligned}
\langle\exp & \left.\left\{\int \frac{d^{2} z}{2 \pi} \chi_{\bar{z}}^{+} S(z)+p_{\varepsilon}^{\mu} \oint_{B_{\varepsilon}} d z \partial_{z} x_{+}^{\mu}\right\}\right\rangle_{x_{+}, \psi_{+}} \\
& =\left(1-\frac{1}{8 \pi^{2}} \int d^{2} z \int d^{2} w \chi_{\bar{z}}^{+} \chi_{\bar{w}}^{+}\langle S(z) S(w)\rangle_{x_{+}}\right) Z_{C}[\delta ; \varepsilon] \exp \left\{i \pi \tilde{\tau}_{\varepsilon} p_{\varepsilon}^{2}\right\}
\end{aligned}
$$

Here, the product of the $\mathbf{Z}_{2}$-twisted chiral scalar and fermion partition functions $\langle 1\rangle_{x_{+}}$and $\langle 1\rangle_{\psi_{+}}$is denoted by $Z_{C}[\delta, \varepsilon]$. In the above formula, all the $p_{\varepsilon}$-dependence may be regrouped 
in terms of a Gaussian with the following variance,

$$
\tilde{\tau} \equiv \tau-\frac{i}{8 \pi} \int d^{2} z \int d^{2} w \chi_{\bar{z}}^{+} \omega_{\varepsilon}(z) S_{\delta+\varepsilon}(z, w) \chi_{\bar{w}}^{+} \omega_{\varepsilon}(w)
$$

This correction has the same origin as the corrections to the period matrix that lead to the super period matrix in the uncompactified string. Its proper interpretation here is, however, more subtle, and will be presented in detail later.

The contributions of the ghost partition function and supercurrent correlators are the same as they were in flat space-time and may be taken from [2]. Assembling all contributions, the chiral measure is given by the following expression

$$
\begin{aligned}
\mathcal{A}_{C}[\delta ; \varepsilon]\left(p_{\varepsilon}\right)=\frac{\left\langle\prod_{a} b\left(p_{a}\right) \prod_{\alpha} \delta\left(\beta\left(q_{\alpha}\right)\right)\right\rangle_{M}}{\operatorname{det} \Phi_{I J+}\left(p_{a}\right) \cdot \operatorname{det}\left\langle H_{\alpha} \mid \Phi_{\beta}^{*}\right\rangle} \frac{Z_{C}[\delta ; \varepsilon]}{Z_{M}[\delta]} \exp \left\{i \pi \tilde{\tau}_{\varepsilon} p_{\varepsilon}^{2}\right\} \\
\quad \times\left\{1-\frac{1}{8 \pi^{2}} \int d^{2} z \int d^{2} w \chi_{\bar{z}}^{+} \chi_{\bar{w}}^{+}\langle S(z) S(w)\rangle_{C}\right\}
\end{aligned}
$$

The factor of $Z_{M}[\delta]$ stands for the chiral matter partition function of flat space-time; it is of course independent of the twist $\varepsilon$. This factor must be divided out since it was already included in the definition used in [2] for the matter-ghost correlator $\left\langle\prod_{a} b\left(p_{a}\right) \prod_{\alpha} \delta\left(\beta\left(q_{\alpha}\right)\right)\right\rangle_{M}$ in flat space-time. Detailed definitions and explicit expressions for the various ingredients in the above formula were given in [2]. Suffice it here to remind the reader that the ghost insertion points $p_{a}$ and $q_{\alpha}$, with $a=1,2,3$ and $\alpha=1,2$ are arbitrary; that $\Phi_{I J}$ and $\Phi_{B}^{*}$ are superholomorphic $3 / 2$ forms and $H_{A}$ is a super Beltrami differential, all of which are subject to certain normalization conditions, spelled out respectively in eq. (3.18), (3.29) and (3.28) of [2]. Note that all quantities in (3.17) are expressed with respect to the period matrix $\Omega_{I J}$.

The last step in the derivation of the consistent and slice-independent measure for the $\mathbf{Z}_{2}$-twisted theory is the change of variables from the bosonic period matrix $\Omega_{I J}$ to the super-period matrix $\hat{\Omega}_{I J}$. The super-period matrix is invariant under local supersymmetry. As was shown in [2], this guarantees the existence of consistent bosonic moduli and permits the consistent integration over odd supermoduli. The reformulation of superstring amplitudes in terms of the super-period matrix is one of the key insights into two-loop superstring perturbation theory presented in $[1,2,3,4]$, and was built on earlier work in $[23,21,37,38]$.

To carry out the change of variables from $\Omega_{I J}$ to $\hat{\Omega}_{I J}$, one proceeds as follows. As explained in [2], §3.3-§3.5, the choice of $\hat{\Omega}_{I J}$ as parameters for the even supermoduli determines the Beltrami superdifferentials $H_{A}$ in the gauge-fixed formulas (1.3) and (3.12). The remaining difficulty is that the string amplitude is still expressed in terms of correlators of conformal field theories with respect to a background metric with period matrix $\Omega_{I J}$ instead of $\hat{\Omega}_{I J}$. Now the difference between the two period matrices is of order two in $\chi$ 
and thus nilpotent. The expansion in terms of a Beltrami differential $\hat{\mu}$ is thus exact to first order,

$$
\hat{\Omega}_{I J}=\Omega_{I J}-i \int d^{2} z \hat{\mu} \omega_{I} \omega_{J}(z)
$$

The process will therefore only affect the terms in (3.17) that are independent of $\chi$. The effect of this change of variables is a change in the worldsheet metric by means of the Beltrami differential $\hat{\mu}$, defined above (up to a diffeomorphism). In any correlator, this change may be implemented via the insertion of the stress tensor, as was explained in [2]. The combination of partition functions and finite-dimensional determinants may be treated by these methods, and we obtain,

$$
\begin{aligned}
& \frac{\left\langle\prod_{a} b\left(p_{a}\right) \prod_{\alpha} \delta\left(\beta\left(q_{\alpha}\right)\right)\right\rangle_{M}}{\operatorname{det} \Phi_{I J+}\left(p_{a}\right) \cdot \operatorname{det}\left\langle H_{\alpha} \mid \Phi_{\beta}^{*}\right\rangle} \frac{Z_{C}[\delta ; \varepsilon]}{Z_{M}[\delta]}(\Omega) \\
& \quad=\frac{\left\langle\prod_{a} b\left(p_{a}\right) \prod_{\alpha} \delta\left(\beta\left(q_{\alpha}\right)\right)\right\rangle_{M}}{\operatorname{det} \Phi_{I J+}\left(p_{a}\right) \cdot \operatorname{det}\left\langle H_{\alpha} \mid \Phi_{\beta}^{*}\right\rangle} \frac{Z_{C}[\delta ; \varepsilon]}{Z_{M}[\delta]}(\hat{\Omega}) \times\left\{1+\int \frac{d^{2} z}{2 \pi} \hat{\mu}(z)\langle T(z)\rangle_{C}\right\}
\end{aligned}
$$

Substituting this result into (3.17) yields

$$
\begin{aligned}
\mathcal{A}_{C}[\delta ; \varepsilon]\left(p_{\varepsilon}\right)= & \frac{\left\langle\prod_{a} b\left(p_{a}\right) \prod_{\alpha} \delta\left(\beta\left(q_{\alpha}\right)\right)\right\rangle_{M}}{\operatorname{det} \Phi_{I J+}\left(p_{a}\right) \cdot \operatorname{det}\left\langle H_{\alpha} \mid \Phi_{\beta}^{*}\right\rangle}(\hat{\Omega}) \frac{Z_{C}[\delta ; \varepsilon]}{Z_{M}[\delta]}(\hat{\Omega}) \exp \left\{i \pi \tilde{\tau}_{\varepsilon} p_{\varepsilon}^{2}\right\} \\
& \times\left\{1-\frac{1}{8 \pi^{2}} \int d^{2} z \int d^{2} w \chi_{\bar{z}}^{+} \chi_{\bar{w}}^{+}\langle S(z) S(w)\rangle_{C}+\int \frac{d^{2} z}{2 \pi} \hat{\mu}(z)\langle T(z)\rangle_{C}\right\}
\end{aligned}
$$

The ghost part of the partition function and of the supercurrent and stress tensor correlators as well as the finite-dimensional determinants in (3.19) are exactly the same as those for the uncompactified theory and given by

$$
\begin{aligned}
\mathcal{A}_{M}[\delta]= & \frac{\left\langle\prod_{a} b\left(p_{a}\right) \prod_{\alpha} \delta\left(\beta\left(q_{\alpha}\right)\right)\right\rangle_{M}}{\operatorname{det} \Phi_{I J+}\left(p_{a}\right) \cdot \operatorname{det}\left\langle H_{\alpha} \mid \Phi_{\beta}^{*}\right\rangle} \\
& \times\left\{1-\frac{1}{8 \pi^{2}} \int d^{2} z \int d^{2} w \chi_{\bar{z}}^{+} \chi_{\bar{w}}^{+}\langle S(z) S(w)\rangle_{M}+\int \frac{d^{2} z}{2 \pi} \hat{\mu}(z)\langle T(z)\rangle_{M}\right\}
\end{aligned}
$$

Here and above, the subscripts $M$ and $C$ are used on the correlators to indicate whether they are evaluated in the flat Minkowski theory $(M)$ or in the compactified orbifold theory $(C)$. The advantage of expressing $\mathcal{A}_{C}[\delta ; \varepsilon]\left(p_{\varepsilon}\right)$ in terms of $\mathcal{A}_{M}[\delta]$ is that the latter has already been explicitly evaluated in [4]. The result is,

$$
\mathcal{A}_{M}[\delta](\Omega)=\mathcal{Z}+\frac{\zeta^{1} \zeta^{2}}{16 \pi^{6}} \cdot \frac{\Xi_{6}[\delta](\Omega) \vartheta[\delta](0, \Omega)^{4}}{\Psi_{10}(\Omega)}
$$


where the normalized partition function $\mathcal{Z}$ of chiral matter, ghosts and superghosts on the bosonic surface with $\chi=0$ is given by

$$
\begin{aligned}
\mathcal{Z} & \equiv \frac{\left\langle\prod_{a} b\left(p_{a}\right) \prod_{\alpha} \delta\left(\beta\left(q_{\alpha}\right)\right)\right\rangle_{M}}{\operatorname{det} \omega_{I} \omega_{J}\left(p_{a}\right)} \\
& =\frac{\vartheta[\delta](0)^{5} \vartheta\left(p_{1}+p_{2}+p_{3}-3 \Delta\right) \prod_{a<b} E\left(p_{a}, p_{b}\right) \prod_{a} \sigma\left(p_{a}\right)^{3}}{Z^{15} \vartheta[\delta]\left(q_{1}+q_{2}-2 \Delta\right) E\left(q_{1}, q_{2}\right) \sigma\left(q_{1}\right)^{2} \sigma\left(q_{2}\right)^{2} \operatorname{det} \omega_{I} \omega_{J}\left(p_{a}\right)}
\end{aligned}
$$

All quantities entering this expression were defined in $\S 2$, except for the chiral scalar partition function $Z^{-1}$ and the holomorphic 1-form $\sigma(z)$, which are defined by

$$
\begin{aligned}
Z^{3} & =\frac{\vartheta\left(z_{1}+z_{2}-w_{0}-\Delta\right) E\left(z_{1}, z_{2}\right) \sigma\left(z_{1}\right) \sigma\left(z_{2}\right)}{\sigma\left(w_{0}\right) E\left(z_{1}, w_{0}\right) E\left(z_{2}, w_{0}\right) \operatorname{det} \omega_{I}\left(z_{J}\right)} \\
\frac{\sigma(z)}{\sigma(w)} & =\frac{\vartheta\left(z-z_{1}-z_{2}+\Delta\right) E\left(w, z_{1}\right) E\left(w, z_{2}\right)}{\vartheta\left(w-z_{1}-z_{2}+\Delta\right) E\left(z, z_{1}\right) E\left(z, z_{2}\right)}
\end{aligned}
$$

In each formula, the points $z_{1}, z_{2}$ and $w_{0}$ are arbitrary.

In terms of $\mathcal{A}_{M}[\delta]$, the following expression is obtained for the chiral measure,

$$
\begin{aligned}
\mathcal{A}_{C}[\delta, \varepsilon]\left(p_{\varepsilon}^{\mu}\right)= & \mathcal{A}_{M}[\delta] \frac{Z_{C}[\delta ; \varepsilon]}{Z_{M}[\delta]} \exp \left\{i \pi \tilde{\tau}_{\varepsilon} p_{\varepsilon}^{2}\right\} \\
& \times\left\{1-\frac{1}{8 \pi^{2}} \int d^{2} z \int d^{2} w \chi_{\bar{z}}^{+} \chi_{\bar{w}}^{+}\left(\left\langle S_{m}(z) S_{m}(w)\right\rangle_{C}-\left\langle S_{m}(z) S_{m}(w)\right\rangle_{M}\right)\right. \\
& \left.\quad+\frac{1}{2 \pi} \int d^{2} z \hat{\mu}(z)\left(\left\langle T_{m}(z)\right\rangle_{C}-\left\langle T_{m}(z)\right\rangle_{M}\right)\right\}
\end{aligned}
$$

Here, we have used the fact that the ghost contributions in the correlators $\langle S(z) S(w)\rangle_{M}$ and $\langle S(z) S(w)\rangle_{C}$ as well as in $\langle T(z)\rangle_{M}$ and $\langle T(z)\rangle_{C}$ are identical and cancel out upon taking differences, leaving only the matter correlators in (3.24), evaluated in the sector twisted by $\varepsilon$. It is understood that all parts of (3.24) are expressed with respect to the super-period matrix $\hat{\Omega}_{I J}$. The only ingredient in (3.24) which needs further clarification is the correction to the Prym period $\tilde{\tau}_{\varepsilon}$, to be presented in the subsequent subsection.

\subsection{The super-Prym period}

In this subsection, the role of the quantity $\tilde{\tau}_{\varepsilon}$ in the exponential involving the internal loop momentum $p_{\varepsilon}$ in (3.24) is clarified. By construction, $\tilde{\tau}_{\varepsilon}$ is invariant under local worldsheet supersymmetry. At first sight, this property would appear to qualify $\tilde{\tau}_{\varepsilon}$ for the supersymmetric generalization of the Prym period $\tau_{\varepsilon}$, but this hypothesis is invalid for the following reasons.

Recall the Schottky relations on a bosonic Riemann surface with period matrix $\Omega_{I J}$, already presented in (1.6) and reformulated in (2.12). The solution of the Schottky relations for $\tau_{\varepsilon}$ as a function of $\Omega$ for a twist $\varepsilon$ was denoted by the function $\tau_{\varepsilon}=R_{\varepsilon}(\Omega)$ 
in (1.6). Actually, this function will be multi-valued because, for given $\Omega$, the Schottky relations determine $\tau$ only up to a shift $\tau_{\varepsilon} \rightarrow \tau_{\varepsilon}+4$. This multivaluedness is required in particular by the fact that the Dehn twist $A_{1} B_{2} A_{1}^{-1} B_{1}^{-1}$, which does not act on $\Omega$, shifts $\tau_{\varepsilon} \rightarrow \tau_{\varepsilon}+4$, as shown in [22].

The implications for the super-Prym period and for the quantity $\tilde{\tau}_{\varepsilon}$ are as follows. The genus 2 super-Riemann surface, specified by the supermoduli $\left(\Omega_{I J}, \zeta^{\alpha}\right)$, uniquely projects to a bosonic Riemann surface with period matrix $\hat{\Omega}_{I J}$. This projection automatically entails an associated super-Prym period $\hat{\tau}_{\varepsilon}$, which is defined through the bosonic Schottky relations from the super-period matrix $\hat{\Omega}_{I J}$. In summary, we have the relations,

$$
\begin{aligned}
\tilde{\tau}_{\varepsilon}-\tau_{\varepsilon} & =-\frac{i}{8 \pi} \int d^{2} z \int d^{2} w \omega_{\varepsilon}(z) \chi_{\bar{z}}^{+} S_{\delta+\epsilon}(z, w) \chi_{\bar{w}}^{+} \omega_{\varepsilon}(w) \\
\hat{\Omega}_{I J}-\Omega_{I J} & =-\frac{i}{8 \pi} \int d^{2} z \int d^{2} w \omega_{I}(z) \chi_{\bar{z}}^{+} S_{\delta}(z, w) \chi_{\bar{w}}^{+} \omega_{J}(w)
\end{aligned}
$$

as well as the defining relations,

$$
\tau_{\varepsilon}=R_{\varepsilon}(\Omega) \quad \hat{\tau}_{\varepsilon}=R_{\varepsilon}(\hat{\Omega})
$$

By their very construction, $\hat{\Omega}, \hat{\tau}$ and $\tilde{\tau}$ are supersymmetric invariant. As a result, the difference

$$
\Delta \tau_{\varepsilon} \equiv \tilde{\tau}_{\varepsilon}-\hat{\tau}_{\varepsilon}=\tilde{\tau}_{\varepsilon}-\tau_{\varepsilon}-\left(\hat{\Omega}_{I J}-\Omega_{I J}\right) \partial_{I J} R_{\varepsilon}(\hat{\Omega})
$$

is also a supersymmetric invariant. This invariance may be verified directly, using the above definitions. Since this expression is bilinear in $\chi$ already, only the supersymmetry variation of $\chi$ is required and this is given by $\delta_{\xi} \chi_{\bar{z}}^{+}=-2 \partial_{\bar{z}} \xi^{+}$, so that

$$
\begin{aligned}
\delta_{\xi} \Delta \tau_{\varepsilon} & =-i \int d^{2} z \xi^{+}(z) \chi_{\bar{z}}^{+}\left[\omega_{\varepsilon}(z) \omega_{\varepsilon}(z)-\omega_{I}(z) \omega_{J}(z) \partial_{I J} R_{\varepsilon}\right] \\
& =-\frac{1}{2 \pi} \int d^{2} z \xi^{+}(z) \chi_{\bar{z}}^{+}\left[\delta_{z z} \tau-\delta_{z z} R_{\varepsilon}\right]
\end{aligned}
$$

which cancels in view of $\tau_{\varepsilon}=R_{\varepsilon}(\Omega)$.

The difference $\Delta \tau_{\varepsilon}$ is non-vanishing. This may be shown by going to split gauge, defined by $\chi_{\bar{z}}^{+}=\sum_{\alpha=1}^{2} \zeta^{\alpha} \delta\left(z, q_{\alpha}\right)$ with $S_{\delta}\left(q_{1}, q_{2}\right)=0$, in which $\hat{\Omega}=\Omega, \hat{\tau}_{\varepsilon}=\tau_{\varepsilon}$, so that

$$
\Delta \tau_{\varepsilon}=-\frac{i \zeta^{1} \zeta^{2}}{4 \pi} \omega_{\varepsilon}\left(q_{1}\right) S_{\delta+\epsilon}\left(q_{1}, q_{2}\right) \omega_{\varepsilon}\left(q_{2}\right)
$$

but this quantity is manifestly non-vanishing when $\varepsilon \neq 0$ and $S_{\delta}\left(q_{1}, q_{2}\right)=0$. 


\section{The Chiral Measure for General Compactifications}

In this section, the chiral superstring measure will be constructed for more general compactifications than those involving $\mathbf{Z}_{2}$ twists. The total space-time for the compactifications considered here will again be denoted by $C$. The chiral measure will be evaluated at fixed even spin structure. As announced in [1], under some basic but mild assumptions, it will be shown that the chiral blocks are independent of any choices of gauge slice, just as they were in flat space-time. The assumptions are

1. The compactification only modifies the matter part of the theory, leaving the superghost part unchanged.

2. The compactification respects local worldsheet supersymmetry, so that the superVirasoro algebra with matter central charge $c=15$ is preserved.

A simple prescription for their calculation will be given first in split gauge and then in terms of the OPE of two supercurrents.

\subsection{The Result of chiral splitting}

Chiral splitting (the fact that the superstring amplitudes are the norms squared of supermeromorphic functions on supermoduli space) holds for the contribution of individual super-conformal families. In the case of the $\mathbf{Z}_{2}$ orbifold measure discussed in $\S 2$, superconformal families were labelled by the spin structure $\delta$, by the twist $\varepsilon$ and by the internal momenta, $p_{I}^{\mu}$ in the untwisted sector and $p_{\varepsilon}^{\mu}$ in the twisted sectors. For more general compactifications, the super-conformal family structure may be more complicated. The super-conformal families will be labeled here by the spin structure $\delta$ and the remaining characterization will be summarized by a label $\lambda$. The spin structure label is singled out here because it must coincide with the spin structure of the ghost part of the measure.

All the serious complications in the derivation of the superstring measure have to do with the gauge fixing, ghost and finite-dimensional determinant contributions. In view of our above assumptions, all these contributions are sensibly the same as in flat Minkowski space-time or in the orbifolded space-times. Thus, the general form of chiral splitting for the superstring measure for strings moving on the compactified space-time $C$ is readily adapted from the expression for the orbifold case in (3.12) at two-loops, ${ }^{9}$

$$
\begin{aligned}
\mathbf{A}_{C}[\delta] & =\sum_{\lambda} \int_{s \mathcal{M}_{2}} \prod_{A}\left|d m^{A}\right|^{2}\left|\mathcal{A}_{C}[\delta ; \lambda]\right|^{2} \\
\mathcal{A}_{C}[\delta ; \lambda] & =\left\langle\prod_{A} \delta\left(\left\langle H_{A} \mid B\right\rangle\right) \prod_{i} V_{i}^{\text {chi }} \exp \left\{\int \frac{d^{2} z}{2 \pi} \chi_{\bar{z}}^{+} S_{C}(z)\right\}\right\rangle_{\lambda,+}
\end{aligned}
$$

\footnotetext{
${ }^{9}$ A subscript $C$ has been appended to the supercurrent $S$ because for general compactifications, the supercurrent may not assume the flat space-time form; the latter will henceforth be denoted by $S_{M}$.
} 
Here, the subscript $\lambda$ refers to the fact that the amplitude is evaluated in the sector associated with the super-conformal family $\lambda$. Notice that, compared to (3.12), no additional factor depending on internal momenta is exhibited. The presence of internal momenta amongst the labels for super-conformal blocks is indeed model dependent and is assumed to be part of the definition of $\langle\cdots\rangle_{\lambda,+}$.

In terms of $\mathcal{A}_{M}[\delta]$, the following expression is obtained for the chiral measure,

$$
\begin{aligned}
\mathcal{A}_{C}[\delta ; \lambda]= & \mathcal{A}_{M}[\delta] \frac{Z_{C}[\delta ; \lambda]}{Z_{M}[\delta]}\left\{1-\frac{1}{2 \pi} \int d^{2} z \hat{\mu}(z)\left\{\left\langle T_{C m}(z)\right\rangle_{C \lambda}-\left\langle T_{M m}(z)\right\rangle_{M}\right\}\right. \\
& \left.-\frac{1}{8 \pi^{2}} \int d^{2} z \int d^{2} w \chi_{\bar{z}}^{+} \chi_{\bar{w}}^{+}\left\{\left\langle S_{C m}(z) S_{C m}(w)\right\rangle_{C \lambda}-\left\langle S_{M m}(z) S_{M m}(w)\right\rangle_{M}\right\}\right\}
\end{aligned}
$$

Here, we have used the fact that the ghost contributions in the correlators $\left\langle S_{M}(z) S_{M}(w)\right\rangle_{M}$ and $\left\langle S_{C}(z) S_{C}(w)\right\rangle_{C \lambda}$ as well as in $\left\langle T_{M}(z)\right\rangle_{M}$ and $\left\langle T_{C}(z)\right\rangle_{C \lambda}$ are identical and cancel out upon taking differences, leaving only the matter correlators in (4.2). It is understood that all parts of (4.2) are expressed with respect to the super-period matrix $\hat{\Omega}_{I J}$.

\subsection{Slice Independence of the Measure for Compactifications}

The slice independence of $\mathcal{A}_{C}[\delta ; \lambda]$ may be deduced from the slice independence of $\mathcal{A}_{M}[\delta]$, which was already established in $[2,3]$, together with general properties of the supercurrent and stress tensor correlators which enter into (4.2). Since the ghost parts have cancelled out of the stress tensor and supercurrent correlators, their singularities with the ghost insertion points have also cancelled. Therefore, both $\left\langle T_{m}(z)\right\rangle_{C \lambda}$ and $\left\langle T_{m}(z)\right\rangle_{M}$ are holomorphic and their difference is a holomorphic 2-form that is well-defined on the Riemann surface, and thus the formula for $\mathcal{A}_{C}[\delta ; \lambda]$ is independent of the choice of $\hat{\mu}$ within a given superconformal family $\lambda$.

The supercurrent insertions are similarly independent of the points $q_{\alpha}$. Since the ghost parts of $S_{C}$ and $S_{M}$ coincide, all the singularities in $z$ and $w$ with the insertion points $p_{a}$ and $q_{\alpha}$ are identical, and cancel upon taking the difference between the $C$ and $M$ contributions. Thus, the only possible singularities in the $S S$ correlator is when $z \rightarrow w$. But this singularity is precisely cancelled by the presence of the stress tensor contribution, as was shown in the flat case in $[2,3]$.

The mutual cancellation of these singularities is also a necessary and sufficient in order to maintain local worldsheet supersymmetry, as shown in [2]. Indeed, each singularity presents an obstruction to supersymmetry invariance since the supersymmetry variation $\delta_{\xi} \chi_{\bar{z}}^{+}=-2 \partial_{\bar{z}} \xi^{+}$will pick up non-vanishing contributions at the poles. Just as in [2] for flat space-time $M$, the effect of the singularity in the supercurrent correlator at $z=w$ is precisely cancelled by the variation $\delta_{\xi} \mu=\xi^{+} \chi_{\bar{z}}^{+}$of the stress tensor term. In summary, the insertion of $\left\langle S_{C m}(z) S_{C m}(w)\right\rangle_{C \lambda}-\left\langle S_{M m}(z) S_{M m}(w)\right\rangle_{M}$ is completely singularity free and hence $\mathcal{A}_{C}[\delta ; \lambda]$ is slice independent, just as $\mathcal{A}_{M}[\delta]$ was. 


\subsection{The Measure for Compactifications in Split Gauge}

To evaluate explicitly the superstring measure, we now choose pointlike insertions for $\chi$

$$
\chi_{\bar{z}}^{+}(z)=\zeta^{1} \delta\left(z, x_{1}\right)+\zeta^{2} \delta\left(z, x_{2}\right)
$$

As in [3], the slice independence of $\mathcal{A}_{C}[\delta ; \lambda]$ guarantees well-defined and regular limits as $x_{\alpha} \rightarrow q_{\alpha}$. We obtain

$$
\begin{gathered}
\mathcal{A}_{C}[\delta ; \lambda]=\mathcal{A}_{M}[\delta] \frac{Z_{C}[\delta ; \lambda]}{Z_{M}[\delta]}\left\{1-\frac{\zeta^{1} \zeta^{2}}{4 \pi^{2}}\left[\left\langle S_{C m}\left(q_{1}\right) S_{C m}\left(q_{2}\right)\right\rangle_{C \lambda}-\left\langle S_{M m}\left(q_{1}\right) S_{M m}\left(q_{2}\right)\right\rangle_{M}\right]\right. \\
\left.+\frac{1}{2 \pi} \sum_{a} \mu_{a}\left(q_{1}, q_{2}\right)\left[\left\langle T_{C m}\left(p_{a}\right)\right\rangle_{C}(f)-\left\langle T_{M m}\left(p_{a}\right)\right\rangle_{M}\right]\right\}
\end{gathered}
$$

where the flat Minkowski space-time objects $\mathcal{A}_{M}[\delta]$ and $\mathcal{Z}$ were given in (3.21) and (3.22). Here, $\mu_{a}$ arises from the Beltrami differential $\hat{\mu}$ representing the shift from the period matrix $\Omega$ to the superperiod matrix $\hat{\Omega}$, given by

$$
\mu_{a}\left(q_{1}, q_{2}\right)=\frac{\zeta^{1} \zeta^{2}}{4 \pi} \varpi_{a}\left(q_{1}, q_{2}\right) S_{\delta}\left(q_{1}, q_{2}\right)
$$

Here, $\varpi_{a}(z, w)$ is the unique form of degree 1 and holomorphic in both $z$ and $w$ such that the 3 holomorphic 2 -forms $\varpi_{a}(z, z)$ are normalized by $\varpi_{a}\left(p_{b}, p_{b}\right)=\delta_{a b}$. Explicit forms were given in [2], eq. (1.15). The difference of the supercurrent and stress tensor correlators on manifolds $C$ and $M$, given by

$$
\begin{aligned}
Q\left(q_{1}, q_{2}\right) \equiv+ & +\left\langle S_{C m}\left(q_{1}\right) S_{C m}\left(q_{2}\right)\right\rangle_{C \lambda}-\left\langle S_{M m}\left(q_{1}\right) S_{M m}\left(q_{2}\right)\right\rangle_{M} \\
& -\frac{1}{2} \sum_{a} \varpi_{a}\left(q_{1}, q_{2}\right) S_{\delta}\left(q_{1}, q_{2}\right)\left[\left\langle T_{C m}\left(p_{a}\right)\right\rangle_{C \lambda}-\left\langle T_{M m}\left(p_{a}\right)\right\rangle_{M}\right]
\end{aligned}
$$

is holomorphic in both $q_{1}$ and $q_{2}$ and odd under the interchange of $q_{1}$ and $q_{2}$. Therefore, its dependence on $q_{\alpha}$ is determined uniquely up to a $q_{\alpha}$-independent multiplicative factor. The expression for $\mathcal{A}_{C}[\delta ; \lambda]$ becomes,

$$
\mathcal{A}_{C}[\delta ; \lambda]=\frac{Z_{C}[\delta ; \lambda]}{Z_{M}[\delta]}\left\{\mathcal{Z}+\frac{\zeta^{1} \zeta^{2}}{16 \pi^{6}} \cdot \frac{\vartheta[\delta](0)^{4} \Xi_{6}[\delta]}{\Psi_{10}}-\frac{\zeta^{1} \zeta^{2}}{4 \pi^{2}} \mathcal{Z} Q\left(q_{1}, q_{2}\right)\right\}
$$

A further simplification takes place in the split gauge, defined by the following relation between the insertion points $q_{1}$ and $q_{2}, S_{\delta}\left(q_{1}, q_{2}\right)=0$. In this gauge, the expression for $\mathcal{A}_{C}[\delta ; \lambda]$ simplifies to

$$
\mathcal{A}_{C}[\delta ; \lambda]=\frac{Z_{C}[\delta ; \lambda]}{Z_{M}[\delta]}\left\{\mathcal{Z}+\frac{\zeta^{1} \zeta^{2}}{16 \pi^{6}} \cdot \frac{\vartheta[\delta](0)^{4} \Xi_{6}[\delta]}{\Psi_{10}}-\frac{\zeta^{1} \zeta^{2}}{4 \pi^{2}} \mathcal{Z}\left\langle S_{C m}\left(q_{1}\right) S_{C m}\left(q_{2}\right)\right\rangle_{C \lambda}\right\} .
$$




\subsection{The Measure via a Leading Supercurrent OPE Operator}

In this subsection, an alternative formula for the chiral measure is provided in terms of simple data that may be obtained from the supercurrent OPE. This calculation may be carried out in terms of the operators $\mathcal{O}_{M}$ and $\mathcal{O}_{C}$, defined as follows,

$$
\begin{aligned}
S_{C}(z) S_{C}(w) & =\frac{1}{4} \frac{T_{C}(z)+T_{C}(w)}{z-w}+(z-w) \mathcal{O}_{C}(w)+\mathcal{O}(z-w)^{2} \\
S_{M}(z) S_{M}(w) & =\frac{1}{4} \frac{T_{M}(z)+T_{M}(w)}{z-w}+(z-w) \mathcal{O}_{M}(w)+\mathcal{O}(z-w)^{2}
\end{aligned}
$$

Notice that the leading cubic singularity cancels since the central charges for $M$ and for $C$ are assumed to be the same, namely $c=15$. A convenient form for the chiral measure based on the above OPE operators is obtained by letting all points $p_{a}$ collapse to the point $q_{2}$ and subsequently letting $q_{1} \rightarrow q_{2}$.

Using methods similar to those employed in [3], section $\S 3.4$, a limiting formula is obtained for the summation against $\mu_{a}$ of the full stress tensor $T\left(p_{a}\right) \equiv\left\langle T_{C m}\left(p_{a}\right)\right\rangle_{C}-$ $\left\langle T_{M m}\left(p_{a}\right)\right\rangle_{M}$ in terms of holomorphic Abelian differentials $\omega_{\alpha}^{*}$ with normalization $\omega_{\alpha}^{*}\left(q_{\beta}\right)=$ $\delta_{\alpha \beta}$. In the OPE relation of (4.9), it is customary to expand with respect to the coordinate $q_{2}$ of the second operator, so we also let $p_{a} \rightarrow q_{2}$ for all $a=1,2,3$. As a result,

$$
\lim _{p_{a} \rightarrow q_{2}} \sum_{a} \varpi_{a}\left(q_{1}, q_{2}\right) T\left(p_{a}\right)=\frac{1}{2} \frac{1}{\partial \omega_{1}^{*}\left(q_{2}\right)} \partial T\left(q_{2}\right)-\frac{\partial \omega_{2}^{*}\left(q_{2}\right)}{\partial \omega_{1}^{*}\left(q_{2}\right)} T\left(q_{2}\right)
$$

Expanding in powers of $q_{2}-q_{1}$ up to and including second order, this limit reduces to

$$
T\left(q_{2}\right)+\frac{1}{2}\left(q_{1}-q_{2}\right) \partial T\left(q_{2}\right)+\left(q_{1}-q_{2}\right)^{2}\left\{\frac{1}{2} f\left(q_{2}\right) \partial T\left(q_{2}\right)-3 T_{0}\left(q_{2}\right) T\left(q_{2}\right)\right\}
$$

Here, we have introduced the following notations, familiar from $[1,2]$

$$
\begin{aligned}
f(w) & =\omega_{I}(w) \partial_{I} \ln \vartheta\left(2 w-w_{0}-\Delta\right)-\partial_{w} \ln E\left(w, w_{0}\right)+\partial_{w} \ln \sigma(w) \\
T_{0}(w) & =\frac{1}{2} \omega_{I}(w) \omega_{J}(w) \partial_{I} \partial_{J} \ln \vartheta\left(2 w-w_{0}-\Delta\right)-\frac{1}{6} \partial f(w)+\frac{1}{6} f(w)^{2}-T_{1}(w) \\
T_{1 / 2}(w) & =\frac{1}{2} \omega_{I}(w) \omega_{J}(w) \partial_{I} \partial_{J} \ln \vartheta[\delta](0)-T_{1}(w)
\end{aligned}
$$

Collecting these results, the expansion up to order $\mathcal{O}\left(q_{1}-q_{2}\right)^{2}$ of $Q$ is given by

$$
\begin{aligned}
Q\left(q_{1}, q_{2}\right)= & +\left\langle S_{C}\left(q_{1}\right) S_{C}\left(q_{2}\right)\right\rangle_{C}-\left\langle S_{M}\left(q_{1}\right) S_{M}\left(q_{2}\right)\right\rangle_{M} \\
& -\frac{1}{2} \frac{\left\langle T\left(q_{2}\right)\right\rangle}{q_{1}-q_{2}}-\frac{1}{4}\left\langle\partial T\left(q_{2}\right)\right\rangle-\frac{1}{2}\left(q_{1}-q_{2}\right)\left[\left(T_{1 / 2}-3 T_{0}\right)\langle T\rangle+\frac{1}{2} f\langle\partial T\rangle\right]\left(q_{2}\right) .
\end{aligned}
$$

It is easy to check that, within this approximation, $Q\left(q_{1}, q_{2}\right)$ is indeed a form of weight $(3 / 2,3 / 2)$, even though individual terms in its expression above do not transform covariantly under conformal reparametrizations $z \rightarrow z^{\prime}=\varphi(z)$. To check this, notice that $S(z)$ 
and $T(z)$ are tensors of weights $3 / 2$ and 2 respectively, while $f, T_{1}, T_{1 / 2}$ and $T_{0}$ transform as connections,

$$
\begin{aligned}
\varphi^{\prime}(z) f^{\prime}\left(z^{\prime}\right) & =f(z)-\frac{3}{2} \frac{\varphi^{\prime \prime}(z)}{\varphi^{\prime}(z)} \\
\varphi^{\prime}(z)^{2} T_{0}^{\prime}\left(z^{\prime}\right) & =T_{0}(z)-\frac{1}{3} \frac{\varphi^{\prime \prime}(z)}{\varphi^{\prime}(z)} f(z)+\frac{1}{6} \frac{\varphi^{\prime \prime \prime}(z)}{\varphi^{\prime}(z)} \\
\varphi^{\prime}(z)^{2} T_{n}^{\prime}\left(z^{\prime}\right) & =T_{n}(z)+\frac{6 n^{2}-6 n+1}{12}\left\{\frac{\varphi^{\prime \prime \prime}(z)}{\varphi^{\prime}(z)}-\frac{3}{2}\left(\frac{\varphi^{\prime \prime}(z)}{\varphi^{\prime}(z)}\right)^{2}\right\} \quad n=1 / 2,1
\end{aligned}
$$

Assuming that the OPE of two supercurrents is as given in (4.9), we get

$$
\begin{aligned}
Q\left(q_{1}, q_{2}\right) & =\left(q_{1}-q_{2}\right) \hat{Q}\left(q_{2}\right)+\mathcal{O}\left(q_{1}-q_{2}\right)^{2} \\
\hat{Q}(w) & =\left[\left\langle\mathcal{O}_{C}\right\rangle_{C}-\left\langle\mathcal{O}_{M}\right\rangle_{M}-\frac{1}{8}\left\langle\partial^{2} T\right\rangle-\frac{1}{2}\left(T_{1 / 2}-3 T_{0}\right)\langle T\rangle-\frac{1}{4} f\langle\partial T\rangle\right](w)
\end{aligned}
$$

It remains to evaluate $\mathcal{Z} Q\left(q_{1}, q_{2}\right)$. Since this quantity is independent of both $q$ 's, we first let $q_{1} \rightarrow q_{2}$ and then set $q_{2}=p_{3}=\Delta+\nu_{3}$. The quantity may now be evaluated using the methods developed for flat space-time, and we find

$$
\mathcal{Z} Q\left(q_{1}, q_{2}\right)=\frac{\vartheta[\delta](0, \Omega)^{8}}{\mathcal{M}_{\nu_{1} \nu_{2}}^{2}} \cdot \frac{\hat{Q}\left(p_{3}\right)}{\omega_{\nu_{1}}\left(p_{3}\right)^{2} \omega_{\nu_{2}}\left(p_{3}\right)^{2}}
$$

Substituting this result into the measure factor, we obtain the chiral measure

$$
\mathcal{A}_{C}=\frac{Z_{C}}{Z_{M}}\left\{\mathcal{Z}+\frac{\zeta^{1} \zeta^{2}}{16 \pi^{6}} \cdot \frac{\vartheta[\delta]^{4} \Xi_{6}[\delta]}{\Psi_{10}}-\frac{\zeta^{1} \zeta^{2}}{4 \pi^{2}} \cdot \frac{\vartheta[\delta]^{8}}{\mathcal{M}_{\nu_{1} \nu_{2}}^{2}} \cdot \frac{\hat{Q}\left(p_{3}\right)}{\omega_{\nu_{1}}\left(p_{3}\right)^{2} \omega_{\nu_{2}}\left(p_{3}\right)^{2}}\right\},
$$

as well as the contribution to the cosmological constant from the left-moving sector, by integrating over $\zeta^{\alpha}$.

\section{Calculation of two-loop chiral blocks for $\mathrm{Z}_{2}$ twists}

The starting point is the chiral measure of (3.24), evaluated in split gauge defined by $S_{\delta}\left(q_{1}, q_{2}\right)=0$. In this gauge, the following simplifications occur : $\hat{\Omega}_{I J}=\Omega_{I J}$, and thus $\hat{\mu}=$ 0 and the matter supercurrent correlator in $M$ vanishes because the fermion propagator $S_{\delta}$ is evaluated between the points $q_{1}$ and $q_{2}$. The remaining expression is given by, ${ }^{10}$

$$
\mathcal{A}_{C}[\delta ; \varepsilon]\left(p_{\varepsilon}\right)=\frac{Z_{C}[\delta ; \varepsilon]}{Z_{M}[\delta]} e^{i \pi\left(\tau_{\varepsilon}+\Delta \tau_{\varepsilon}\right) p_{\varepsilon}^{2}}\left\{\mathcal{Z}+\frac{\zeta^{1} \zeta^{2}}{16 \pi^{6}} \frac{\Xi_{6}[\delta] \vartheta[\delta](0)^{4}}{\Psi_{10}}-\frac{\zeta^{1} \zeta^{2}}{4 \pi^{2}} \mathcal{Z}\left\langle S_{m}\left(q_{1}\right) S_{m}\left(q_{2}\right)\right\rangle_{\varepsilon}\right\}
$$

\footnotetext{
${ }^{10}$ For $\mathbf{Z}_{2}$-twisting, the supercurrents $S_{C}$ and $S_{M}$ take on the same functional form (as do the stress tensors $T_{C}$ and $T_{M}$ ); thus, the subscripts $M$ and $C$ will be dropped from the operators. The subscript $C$ on the correlator will be replaced with the twist $\varepsilon$ for each twisted sector.
} 
where $\mathcal{Z}$ was defined in (3.22). As this formula is written in split gauge, we have $\hat{\Omega}_{I J}=\Omega_{I J}$, $\hat{\tau}_{\varepsilon}=\tau_{\varepsilon}$ and the expression for $\Delta \tau_{\varepsilon}$, derived in (3.29), is

$$
\Delta \tau_{\varepsilon}=-\frac{i \zeta^{1} \zeta^{2}}{4 \pi} \omega_{\varepsilon}\left(q_{1}\right) S_{\delta+\epsilon}\left(q_{1}, q_{2}\right) \omega_{\varepsilon}\left(q_{2}\right)
$$

The focus of this paper will be on the chiral measure and the cosmological constant, both of which receive contributions only from the top term in $\zeta^{1} \zeta^{2}$. The resulting chiral measure takes the following form,

$$
\begin{aligned}
d \mu_{C}[\delta ; \varepsilon]\left(p_{\varepsilon}\right) & \equiv \int d \zeta^{2} d \zeta^{1} \mathcal{A}_{C}[\delta ; \varepsilon]\left(p_{\varepsilon}\right) \\
& =e^{i \pi \tau_{\varepsilon} p_{\varepsilon}^{2}} \frac{Z_{C}[\delta, \varepsilon]}{Z_{M}[\delta]}\left\{\frac{\Xi_{6}[\delta] \vartheta[\delta](0)^{4}}{16 \pi^{6} \Psi_{10}}-\frac{\mathcal{Z}}{4 \pi^{2}}\left\langle S_{m}\left(q_{1}\right) S_{m}\left(q_{2}\right)\right\rangle_{\varepsilon}+i \pi p_{\varepsilon}^{2} \Gamma[\delta, \varepsilon]\right\}
\end{aligned}
$$

where the following definition has been made,

$$
\Gamma[\delta ; \varepsilon] \equiv \mathcal{Z} \int d \zeta^{2} d \zeta^{1} \Delta \tau_{\varepsilon}=-\frac{i \mathcal{Z}}{4 \pi} \omega_{\varepsilon}\left(q_{1}\right) S_{\delta+\epsilon}\left(q_{1}, q_{2}\right) \omega_{\varepsilon}\left(q_{2}\right)
$$

It remains to calculate the various terms in the above expression in terms of $\vartheta$-functions, which will be the subject of the remainder of this section.

It will be assumed that $n$ dimensions are being $\mathbf{Z}_{2}$-twisted, leaving $10-n$ dimensions untwisted, and that $n$ is at most 8 . In this case, only even spin structures $\delta$ need to be taken into account. In particular, this will be the case for the models of [9]. The chiral partition functions $Z_{M}$ and $Z_{C}$ are well-known, and given by

$$
\begin{aligned}
Z_{M}[\delta] & =\left(\frac{\vartheta[\delta](0, \Omega)}{Z^{3}}\right)^{5} \\
Z_{C}[\delta ; \varepsilon] & =\left(\frac{\vartheta[\delta](0, \Omega)}{Z^{3}}\right)^{5-n / 2}\left(\frac{\vartheta\left[\delta_{j}^{+}\right](0, \Omega) \vartheta\left[\delta_{j}^{-}\right](0, \Omega) \vartheta[\delta+\varepsilon](0, \Omega)}{Z^{3} \vartheta_{j}\left(0, \tau_{\varepsilon}\right)^{2}}\right)^{n / 2}
\end{aligned}
$$

for any pair $\delta_{j}^{ \pm}$such that $\varepsilon=-\delta_{j}^{+}+\delta_{j}^{-}$.

\subsection{Calculation of the supercurrent correlator}

The supercurrent correlator may be calculated in terms of the twisted scalar and fermion propagators evaluated in section $\S 2$,

$$
\left\langle S_{m}\left(q_{1}\right) S_{m}\left(q_{2}\right)\right\rangle_{\varepsilon}=\frac{n}{4} B_{\varepsilon}\left(q_{1}, q_{2}\right) S_{\delta+\varepsilon}\left(q_{1}, q_{2}\right)
$$

where the twisted scalar propagator is

$$
B_{\varepsilon}(z, w)=S_{\delta_{j}^{+}}(z, w) S_{\delta_{j}^{-}}(z, w)+b_{j} \omega_{\varepsilon}(z) \omega_{\varepsilon}(w)
$$

for any pair $\delta_{j}^{ \pm}$such that $\varepsilon=-\delta_{j}^{+}+\delta_{j}^{-}$. 
For given $\varepsilon \neq 0$, non-vanishing contributions will arise only from $[\delta ; \varepsilon]$ where both $\delta$ and $\delta+\varepsilon$ are even characteristics. Upon choosing $\delta=\delta_{i}^{+}$(the choice $\delta=\delta_{i}^{-}$leads to the same result), we have

$$
B_{\varepsilon}\left(q_{1}, q_{2}\right)=S_{\delta}\left(q_{1}, q_{2}\right) S_{\delta+\varepsilon}\left(q_{1}, q_{2}\right)+b_{i} \omega_{\varepsilon}\left(q_{1}\right) \omega_{\varepsilon}\left(q_{2}\right)
$$

As $q_{1}, q_{2}$ obey the split gauge relation $S_{\delta}\left(q_{1}, q_{2}\right)=0$, the first term on the rhs above vanishes and we have $B_{\varepsilon}\left(q_{1}, q_{2}\right)=b_{i} \omega_{\varepsilon}\left(q_{1}\right) \omega_{\varepsilon}\left(q_{2}\right)$. Using the explicit expression for $b_{i}$, computed in (2.27), we have

$$
\left\langle S_{m}\left(q_{1}\right) S_{m}\left(q_{2}\right)\right\rangle_{\varepsilon}=-i \pi n \omega_{\varepsilon}\left(q_{1}\right) \omega_{\varepsilon}\left(q_{2}\right) S_{\delta_{i}^{-}}\left(q_{1}, q_{2}\right) \partial_{\tau_{\varepsilon}} \ln \vartheta_{i}\left(0, \tau_{\varepsilon}\right)
$$

The combination of this correlator with the factor of $\mathcal{Z}$ may be re-expressed conveniently in terms of $\Gamma[\delta ; \varepsilon]$,

$$
\mathcal{Z}\left\langle S_{m}\left(q_{1}\right) S_{m}\left(q_{2}\right)\right\rangle_{\varepsilon}=4 \pi^{2} n \Gamma[\delta ; \varepsilon] \partial_{\tau_{\varepsilon}} \ln \vartheta_{i}\left(0, \tau_{\varepsilon}\right)
$$

This leads to the following formula for the chiral measure in terms of $\Gamma[\delta ; \varepsilon]$,

$$
d \mu_{C}[\delta ; \varepsilon]\left(p_{\varepsilon}\right)=e^{i \pi \tau_{\varepsilon} p_{\varepsilon}^{2}} \frac{Z_{C}[\delta, \varepsilon]}{Z_{M}[\delta]}\left\{\frac{\Xi_{6}[\delta] \vartheta[\delta](0)^{4}}{16 \pi^{6} \Psi_{10}}+\left(i \pi p_{\varepsilon}^{2}-n \partial_{\tau_{\varepsilon}} \ln \vartheta_{i}\left(0, \tau_{\varepsilon}\right)\right) \Gamma[\delta ; \varepsilon]\right\}
$$

It only remains to evaluate $\Gamma[\delta ; \varepsilon]$.

\subsection{Calculation of $\Gamma[\delta ; \varepsilon]$}

In the present subsection, $\Gamma[\delta ; \varepsilon]$ will be evaluated in terms of $\vartheta$-constants. The calculation will be carried out in split gauge, just as for the chiral measure in flat space-time [4]. The key challenge presented by the calculation of $\Gamma[\delta ; \varepsilon]$ is its overall sign. This sign is uniquely fixed by the definition of $\Gamma[\delta ; \varepsilon]$, but during the course of the evaluation, a number of nonintrinsic signs appear and need to be determined. For example, the $\vartheta$-constant itself $\vartheta[\kappa](0)$ may change sign when a full period is added to $\kappa$.

Careful choices for the twist and spin structure assignments are needed, not just for their expression congruent mod 1, but including full periods if they arise as well. The choices made for this calculation are those given in (2.35) where

$$
\begin{aligned}
\varepsilon=-\nu_{a}+\nu_{b} \quad & \delta_{i}^{+}=\nu_{a}+\nu_{c}+\nu_{d} \\
\delta+\varepsilon & =\delta_{i}^{-}=\nu_{b}+\nu_{c}+\nu_{d}
\end{aligned}
$$

By choosing (abcdef) to be an appropriate permutation of (123456), any twist and spin structure assignment may be reached by these conventions. 
The general expression for $\Gamma[\delta ; \varepsilon]$ was given in (5.4); it involves the partition function $\mathcal{Z}$ which was given in (3.22). Using the expression for $\mathcal{Z}$ in split gauge, calculated in eq (3.15) of [4], we obtain

$$
\begin{aligned}
\Gamma[\delta, \varepsilon] & \equiv-\frac{i}{4 \pi} \mathcal{Z} \omega_{\varepsilon}\left(q_{1}\right) S_{\delta_{i}^{-}}\left(q_{1}, q_{2}\right) \omega_{\varepsilon}\left(q_{2}\right) \\
\mathcal{Z} & =-\frac{C}{C_{r}^{2} C_{s}^{2}} \cdot \frac{\vartheta[\delta]^{5} E\left(p_{r}, p_{s}\right)^{4} \sigma\left(p_{r}\right)^{2} \sigma\left(p_{s}\right)^{2}}{\vartheta[\delta]\left(q_{1}+q_{2}-2 \Delta\right) E\left(q_{1}, q_{2}\right) \sigma\left(q_{1}\right)^{2} \sigma\left(q_{2}\right)^{2}} \cdot \frac{1}{\mathcal{M}_{\nu_{r} \nu_{s}}^{2}}
\end{aligned}
$$

where $p_{r}=\nu_{r}+\Delta$ and $p_{s}=\nu_{s}+\Delta$ are two arbitrary branch points. The exponential factors were also introduced in [4] and are given by

$$
\begin{aligned}
C & =-\exp \left\{-4 \pi i \nu_{l}^{\prime}\left(2 \Omega \nu_{r}^{\prime}+2 \nu_{r}^{\prime \prime}\right)\right\}=-\exp \left\{-8 \pi i \nu_{s}^{\prime} \Omega \nu_{r}^{\prime}\right\} \\
C_{r, s} & =\exp \left\{-\pi i \nu_{r, s}^{\prime} \Omega \nu_{r, s}^{\prime}-2 \pi i \nu_{r, s}^{\prime} \nu_{r, s}^{\prime \prime}\right\}
\end{aligned}
$$

We shall make use of the following expression, derived in (2.49), for the product of the Prym differentials at two different points $z, w$,

$$
\omega_{\varepsilon}(z) \omega_{\varepsilon}(w)=-\sigma\left(\mu_{i}, \mu_{j}\right) \frac{1}{\pi^{2} \vartheta_{k}^{4}}\left(S_{\delta_{i}^{+}}(z, w) S_{\delta_{i}^{-}}(z, w)-S_{\delta_{j}^{+}}(z, w) S_{\delta_{j}^{-}}(z, w)\right)
$$

In view of the earlier choice $\delta=\delta_{i}^{+}$, and the split gauge condition $S_{\delta}\left(q_{1}, q_{2}\right)=0$, the above formula simplifies when $z=q_{1}$ and $w=q_{2}$,

$$
\omega_{\varepsilon}\left(q_{1}\right) \omega_{\varepsilon}\left(q_{2}\right)=\sigma\left(\mu_{i}, \mu_{j}\right) \frac{1}{\pi^{2} \vartheta_{k}^{4}} S_{\delta_{j}^{+}}\left(q_{1}, q_{2}\right) S_{\delta_{j}^{-}}\left(q_{1}, q_{2}\right)
$$

Expressions in terms of $\vartheta$-constants are most easily obtained by placing insertion points at branch points. If $q_{1}, q_{2}$ are in split gauge, then their limits to branch points are such that $q_{1}$ and $q_{2}$ belong to the same set in the partition of all six branch points associated with $\delta$. The key difficulty in the evaluation of $\Gamma[\delta ; \varepsilon]$ is that $\mathcal{Z}$ diverges as the points $q_{1}$ and $q_{2}$, are taken to branch points, since $\vartheta[\delta]\left(q_{1}+q_{2}-2 \Delta\right)$ vanish. It turns out that one of the three Szego kernels arising in $\Gamma[\delta ; \varepsilon]$ also vanishes, thereby making their ratio finite.

\subsubsection{Linearization of split gauge around branch points}

To circumvent the above problem, we parametrize the points $q_{1}$ and $q_{2}$ as follows (here the argument is similar to [4], §3.6.2),

$$
\begin{aligned}
& q_{1}(t)=p_{c}+t \dot{q}_{1}+\mathcal{O}\left(t^{2}\right) \\
& q_{2}(t)=p_{d}+t \dot{q}_{2}+\mathcal{O}\left(t^{2}\right)
\end{aligned}
$$


The split gauge relation between $q_{1}$ and $q_{2}$ is clearly obeyed at the point $t=0$, since $\vartheta[\delta]\left(\nu_{c}-\nu_{d}\right) \sim \vartheta\left[\nu_{a}\right]=0$. The vanishing factors in the numerator and denominator are

$$
\begin{aligned}
S_{\delta+\epsilon}\left(p_{c}, p_{d}\right) \sim \vartheta[\delta+\varepsilon]\left(\nu_{c}-\nu_{d}\right) & \sim \vartheta\left[\nu_{b}\right]=0 \\
\vartheta[\delta]\left(p_{c}+p_{d}-2 \Delta\right)=\vartheta[\delta]\left(\nu_{c}+\nu_{d}\right) \sim \vartheta\left[\nu_{a}\right] & =0
\end{aligned}
$$

The split gauge condition to linear order in $t$ yields a non-trivial condition on the growths $\dot{q}_{1}$ and $\dot{q}_{2}$, given by

$$
\dot{q}_{1} \omega_{\nu_{a}}\left(p_{c}\right)-\dot{q}_{2} \omega_{\nu_{a}}\left(p_{d}\right)=0
$$

Here and below, the following notation is used for holomorphic Abelian differentials with double zeros (at the branch points),

$$
\omega_{\nu}(z) \equiv \omega_{I}(z) \partial_{I} \vartheta[\nu](0)
$$

This notation was introduced in [4], eq (2.40). It was also shown there that ratios of these differentials for different $\nu$ 's evaluated at the same branch point may be expressed in terms of $\vartheta$-constants, via the relation

$$
\frac{\omega_{\nu_{i}}\left(p_{k}\right)}{\omega_{\nu_{j}}\left(p_{k}\right)}=\frac{\mathcal{M}_{\nu_{i} \nu_{k}}}{\mathcal{M}_{\nu_{j} \nu_{k}}}
$$

where $\mathcal{M}_{\nu \nu^{\prime}}$ was introduced in [4] and reproduced in (2.44). It was also shown in [4] that $\mathcal{M}_{\nu \nu^{\prime}}$ has the following expression in terms of $\vartheta$-constants for even spin structures,

$$
\mathcal{M}_{\nu_{1}, \nu_{2}}^{2}=\pi^{4} \prod_{k=3,4,5,6} \vartheta\left[\nu_{1}+\nu_{2}+\nu_{k}\right](0)^{2}
$$

where $\nu_{i}, i=1, \cdots, 6$ are all six distinct odd spin structures.

\subsubsection{Evaluation at the branch points}

The ratio of the two vanishing factors may be computed in the limit of vanishing $t$, with the following result,

$$
\begin{aligned}
\frac{\vartheta[\delta+\varepsilon]\left(q_{1}-q_{2}\right)}{\vartheta[\delta]\left(q_{1}+q_{2}-2 \Delta\right)} & =\frac{\left\{\dot{q}_{1} \omega_{I}\left(p_{c}\right)-\dot{q}_{2} \omega_{I}\left(p_{d}\right)\right\} \partial_{I} \vartheta[\delta+\varepsilon]\left(\nu_{c}-\nu_{d}\right)}{\left\{\dot{q}_{1} \omega_{I}\left(p_{c}\right)+\dot{q}_{2} \omega_{I}\left(p_{d}\right)\right\} \partial_{I} \vartheta[\delta]\left(\nu_{c}+\nu_{d}\right)} \\
& =\frac{\left\{\omega_{\nu_{a}}\left(p_{d}\right) \omega_{I}\left(p_{c}\right)-\omega_{\nu_{a}}\left(p_{c}\right) \omega_{I}\left(p_{d}\right)\right\} \partial_{I} \vartheta[\delta+\varepsilon]\left(\nu_{c}-\nu_{d}\right)}{\left\{\omega_{\nu_{a}}\left(p_{d}\right) \omega_{I}\left(p_{c}\right)+\omega_{\nu_{a}}\left(p_{c}\right) \omega_{I}\left(p_{d}\right)\right\} \partial_{I} \vartheta[\delta]\left(\nu_{c}+\nu_{d}\right)}
\end{aligned}
$$

The passage from the first to the second line in the above formula is made using the relation between $\dot{q}_{1}$ and $\dot{q}_{2}$ of (5.20). Next, we have the following relations,

$$
\begin{aligned}
\omega_{I}(z) \partial_{I} \vartheta[\delta+\varepsilon]\left(\nu_{c}-\nu_{d}\right) & =K_{1} \omega_{\nu_{b}}(z) \\
\omega_{I}(z) \partial_{I} \vartheta[\delta]\left(\nu_{c}+\nu_{d}\right) & =K_{2} \omega_{\nu_{a}}(z)
\end{aligned}
$$


where the exponential factors $K_{1}$ and $K_{2}$ will be computed later. In terms of these quantities, we have

$$
\frac{\vartheta[\delta+\varepsilon]\left(q_{1}-q_{2}\right)}{\vartheta[\delta]\left(q_{1}+q_{2}-2 \Delta\right)}=\frac{K_{1}}{2 K_{2}}\left(\frac{\omega_{\nu_{b}}\left(p_{c}\right)}{\omega_{\nu_{a}}\left(p_{c}\right)}-\frac{\omega_{\nu_{b}}\left(p_{d}\right)}{\omega_{\nu_{a}}\left(p_{d}\right)}\right)=-\frac{K_{1}}{2 K_{2}} \frac{\mathcal{M}_{a b} \mathcal{M}_{c d}}{\mathcal{M}_{a c} \mathcal{M}_{a d}}
$$

To pass to the last line, (5.21) has been used to express the ratio of $\omega$ 's in terms of $\mathcal{M}_{\nu \nu^{\prime}}$ 's, as well as the following algebraic relation, $\mathcal{M}_{b c} \mathcal{M}_{a d}-\mathcal{M}_{a c} \mathcal{M}_{b d}=-\mathcal{M}_{a b} \mathcal{M}_{c d}$, which readily follows from the definition of $\mathcal{M}_{\nu \nu^{\prime}}$, given in (2.44).

\subsubsection{Expression in terms of $\vartheta$-constants}

Assembling the expression in (5.13) and (5.16), $\Gamma[\delta ; \varepsilon]$ takes the following form

$$
\begin{aligned}
& \Gamma[\delta ; \varepsilon]=\sigma\left(\mu_{i},\right.\left.\mu_{j}\right) \frac{i}{4 \pi^{3} \vartheta_{k}^{4}} \cdot \frac{C}{C_{r}^{2} C_{s}^{2}} \cdot \frac{\vartheta[\delta]^{5} E\left(p_{r}, p_{s}\right)^{4} \sigma\left(p_{r}\right)^{2} \sigma\left(p_{s}\right)^{2}}{\vartheta[\delta]\left(q_{1}+q_{2}-2 \Delta\right) E\left(q_{1}, q_{2}\right) \sigma\left(q_{1}\right)^{2} \sigma\left(q_{2}\right)^{2}} \\
& \times \frac{1}{\mathcal{M}_{\nu_{r} \nu_{s}}^{2}} S_{\delta_{j}^{+}}\left(q_{1}, q_{2}\right) S_{\delta_{j}^{-}}\left(q_{1}, q_{2}\right) S_{\delta_{i}^{-}}\left(q_{1}, q_{2}\right)
\end{aligned}
$$

Using (5.25), the limit $q_{1} \rightarrow p_{c}=p_{r}, q_{2} \rightarrow p_{d}=p_{s}$ may be safely taken. Next, the expression for the Szegö kernel in terms of $\vartheta$-functions and the prime form is used. All factors of $\sigma$ and $E\left(p_{c}, p_{d}\right)$ now cancel and one obtains,

$$
\Gamma[\delta ; \varepsilon]=\frac{i}{8 \pi^{3}} \frac{\sigma\left(\mu_{i}, \mu_{j}\right)}{\vartheta_{k}^{4}} \frac{C K_{1}}{C_{c}^{2} C_{d}^{2} K_{2}} \frac{\mathcal{M}_{a b}}{\mathcal{M}_{a c} \mathcal{M}_{c d} \mathcal{M}_{d a}} \frac{\vartheta[\delta](0)^{5} \vartheta\left[\delta_{j}^{+}\right]\left(\nu_{c}-\nu_{d}\right) \vartheta\left[\delta_{j}^{-}\right]\left(\nu_{c}-\nu_{d}\right)}{\vartheta\left[\delta_{j}^{+}\right](0) \vartheta\left[\delta_{j}^{-}\right](0) \vartheta\left[\delta_{i}^{-}\right](0)}
$$

It remains to cast this expression in terms of standard $\vartheta$-constants. To this end, we recast some of the factors using the following relations between $\vartheta$-constants.

$$
\begin{aligned}
& \vartheta\left[\delta_{j}^{+}\right]\left(\nu_{c}-\nu_{d}\right)=K_{3} \vartheta\left[\delta_{k}^{+}\right](0) \\
& \vartheta\left[\delta_{j}^{-}\right]\left(\nu_{c}-\nu_{d}\right)=K_{4} \vartheta\left[\delta_{k}^{-}\right](0)
\end{aligned}
$$

The exponential factors $K_{3}$ and $K_{4}$ will be evaluated later. The final result for $\Gamma[\delta ; \varepsilon]$ is now obtained as follows,

$$
\Gamma[\delta, \varepsilon]=\frac{i}{8 \pi^{7}} \frac{\sigma\left(\mu_{i}, \mu_{j}\right)}{\vartheta_{k}^{4}} \cdot \kappa \cdot \kappa^{\prime} \cdot \frac{\vartheta[\delta]^{2}}{\vartheta\left[\delta_{j}^{+}\right]^{2} \vartheta\left[\delta_{j}^{-}\right]^{2} \vartheta[\delta+\varepsilon]^{2}}
$$

where the following definitions have been made,

$$
\begin{aligned}
\kappa & \equiv \frac{C K_{1} K_{3} K_{4}}{C_{c}^{2} C_{d}^{2} K_{2}} \\
\kappa^{\prime} & \equiv \frac{\pi^{4} \mathcal{M}_{a b}}{\mathcal{M}_{a c} \mathcal{M}_{c d} \mathcal{M}_{d a}} \cdot \vartheta[\delta]^{3} \vartheta\left[\delta_{k}^{+}\right] \vartheta\left[\delta_{k}^{-}\right] \vartheta\left[\delta_{j}^{+}\right] \vartheta\left[\delta_{j}^{-}\right] \vartheta\left[\delta_{i}^{-}\right]
\end{aligned}
$$

The combinations $\kappa$ and $\kappa^{\prime}$ will be evaluated in the subsequent subsection, resulting in $\kappa= \pm 1$ and $\kappa^{\prime}= \pm 1$. 


\subsection{Calculation of the overall sign of $\Gamma[\delta ; \varepsilon]$}

Neither $\kappa$, nor $\kappa^{\prime}$ is intrinsic, but the product $\sigma\left(\mu_{i}, \mu_{j}\right) \kappa \kappa^{\prime}$ will be, as will be evidenced by the fact that the final expression for $\Gamma[\delta ; \varepsilon]$ is in terms of squares of $\vartheta$-functions only.

\subsubsection{Calculating $\kappa$}

Let us summarize the definitions of the factors entering into $\kappa$,

$$
\begin{aligned}
C & =-\exp \left\{-8 \pi i \nu_{c}^{\prime} \Omega \nu_{d}^{\prime}\right\} \\
C_{c} & =\exp \left\{-\pi i \nu_{c}^{\prime} \Omega \nu_{c}^{\prime}-2 \pi i \nu_{c}^{\prime} \nu_{c}^{\prime \prime}\right\} \\
C_{d} & =\exp \left\{-\pi i \nu_{d}^{\prime} \Omega \nu_{d}^{\prime}-2 \pi i \nu_{d}^{\prime} \nu_{d}^{\prime \prime}\right\} \\
K_{1} \omega_{\nu_{b}}(z) & =\omega_{I}(z) \partial_{I} \vartheta[\delta+\varepsilon]\left(\nu_{c}-\nu_{d}\right) \\
K_{2} \omega_{\nu_{a}}(z) & =\omega_{I}(z) \partial_{I} \vartheta[\delta]\left(\nu_{c}+\nu_{d}\right) \\
K_{3} \vartheta\left[\delta_{k}^{+}\right](0) & =\vartheta\left[\delta_{j}^{+}\right]\left(\nu_{c}-\nu_{d}\right) \\
K_{4} \vartheta\left[\delta_{k}^{-}\right](0) & =\vartheta\left[\delta_{j}^{-}\right]\left(\nu_{c}-\nu_{d}\right)
\end{aligned}
$$

The $K$-factors may be computed starting from the following basic formula of (B.9),

$$
\vartheta[\delta]\left(z+\Omega \rho^{\prime}+\rho^{\prime \prime}\right)=\vartheta[\delta+\rho](z) \exp \left\{-i \pi \rho^{\prime} \Omega \rho^{\prime}-2 \pi i \rho^{\prime}\left(z+\delta^{\prime \prime}+\rho^{\prime \prime}\right)\right\}
$$

One finds,

$$
\begin{aligned}
& K_{1}=-\exp \left\{-i \pi\left(\nu_{c}-\nu_{d}\right)^{\prime} \Omega\left(\nu_{c}-\nu_{d}\right)^{\prime}-2 \pi i\left(\nu_{c}-\nu_{d}\right)^{\prime} \nu_{b}^{\prime \prime}+4 \pi i \nu_{d}^{\prime} \nu_{c}^{\prime \prime}+4 \pi i \nu_{b}^{\prime} \nu_{c}^{\prime \prime}\right\} \\
& K_{2}=-\exp \left\{-i \pi\left(\nu_{c}+\nu_{d}\right)^{\prime} \Omega\left(\nu_{c}+\nu_{d}\right)^{\prime}+2 \pi i\left(\nu_{c}+\nu_{d}\right)^{\prime} \nu_{a}^{\prime \prime}\right\} \\
& K_{3}=\exp \left\{-i \pi\left(\nu_{c}-\nu_{d}\right)^{\prime} \Omega\left(\nu_{c}-\nu_{d}\right)^{\prime}+2 \pi i\left(\nu_{c}-\nu_{d}\right)^{\prime}\left(\nu_{b}+\nu_{d}+\nu_{f}\right)^{\prime \prime}\right\} \\
& K_{4}=\exp \left\{-i \pi\left(\nu_{c}-\nu_{d}\right)^{\prime} \Omega\left(\nu_{c}-\nu_{d}\right)^{\prime}+2 \pi i\left(\nu_{c}-\nu_{d}\right)^{\prime}\left(\nu_{a}+\nu_{d}+\nu_{f}\right)^{\prime \prime}\right\}
\end{aligned}
$$

Notice that under $c \leftrightarrow d, K_{1} \rightarrow-K_{1}$ while $K_{2} \rightarrow+K_{2}$, even though these symmetries are not manifest. Multiplying all factors yields

$$
\kappa=\frac{C K_{1} K_{3} K_{4}}{C_{c}^{2} C_{d}^{2} K_{2}}=\exp 4 \pi i\left(\nu_{d}^{\prime} \nu_{c}^{\prime \prime}+\nu_{c}^{\prime} \nu_{d}^{\prime \prime}-\nu_{d}^{\prime} \nu_{a}^{\prime \prime}+\nu_{c}^{\prime} \nu_{f}^{\prime \prime}-\nu_{d}^{\prime} \nu_{f}^{\prime \prime}+\nu_{b}^{\prime} \nu_{c}^{\prime \prime}\right)
$$

which indeed takes the values \pm 1 .

\subsubsection{Calculating $\kappa^{\prime}$}

A direct calculation of $\kappa^{\prime}$ from its definition

$$
\kappa^{\prime} \equiv \frac{\pi^{4} \mathcal{M}_{a b}}{\mathcal{M}_{a c} \mathcal{M}_{c d} \mathcal{M}_{d a}} \vartheta[\delta]^{2} \vartheta\left[\delta_{i}^{+}\right] \vartheta\left[\delta_{i}^{-}\right] \vartheta\left[\delta_{j}^{+}\right] \vartheta\left[\delta_{j}^{-}\right] \vartheta\left[\delta_{k}^{+}\right] \vartheta\left[\delta_{k}^{-}\right]
$$

involves the non-intrinsic sign factors, and must be computed case by case. To simplify the process, we shall carry out this calculation for a single non-trivial twist and obtain the 
expression for $\Gamma[\delta ; \varepsilon]$ for all twists by modular invariance. This choice uniquely determines $\nu_{a}$ and $\nu_{b}$ (up to interchange of $a$ and $b$ ). The choice made here is the standard one,

$$
\varepsilon=\left(\begin{array}{l|l}
0 & 0 \\
0 & \frac{1}{2}
\end{array}\right) \quad \nu_{a}=\nu_{2}, \quad \nu_{b}=\nu_{4}
$$

The even spin structures are fixed through the choice of the odd spin structures and (2.35).

The calculation of $\kappa^{\prime}$ is started with the evaluation of the product of all 6 even spin structures, $\vartheta\left[\delta_{2}^{+}\right] \vartheta\left[\delta_{2}^{-}\right] \vartheta\left[\delta_{3}^{+}\right] \vartheta\left[\delta_{3}^{-}\right] \vartheta\left[\delta_{4}^{+}\right] \vartheta\left[\delta_{4}^{-}\right]$. This object is independent of the remaining choices of $c, d, e, f$, since a permutation of these objects simply permutes the various factors in the product. To evaluate it, we choose $(c, d, e, f)=(1,3,5,6)$. The even spin structures are then determined by (B.7) (see also (2.11)), and the $\vartheta$-constants are expressed in normalized form by

$$
\begin{aligned}
& \vartheta\left[\delta_{2}^{+}\right]=\vartheta\left[\delta_{7}+2 \nu_{d}\right]=-\vartheta\left[\delta_{7}\right] \\
& \vartheta\left[\delta_{2}^{-}\right]=\vartheta\left[\delta_{8}+2 \nu_{d}\right]=-\vartheta\left[\delta_{8}\right] \\
& \vartheta\left[\delta_{3}^{+}\right]=\vartheta\left[\delta_{1}+2 \delta_{0}\right]=+\vartheta\left[\delta_{1}\right] \\
& \vartheta\left[\delta_{3}^{-}\right]=\vartheta\left[\delta_{2}+2 \delta_{0}\right]=+\vartheta\left[\delta_{2}\right] \\
& \vartheta\left[\delta_{4}^{+}\right]=\vartheta\left[\delta_{3}+2 \nu_{e}\right]=+\vartheta\left[\delta_{3}\right] \\
& \vartheta\left[\delta_{4}^{-}\right]=\vartheta\left[\delta_{4}+2 \nu_{e}\right]=+\vartheta\left[\delta_{4}\right]
\end{aligned}
$$

Therefore, given the choice for $\varepsilon=-\nu_{2}+\nu_{4}$, the following product is the same for any choices of $c, d, e, f \neq 2,4$, and we have

$$
\vartheta\left[\delta_{2}^{+}\right] \vartheta\left[\delta_{2}^{-}\right] \vartheta\left[\delta_{3}^{+}\right] \vartheta\left[\delta_{3}^{-}\right] \vartheta\left[\delta_{4}^{+}\right] \vartheta\left[\delta_{4}^{-}\right]=+\vartheta\left[\delta_{1}\right] \vartheta\left[\delta_{2}\right] \vartheta\left[\delta_{3}\right] \vartheta\left[\delta_{4}\right] \vartheta\left[\delta_{7}\right] \vartheta\left[\delta_{8}\right]
$$

Next, the expressions for $\mathcal{M}_{a b}, a, b=1, \cdots, 6$ are needed. As was shown in [4], these objects may be expressed as products of $\vartheta$-constants, times a non-intrinsic sign factor. Normalizing the $\vartheta$-constants on the even spin structures in canonical form, as given by (B.5), the sign factors $m_{a b}$ are tabulated in (B.24). The quantities needed are given by

$$
\begin{aligned}
\mathcal{M}_{24} & =-\pi^{2} \vartheta\left[\delta_{0}\right] \vartheta\left[\delta_{5}\right] \vartheta\left[\delta_{6}\right] \vartheta\left[\delta_{9}\right] \\
\mathcal{M}_{2 c} \mathcal{M}_{c d} \mathcal{M}_{d 2} & =+m_{2 c} m_{c d} m_{d 2} \pi^{6} \vartheta[\delta]^{2} \prod_{i=0}^{9} \vartheta\left[\delta_{i}\right]
\end{aligned}
$$

Combining all of the above, we have

$$
\kappa^{\prime}=-m_{2 c} m_{c d} m_{d 2}
$$

Table 1 below summarizes the results of the case by case calculation of $\kappa^{\prime}$. 


\begin{tabular}{|c|c||c||c|c|c||c|}
\hline$c$ & $d$ & $\delta$ & $m_{2 c}$ & $m_{c d}$ & $m_{d 2}$ & $\kappa^{\prime}$ \\
\hline \hline 1 & 3 & $\delta_{7}$ & + & - & - & - \\
\hline 1 & 5 & $\delta_{3}$ & + & + & - & + \\
\hline 1 & 6 & $\delta_{2}$ & + & - & - & - \\
\hline 3 & 5 & $\delta_{1}$ & + & + & - & + \\
\hline 3 & 6 & $\delta_{4}$ & + & - & - & - \\
\hline 5 & 6 & $\delta_{8}$ & + & - & - & - \\
\hline \hline
\end{tabular}

Table 1: Calculation of $\kappa^{\prime}$

\subsubsection{Calculating the sign of $\Gamma[\delta ; \varepsilon]$}

A general expression for $\kappa$ was obtained in (5.34). For our present purposes, with $a=$ $2, b=4$, this expression becomes,

$$
\kappa=\left\langle\nu_{c} \mid \nu_{d}\right\rangle \exp 4 \pi i\left(-\nu_{d}^{\prime} \nu_{2}^{\prime \prime}+\left(\nu_{c}^{\prime}+\nu_{d}^{\prime}\right) \nu_{f}^{\prime \prime}+\nu_{4}^{\prime} \nu_{c}^{\prime \prime}\right)
$$

Notice that $\kappa$ depends on $f$ as well as on $c$ and $d$. Thus, $\kappa$ does not just depend on $\varepsilon$ and $\delta$ (i.e. only on $\nu_{a}, \nu_{b}$ and $\nu_{c}+\nu_{d}$ ), but also on the choice of $\nu_{f}$ versus $\nu_{e}$. Furthermore, $\kappa$ is not, in general, symmetric under the interchange of $c$ and $d$; instead, we have

$$
\frac{\kappa(c, d)}{\kappa(d, c)}=-\left\langle\nu_{c} \mid \nu_{d}\right\rangle
$$

for fixed $a, b, e, f$. Fortunately, these non-intrinsic dependences of $\kappa$ are being compensated in the expression for $\Gamma[\delta ; \varepsilon]$ by the presence of another sign factor $\sigma\left(\mu_{i}, \mu_{j}\right)$. Recall that for given $i$, there can be two choices for $j$. These choices are actually correlated with the choices for $f$ in $\kappa$.

Clearly, $f$-dependence enters only when $\left(\nu_{c}^{\prime}+\nu_{d}^{\prime}\right) \nu_{f}^{\prime \prime} \neq 0$, which is the case (here with $a=2$ and $b=4)$ when $c=1, d=6$ and $c=3, d=5$. To identify the correlation between the choice of $f$ in $\kappa$ and that of $j$ in $\sigma\left(\mu_{i}, \mu_{j}\right)$, we need consider only these cases.

- $c=1, d=6$ implies

$$
\left\{\begin{array} { l } 
{ \delta _ { i } ^ { + } = \delta _ { 2 } } \\
{ \delta _ { i } ^ { - } = \delta _ { 1 } }
\end{array} \quad \left\{\begin{array}{l}
\delta_{j}^{+}=\nu_{1}+\nu_{4}+\nu_{f} \\
\delta_{j}^{-}=\nu_{1}+\nu_{2}+\nu_{f}
\end{array}\right.\right.
$$

For $f=3$, we have $\delta_{j}^{+}=\delta_{8}$ and therefore $j=2$. For $f=5$, we have $\delta_{j}^{+}=\delta_{4}$ and therefore $j=4$. 


\begin{tabular}{|c|c|c||c||c|c|c||c|c|c||c|}
\hline$c$ & $d$ & $f$ & $\delta$ & $\mu_{i}$ & $\mu_{j}$ & $\mu_{k}$ & $\kappa$ & $\kappa^{\prime}$ & $\sigma\left(\mu_{i}, \mu_{j}\right)$ & SIGN \\
\hline \hline 1 & 3 & 5 & $\delta_{7}$ & $\mu_{2}$ & $\mu_{4}$ & $\mu_{3}$ & + & - & + & - \\
& & 6 & & & $\mu_{3}$ & $\mu_{4}$ & + & - & + & \\
\hline 1 & 5 & 3 & $\delta_{3}$ & $\mu_{4}$ & $\mu_{2}$ & $\mu_{3}$ & + & + & - & - \\
& & 6 & & & $\mu_{3}$ & $\mu_{2}$ & + & + & - & \\
\hline 1 & 6 & 3 & $\delta_{2}$ & $\mu_{3}$ & $\mu_{2}$ & $\mu_{4}$ & - & - & - & - \\
& & 5 & & & $\mu_{4}$ & $\mu_{2}$ & + & - & + & \\
\hline 3 & 5 & 1 & $\delta_{1}$ & $\mu_{3}$ & $\mu_{2}$ & $\mu_{4}$ & - & + & - & + \\
& & 6 & & & $\mu_{4}$ & $\mu_{2}$ & + & + & + & \\
\hline 3 & 6 & 1 & $\delta_{4}$ & $\mu_{4}$ & $\mu_{2}$ & $\mu_{3}$ & + & - & - & + \\
& & 5 & & & $\mu_{3}$ & $\mu_{2}$ & + & - & - & \\
\hline 5 & 6 & 1 & $\delta_{8}$ & $\mu_{2}$ & $\mu_{4}$ & $\mu_{3}$ & - & - & + & + \\
& & 3 & & & $\mu_{3}$ & $\mu_{4}$ & - & - & + & \\
\hline \hline
\end{tabular}

Table 2: Calculation of the SIGN of $\Gamma[\delta ; \varepsilon]$

- $c=3, d=5$ implies

$$
\left\{\begin{array} { l } 
{ \delta _ { i } ^ { + } = \delta _ { 1 } } \\
{ \delta _ { i } ^ { - } = \delta _ { 2 } }
\end{array} \quad \left\{\begin{array}{l}
\delta_{j}^{+}=\nu_{3}+\nu_{4}+\nu_{f} \\
\delta_{j}^{-}=\nu_{2}+\nu_{3}+\nu_{f}
\end{array}\right.\right.
$$

For $f=1$, we have $\delta_{j}^{+}=\delta_{8}$ and therefore $j=2$. For $f=6$, we have $\delta_{j}^{+}=\delta_{3}$ and therefore $j=4$.

The corresponding results are summarized in Table 2 above.

Assembling all the results with the basic expression for $\Gamma[\delta ; \varepsilon]$, we obtain,

$$
\Gamma\left[\delta_{i}^{\sigma}, \varepsilon\right]=\sigma \frac{i}{8 \pi^{7}} \frac{\left\langle\nu_{0} \mid \mu_{i}\right\rangle}{\vartheta_{k}^{4}} \frac{\vartheta[\delta]^{2}}{\vartheta\left[\delta_{j}^{+}\right]^{2} \vartheta\left[\delta_{j}^{-}\right]^{2} \vartheta[\delta+\varepsilon]^{2}}
$$

Here, $(i, j, k)$ is a permutation of the genus 1 spin structure indices $(2,3,4)$, and $\delta=\delta_{i}^{\sigma}$ with $\sigma= \pm$. A more illuminating expression, whose form is more manifestly covariant is obtained by factoring out a combination of the $Z^{\mathrm{qu}}[\varepsilon]$ partition function for 4 twisted directions. Using the familiar genus 1 relation $\vartheta_{i} \vartheta_{j} \vartheta_{k}=2 \eta^{3}$, this leads to the following final expression,

$$
\Gamma\left[\delta_{i}^{\sigma}, \varepsilon\right]=\sigma\left\langle\nu_{0} \mid \mu_{i}\right\rangle \frac{i}{(2 \pi)^{7}} \frac{\vartheta_{i}^{4}}{\eta^{12}}\left(\frac{\vartheta\left[\delta_{i}^{\sigma}\right]^{2}}{\vartheta\left[\delta_{i}^{(-\sigma)}\right]^{2}}\right)\left(\frac{\vartheta_{j}^{4}}{\vartheta\left[\delta_{j}^{+}\right]^{2} \vartheta\left[\delta_{j}^{-}\right]^{2}}\right)
$$

which is independent of $j$. Here, $\sigma= \pm$ according to which spin structure $\delta_{i}^{ \pm}$is being evaluated. 


\section{Modular transformations and $\mathrm{Z}_{2}$ twisting}

The structure of the genus 2 modular group $S p(4, \mathbf{Z})$ and its action on spin structures, twists and $\vartheta$-functions is summarized in Appendix B.

\subsection{Subgroup preserving a given twist}

The subgroup of the modular group $S p(4, \mathbf{Z})$ which acts on spin structures or on half characteristics is $S p\left(4, \mathbf{Z}_{2}\right)$. It is isomorphic to the permutation group of the six branch points and therefore has $6 !=720$ elements. To determine the subgroup $H_{\varepsilon}$ of $S p\left(4, \mathbf{Z}_{2}\right)$ that leaves a given twist $\varepsilon$ invariant, we proceed as follows. First, make a definite choice for a twist, so that the invariance equation becomes

$$
M=\left(\begin{array}{cc}
A & B \\
C & D
\end{array}\right) \quad \varepsilon=\left(\begin{array}{l|l}
0 & 0 \\
0 & \frac{1}{2}
\end{array}\right) \quad\left(\begin{array}{c}
\varepsilon^{\prime} \\
\varepsilon^{\prime \prime}
\end{array}\right)=\left(\begin{array}{cc}
D & -C \\
-B & A
\end{array}\right)\left(\begin{array}{c}
\varepsilon^{\prime} \\
\varepsilon^{\prime \prime}
\end{array}\right)
$$

This equation puts the following restrictions on the entries $A_{12}=C_{12}=C_{22}=0$ and $A_{22}=1$ of the matrices. All elements of the group $H_{\varepsilon}$ are found by solving the symplectic relation $M J M^{T}=J$ under these restrictions. The independent generators for $H_{\varepsilon}$ are

$$
\text { generators of } H_{\varepsilon}=\left\{M_{1}, M_{2}, M_{3}, T_{2}=\Sigma T \Sigma, S_{2}=S M_{1} S M_{1}\right\}
$$

The full group may be parametrized in terms of the Abelian subgroup $M_{\varepsilon}$ of "translations",

$$
\begin{aligned}
H_{\varepsilon}^{0} & =\left\{I, M_{1}, M_{2}, M_{3}, M_{1} M_{2}, M_{1} M_{3}, M_{2} M_{3}, M_{1} M_{2} M_{3}\right\} \\
H_{\varepsilon} & =\left\{I, T_{2}, S_{2}, S_{2} T_{2}, S_{2}^{2}, S_{2}^{2} M_{1} T_{2}\right\} \times H_{\varepsilon}^{0}
\end{aligned}
$$

The matrices $M_{i}$ 's are given explicitly in Appendix B3. In total there are 48 elements. This number is as expected, since all elements of the group $S p\left(4, \mathbf{Z}_{2}\right)$ may be decomposed as the product of elements that move the twists from any given twist to any other twist times an element that preserves the twist. There are 15 non-trivial twists and hence $720=15 \times 48$. The transformation laws of the even spin structures under the generators of $H_{\varepsilon}$ are listed in Table 3.

\subsection{Transformations of the Schottky relations}

In (2.46), a Schottky relation is derived, but a sharper form (namely the precise sign involved in taking the square root of this relation) is needed in the present study, as indicated in (2.12). The square root may be fixed by checking the consistency of (2.12) with modular transformations and with degenerations, both of which are carried out in this subsection. 


\begin{tabular}{|c||c|c|c|c|c|c|c|c|c|c|}
\hline$\delta$ & $M_{1}$ & $M_{2}$ & $M_{3}$ & $T_{2}$ & $S_{2}$ & $\epsilon^{2}\left(M_{1}\right)$ & $\epsilon^{2}\left(M_{2}\right)$ & $\epsilon^{2}\left(M_{3}\right)$ & $\epsilon^{2}\left(T_{2}\right)$ & $\epsilon^{2}\left(S_{2}\right)$ \\
\hline \hline$\delta_{1}$ & $\delta_{3}$ & $\delta_{2}$ & $\delta_{1}$ & $\delta_{1}$ & $\delta_{3}$ & 1 & 1 & 1 & 1 & $i$ \\
\hline$\delta_{2}$ & $\delta_{4}$ & $\delta_{1}$ & $\delta_{2}$ & $\delta_{2}$ & $\delta_{4}$ & 1 & 1 & 1 & 1 & $i$ \\
\hline$\delta_{3}$ & $\delta_{1}$ & $\delta_{4}$ & $\delta_{3}$ & $\delta_{4}$ & $\delta_{7}$ & 1 & 1 & 1 & 1 & 1 \\
\hline$\delta_{4}$ & $\delta_{2}$ & $\delta_{3}$ & $\delta_{4}$ & $\delta_{3}$ & $\delta_{8}$ & 1 & 1 & 1 & 1 & 1 \\
\hline$\delta_{5}$ & $\delta_{6}$ & $\delta_{5}$ & $\delta_{6}$ & $\delta_{9}$ & $\delta_{6}$ & 1 & $i$ & 1 & 1 & $i$ \\
\hline$\delta_{6}$ & $\delta_{5}$ & $\delta_{6}$ & $\delta_{5}$ & $\delta_{0}$ & $\delta_{9}$ & 1 & $i$ & 1 & 1 & 1 \\
\hline$\delta_{7}$ & $\delta_{7}$ & $\delta_{8}$ & $\delta_{8}$ & $\delta_{7}$ & $\delta_{1}$ & $i$ & 1 & 1 & 1 & $i$ \\
\hline$\delta_{8}$ & $\delta_{8}$ & $\delta_{7}$ & $\delta_{7}$ & $\delta_{8}$ & $\delta_{2}$ & $i$ & 1 & 1 & 1 & $i$ \\
\hline$\delta_{9}$ & $\delta_{9}$ & $\delta_{9}$ & $\delta_{0}$ & $\delta_{5}$ & $\delta_{5}$ & $i$ & $i$ & -1 & 1 & $i$ \\
\hline$\delta_{0}$ & $\delta_{0}$ & $\delta_{0}$ & $\delta_{9}$ & $\delta_{6}$ & $\delta_{0}$ & $i$ & $i$ & -1 & 1 & -1 \\
\hline
\end{tabular}

Table 3: The modular group $H_{\varepsilon}$ acting on even spin structures

The crucial ingredients in this calculation are the factors $\epsilon(\delta, M)^{2}$ when $M \in H_{\varepsilon}$, since they will determine the transformation rules for the genus $2 \vartheta$-constants. To compute them, we make use of the values for $\epsilon\left(\delta, M_{i}\right)^{2}, i=1,2,3, \epsilon(\delta, S)^{2}, \epsilon(\delta, T)^{2}$, and $\epsilon(\delta, \Sigma)^{2}$, given in (B.16), as well as the following cocycle rule, which may be derived from the definition of $\epsilon(\delta, M)$ in terms of the $\vartheta$-constants,

$$
\epsilon\left(\kappa, M M^{\prime}\right)=\epsilon\left(\kappa, M^{\prime}\right) \times \epsilon\left(M^{\prime} \kappa, M\right)
$$

The results are summarized in Table 3, and may be readily applied to the calculation of the pairwise products $\epsilon\left(\delta_{j}, M\right)^{2} \epsilon\left(\delta_{j+1}, M\right)^{2}$. The results are given in Table 4.

\begin{tabular}{|c||c|c|c|c|c|}
\hline$j$ & $M_{1}$ & $M_{2}$ & $M_{3}$ & $T^{\prime}$ & $S_{2}$ \\
\hline \hline 1 & 1 & 1 & 1 & 1 & -1 \\
\hline 3 & 1 & 1 & 1 & 1 & 1 \\
\hline 7 & -1 & 1 & 1 & 1 & -1 \\
\hline \hline
\end{tabular}

Table 4: The modular group $H_{\varepsilon}$ acting on pairs $\delta^{ \pm}$of even spin structures

For the choice of twist made here, (2.12) becomes,

$$
\frac{\vartheta_{4}^{4}}{\vartheta_{2}^{4}}=\frac{\vartheta^{2}\left[\delta_{3}\right] \vartheta^{2}\left[\delta_{4}\right]}{\vartheta^{2}\left[\delta_{7}\right] \vartheta^{2}\left[\delta_{8}\right]} \quad \frac{\vartheta_{2}^{4}}{\vartheta_{3}^{4}}=\frac{\vartheta^{2}\left[\delta_{7}\right] \vartheta^{2}\left[\delta_{8}\right]}{\vartheta^{2}\left[\delta_{1}\right] \vartheta^{2}\left[\delta_{2}\right]} \quad \frac{\vartheta_{3}^{4}}{\vartheta_{4}^{4}}=\frac{\vartheta^{2}\left[\delta_{1}\right] \vartheta^{2}\left[\delta_{2}\right]}{\vartheta^{2}\left[\delta_{3}\right] \vartheta^{2}\left[\delta_{4}\right]}
$$


By inspecting Table 3, it is manifest that the transformations $M_{2}, M_{3}$ and $T_{2}$ do not act on these pairs at all. It remains to consider only the actions of $M_{1}$ and $S_{2}$,

$$
\begin{aligned}
& M_{1}\left(\frac{\vartheta_{3}^{4}}{\vartheta_{4}^{4}}\right)=M_{1}\left(\frac{\vartheta^{2}\left[\delta_{1}\right] \vartheta^{2}\left[\delta_{2}\right]}{\vartheta^{2}\left[\delta_{3}\right] \vartheta^{2}\left[\delta_{4}\right]}\right)=+\frac{\vartheta^{2}\left[\delta_{3}\right] \vartheta^{2}\left[\delta_{4}\right]}{\vartheta^{2}\left[\delta_{1}\right] \vartheta^{2}\left[\delta_{2}\right]}=+\frac{\vartheta_{4}^{4}}{\vartheta_{3}^{4}} \\
& M_{1}\left(\frac{\vartheta_{3}^{4}}{\vartheta_{2}^{4}}\right)=M_{1}\left(\frac{\vartheta^{2}\left[\delta_{1}\right] \vartheta^{2}\left[\delta_{2}\right]}{\vartheta^{2}\left[\delta_{7}\right] \vartheta^{2}\left[\delta_{8}\right]}\right)=-\frac{\vartheta^{2}\left[\delta_{3}\right] \vartheta^{2}\left[\delta_{4}\right]}{\vartheta^{2}\left[\delta_{7}\right] \vartheta^{2}\left[\delta_{8}\right]}=-\frac{\vartheta_{4}^{4}}{\vartheta_{2}^{4}} \\
& S_{2}\left(\frac{\vartheta_{3}^{4}}{\vartheta_{4}^{4}}\right)=S_{2}\left(\frac{\vartheta^{2}\left[\delta_{1}\right] \vartheta^{2}\left[\delta_{2}\right]}{\vartheta^{2}\left[\delta_{3}\right] \vartheta^{2}\left[\delta_{4}\right]}\right)=-\frac{\vartheta^{2}\left[\delta_{3}\right] \vartheta^{2}\left[\delta_{4}\right]}{\vartheta^{2}\left[\delta_{7}\right] \vartheta^{2}\left[\delta_{8}\right]}=-\frac{\vartheta_{4}^{4}}{\vartheta_{2}^{4}} \\
& S_{2}\left(\frac{\vartheta_{3}^{4}}{\vartheta_{2}^{4}}\right)=S_{2}\left(\frac{\vartheta^{2}\left[\delta_{1}\right] \vartheta^{2}\left[\delta_{2}\right]}{\vartheta^{2}\left[\delta_{7}\right] \vartheta^{2}\left[\delta_{8}\right]}\right)=+\frac{\vartheta^{2}\left[\delta_{3}\right] \vartheta^{2}\left[\delta_{4}\right]}{\vartheta^{2}\left[\delta_{1}\right] \vartheta^{2}\left[\delta_{2}\right]}=+\frac{\vartheta_{4}^{4}}{\vartheta_{3}^{4}}
\end{aligned}
$$

The transformation properties of $\vartheta$-constants for genus 1 , under the two canonical generators, denoted here by $T^{(1)}$ and $S^{(1)}$ are given by

$$
\left\{\begin{array}{lll}
T^{(1)} & : & \tau \rightarrow \tau+1 \\
S^{(1)} & : & \tau \rightarrow-1 / \tau
\end{array}\right.
$$

We have

$$
T^{(1)}\left\{\begin{array} { l } 
{ \vartheta _ { 2 } ( \tau + 1 ) = e ^ { i \pi / 4 } \vartheta _ { 2 } ( \tau ) } \\
{ \vartheta _ { 3 } ( \tau + 1 ) = \vartheta _ { 4 } ( \tau ) } \\
{ \vartheta _ { 4 } ( \tau + 1 ) = \vartheta _ { 3 } ( \tau ) }
\end{array} \quad S ^ { ( 1 ) } \left\{\begin{array}{l}
\vartheta_{2}(-1 / \tau)=\sqrt{-i \tau} \vartheta_{4}(\tau) \\
\vartheta_{3}(-1 / \tau)=\sqrt{-i \tau} \vartheta_{3}(\tau) \\
\vartheta_{4}(-1 / \tau)=\sqrt{-i \tau} \vartheta_{2}(\tau)
\end{array}\right.\right.
$$

Therefore, it is clear that, signs and all, we have

$$
\begin{array}{rll}
M_{1} & \longrightarrow & T^{(1)} \\
S_{2} & \longrightarrow & T^{(1)} S^{(1)}
\end{array}
$$

Therefore, the genus 2 modular transformations $M_{1}$ and $S_{2}$ indeed induce ordinary genus 1 modular transformations on the Prym period. As a result, all the modular transformations in $H_{\varepsilon}$ induce modular transformations on $\tau_{\varepsilon}$, which belong to the genus 1 modular group, according to the above correspondence.

\subsection{Modular orbits of the twists under $H_{\varepsilon}$}

First, a parametrization of the twists is needed,

$$
\begin{aligned}
& 2 \varepsilon_{1}=\left(\begin{array}{l|l}
0 & 0 \\
0 & 0
\end{array}\right) \quad 2 \varepsilon_{2}=\left(\begin{array}{l|l}
0 & 0 \\
0 & 1
\end{array}\right) \quad 2 \varepsilon_{3}=\left(\begin{array}{l|l}
0 & 1 \\
0 & 0
\end{array}\right) \quad 2 \varepsilon_{4}=\left(\begin{array}{l|l}
0 & 1 \\
0 & 1
\end{array}\right) \\
& 2 \varepsilon_{5}=\left(\begin{array}{l|l}
0 & 0 \\
1 & 0
\end{array}\right) \quad 2 \varepsilon_{6}=\left(\begin{array}{l|l}
0 & 1 \\
1 & 0
\end{array}\right) \quad 2 \varepsilon_{7}=\left(\begin{array}{l|l}
1 & 0 \\
0 & 0
\end{array}\right) \quad 2 \varepsilon_{8}=\left(\begin{array}{l|l}
1 & 0 \\
0 & 1
\end{array}\right) \\
& 2 \varepsilon_{9}=\left(\begin{array}{l|l}
1 & 0 \\
1 & 0
\end{array}\right) \quad 2 \varepsilon_{10}=\left(\begin{array}{l|l}
1 & 1 \\
1 & 1
\end{array}\right) \quad 2 \varepsilon_{11}=\left(\begin{array}{l|l}
0 & 0 \\
1 & 1
\end{array}\right) \quad 2 \varepsilon_{12}=\left(\begin{array}{l|l}
1 & 1 \\
0 & 0
\end{array}\right) \\
& 2 \varepsilon_{13}=\left(\begin{array}{l|l}
0 & 1 \\
1 & 1
\end{array}\right) \quad 2 \varepsilon_{14}=\left(\begin{array}{l|l}
1 & 1 \\
0 & 1
\end{array}\right) \quad 2 \varepsilon_{15}=\left(\begin{array}{l|l}
1 & 0 \\
1 & 1
\end{array}\right) \quad 2 \varepsilon_{16}=\left(\begin{array}{l|l}
1 & 1 \\
1 & 0
\end{array}\right)
\end{aligned}
$$


Notice that the standard twist adopted previously is given by $\varepsilon=\varepsilon_{2}$, and that $\varepsilon_{1}$ is no twist at all. The modular transformations under the subgroup $H_{\varepsilon}$ are then easily computed from the transformation formula for the twists.

\begin{tabular}{|c||c|c|c|c|c|c|c|c|c|c|c|c|c|c|c|c|}
\hline$M$ & $\varepsilon_{1}$ & $\varepsilon_{2}$ & $\varepsilon_{3}$ & $\varepsilon_{4}$ & $\varepsilon_{5}$ & $\varepsilon_{6}$ & $\varepsilon_{7}$ & $\varepsilon_{8}$ & $\varepsilon_{9}$ & $\varepsilon_{10}$ & $\varepsilon_{11}$ & $\varepsilon_{12}$ & $\varepsilon_{13}$ & $\varepsilon_{14}$ & $\varepsilon_{15}$ & $\varepsilon_{16}$ \\
\hline \hline$M_{1}$ & $\varepsilon_{1}$ & $\varepsilon_{2}$ & $\varepsilon_{3}$ & $\varepsilon_{4}$ & $\varepsilon_{5}$ & $\varepsilon_{6}$ & $\varepsilon_{12}$ & $\varepsilon_{14}$ & $\varepsilon_{16}$ & $\varepsilon_{15}$ & $\varepsilon_{11}$ & $\varepsilon_{7}$ & $\varepsilon_{13}$ & $\varepsilon_{8}$ & $\varepsilon_{10}$ & $\varepsilon_{9}$ \\
\hline$M_{2}$ & $\varepsilon_{1}$ & $\varepsilon_{2}$ & $\varepsilon_{3}$ & $\varepsilon_{4}$ & $\varepsilon_{11}$ & $\varepsilon_{13}$ & $\varepsilon_{7}$ & $\varepsilon_{8}$ & $\varepsilon_{15}$ & $\varepsilon_{16}$ & $\varepsilon_{5}$ & $\varepsilon_{12}$ & $\varepsilon_{6}$ & $\varepsilon_{14}$ & $\varepsilon_{9}$ & $\varepsilon_{10}$ \\
\hline$M_{3}$ & $\varepsilon_{1}$ & $\varepsilon_{2}$ & $\varepsilon_{3}$ & $\varepsilon_{4}$ & $\varepsilon_{6}$ & $\varepsilon_{5}$ & $\varepsilon_{8}$ & $\varepsilon_{7}$ & $\varepsilon_{10}$ & $\varepsilon_{9}$ & $\varepsilon_{13}$ & $\varepsilon_{14}$ & $\varepsilon_{11}$ & $\varepsilon_{12}$ & $\varepsilon_{16}$ & $\varepsilon_{15}$ \\
\hline$T_{2}$ & $\varepsilon_{1}$ & $\varepsilon_{2}$ & $\varepsilon_{4}$ & $\varepsilon_{3}$ & $\varepsilon_{9}$ & $\varepsilon_{10}$ & $\varepsilon_{7}$ & $\varepsilon_{8}$ & $\varepsilon_{5}$ & $\varepsilon_{6}$ & $\varepsilon_{15}$ & $\varepsilon_{14}$ & $\varepsilon_{16}$ & $\varepsilon_{12}$ & $\varepsilon_{11}$ & $\varepsilon_{13}$ \\
\hline$S_{2}$ & $\varepsilon_{1}$ & $\varepsilon_{2}$ & $\varepsilon_{12}$ & $\varepsilon_{14}$ & $\varepsilon_{5}$ & $\varepsilon_{16}$ & $\varepsilon_{3}$ & $\varepsilon_{4}$ & $\varepsilon_{6}$ & $\varepsilon_{15}$ & $\varepsilon_{11}$ & $\varepsilon_{7}$ & $\varepsilon_{10}$ & $\varepsilon_{8}$ & $\varepsilon_{13}$ & $\varepsilon_{9}$ \\
\hline \hline
\end{tabular}

Table 5: The modular group $H_{\varepsilon}$ acting on twists

Simple inspection of Table 5 reveals 4 orbits,

$$
\begin{aligned}
\mathcal{O}_{0}[\varepsilon] & =\left\{\varepsilon_{1}\right\} \\
\mathcal{O}_{\varepsilon}[\varepsilon] & =\left\{\varepsilon_{2}\right\} \\
\mathcal{O}_{+}[\varepsilon] & =\left\{\varepsilon_{3}, \varepsilon_{4}, \varepsilon_{7}, \varepsilon_{8}, \varepsilon_{12}, \varepsilon_{14}\right\} \\
\mathcal{O}_{-}[\varepsilon] & =\left\{\varepsilon_{5}, \varepsilon_{6}, \varepsilon_{9}, \varepsilon_{10}, \varepsilon_{11}, \varepsilon_{13}, \varepsilon_{15}, \varepsilon_{16}\right\}
\end{aligned}
$$

The non-trivial orbits $\mathcal{O}_{ \pm}[\varepsilon]$ may be characterized in a simple modular covariant manner,

$$
v \in \mathcal{O}_{ \pm}[\varepsilon] \quad \Leftrightarrow \quad\langle\varepsilon \mid v\rangle= \pm 1 \text { and } v \neq \varepsilon_{1}, \varepsilon_{2}
$$

\subsection{Subgroups leaving two twists invariant}

The first twist may be denoted $\varepsilon$ and chosen as in (6.1). The non-trivial cases arise when the second twist $\alpha$ belongs to either orbit $\mathcal{O}_{ \pm}[\varepsilon]$. In each orbit, any representative may be taken for the second twist $\alpha$. The stabilizer groups of two twists $\varepsilon$ and $\alpha$ will be denoted by $H_{\varepsilon, \alpha}$; their generators may be deduced from inspection of Table 5 , and given by

$$
\begin{array}{ll}
H_{\varepsilon, \varepsilon_{3}} \sim \text { generators } & \left\{M_{1}, M_{2}, M_{3}\right\} \\
H_{\varepsilon, \varepsilon_{5}} \sim \text { generators } & \left\{M_{1}, S_{2},\left(M_{3} S_{2} T_{2}\right)\right\}
\end{array}
$$

The group $H_{\varepsilon, \varepsilon_{3}}$ is Abelian, but the group $H_{\varepsilon, \varepsilon_{5}}$ is non-Abelian,

\section{Asymptotic behavior of the chiral measure}

Given the choice of homology basis adopted throughout this paper, the period matrix may be parametrized as follows,

$$
\Omega=\left(\begin{array}{cc}
\tau_{1} & \tau \\
\tau & \tau_{2}
\end{array}\right)
$$


where $\tau_{1,2}, \tau \in \mathbf{C}$, subject to the constraint $\operatorname{Im} \Omega>0$. Degenerations fall into two classes according to whether the degeneration separates the surface into two disconnected components or leaves the surface connected. Separating (resp. non-separating) degenerations result from the shrinking of a homologically trivial (resp. non-trivial) 1- cycle. The limit $\tau \rightarrow 0$ is separating, while the limits $\tau_{1} \rightarrow+i \infty$ or $\tau_{2} \rightarrow+i \infty$ are non-separating.

The flat space-time chiral measure depends on the spin structure and the limiting behavior of the measure therefore depends upon the inter-relation between the spin structure the homology of the degenerating cycle. These degenerations were worked out explicitly - to leading order - in [4], section 8.

The $\mathbf{Z}_{2}$-twisted chiral measure (1.10) depends on the spin structure $\delta$, AND on the twist $\varepsilon$. Its limiting behavior will therefore depend upon the inter-relations between not only the spin structure and the homology of the degenerating cycle, but also upon the twist. Here, we shall derive the limit of only two representative cases, one separating, the other non-separating; the other cases are similar, but their number is simply too large to discuss usefully here. Finally, only the case where $n=4$ dimensions are $\mathbf{Z}_{2}$-twisted will be discussed, since this will be the case of greatest physical interest, as discussed in the next subsection.

\subsection{Separating degeneration}

This limit corresponds to letting $\tau \rightarrow 0$, while keeping $\tau_{1,2}$ fixed. The leading behavior was given in [4]; the leading and subleading behaviors are as follows,

$$
\begin{aligned}
& \vartheta\left[\begin{array}{l}
\mu_{i} \\
\mu_{j}
\end{array}\right](0, \Omega)=\vartheta_{i}\left(0, \tau_{1}\right) \vartheta_{j}\left(0, \tau_{2}\right)\left\{1+2 \tau^{2} \partial \ln \vartheta_{i}\left(0, \tau_{1}\right) \partial \ln \vartheta_{j}\left(0, \tau_{2}\right)\right\} \\
& \Xi_{6}\left[\begin{array}{l}
\mu_{i} \\
\mu_{j}
\end{array}\right](\Omega)=2^{8}\left\langle\mu_{i} \mid \nu_{0}\right\rangle\left\langle\mu_{j} \mid \nu_{0}\right\rangle \eta\left(\tau_{1}\right)^{12} \eta\left(\tau_{2}\right)^{12}\left\{-1+\frac{\tau^{2}}{2}\left[3 \partial \ln \vartheta_{i}^{4}\left(0, \tau_{1}\right) \partial \ln \vartheta_{j}^{4}\left(0, \tau_{2}\right)\right.\right. \\
& \left.\left.-\partial \ln \eta\left(\tau_{1}\right)^{12} \partial \ln \vartheta_{j}^{4}\left(0, \tau_{2}\right)-\partial \ln \eta\left(\tau_{2}\right)^{12} \partial \ln \vartheta_{i}^{4}\left(0, \tau_{1}\right)\right]\right\} \\
& \Psi_{10}(\Omega)=2^{12}(2 \pi \tau)^{2} \eta\left(\tau_{1}\right)^{24} \eta\left(\tau_{2}\right)^{24}\left\{1+48 \tau^{2} \partial \ln \eta\left(\tau_{1}\right) \partial \ln \eta\left(\tau_{2}\right)\right\}
\end{aligned}
$$

Here, $\mu_{i}$ and $\mu_{j}$ represent the even spin structures on each genus 1 component; the even spin structure which restricts to the odd spin structure on each genus 1 component will not be needed here.

First, the limiting behavior is needed for the Prym period, since it enters into the form of the chiral measure. Using the Schottky relation (1.6) as well as the above limiting behaviors, we find

$$
\tau_{\varepsilon}=\tau_{1}+\tau^{2} \partial \ln \left(\vartheta_{3}\left(0, \tau_{2}\right) \vartheta_{4}\left(0, \tau_{2}\right)\right)+\mathcal{O}\left(\tau^{4}\right)
$$


up to shifts $\tau_{\varepsilon} \rightarrow \tau_{\varepsilon}+4$. Second, we need the bosonic twisted partition function factor

$$
\frac{\vartheta\left[\delta_{j}^{+}\right](0, \Omega)^{2} \vartheta\left[\delta_{j}^{-}\right](0, \Omega)^{2}}{\vartheta_{j}\left(0, \tau_{\varepsilon}\right)^{4}}=\vartheta_{3}\left(0, \tau_{2}\right)^{2} \vartheta_{4}\left(0, \tau_{2}\right)^{2}+\mathcal{O}\left(\tau^{4}\right)
$$

which is clearly independent of $j$ to this order. Third, the limit of the terms involving $\Gamma[\delta ; \varepsilon]$ is needed. When $n=4$, as is being assumed here, it is advantageous to consider directly the quantity

$$
\frac{Z_{C}\left[\delta_{i}^{\sigma}, \varepsilon\right]}{Z_{M}\left[\delta_{i}^{\sigma}\right]} \Gamma\left[\delta_{i}^{\sigma}, \varepsilon\right]=\left\langle\nu_{0} \mid \mu_{i}\right\rangle \frac{i \sigma}{(2 \pi)^{7}} \frac{\vartheta_{i}\left(0, \tau_{\varepsilon}\right)^{4}}{\eta\left(\tau_{\varepsilon}\right)^{12}} \quad i=2,3,4, \sigma= \pm
$$

In view of the relation between $\tau_{1}$ and $\tau_{\varepsilon}$, the limit as $\tau \rightarrow 0$ of this quantity is regular and is obtained simply from the above formula by replacing $\tau_{\varepsilon}$ by $\tau_{1}$. Putting all together, we find

$$
\begin{aligned}
d \mu_{C}\left[\delta_{i}^{\sigma}, \varepsilon\right]\left(p_{\varepsilon}^{\mu}\right)= & e^{i \pi \tau_{\varepsilon} p_{\varepsilon}^{2}} \frac{\left\langle\nu_{0} \mid \mu_{i}\right\rangle}{2^{6} \pi^{8}} \frac{\vartheta_{i}\left(0, \tau_{1}\right)^{4}}{\eta\left(\tau_{1}\right)^{12}} \\
& \times\left\{\frac{-\sigma}{\tau^{2} \vartheta_{2}\left(0, \tau_{2}\right)^{4}}-2 \pi i\left(\sigma+\frac{1}{2}\right) \partial \ln \vartheta_{i}\left(0, \tau_{1}\right)-\sigma \frac{\pi^{2}}{2} p_{\varepsilon}^{2}\right\}
\end{aligned}
$$

The physical analysis of this limit is as follows.

The $1 / \tau^{2}$ singularity, which is familiar from the flat space-time chiral measure, produces tachyon and massless scalar singularities in the channel connecting both genus 1 components. This is as expected, since the six spin structures $\delta_{i}^{\sigma}$ restrict to even spin structures on each genus 1 component. The summation over $\sigma$ corresponds to the GSO projection imposed on states in the NS sector which are traversing the $A_{2}$ cycle. This part of the GSO projection eliminates the corresponding tachyon intermediate state, and indeed the partially summed measure is regular, as may be seen from

$$
d \mu_{C}\left[\delta_{i}^{+}, \varepsilon\right]\left(p_{\varepsilon}^{\mu}\right)+d \mu_{C}\left[\delta_{i}^{-}, \varepsilon\right]\left(p_{\varepsilon}^{\mu}\right)=-i e^{i \pi \tau_{\varepsilon} p_{\varepsilon}^{2}} \frac{\left\langle\nu_{0} \mid \mu_{i}\right\rangle}{2^{5} \pi^{7}} \frac{\vartheta_{i}\left(0, \tau_{1}\right)^{4}}{\eta\left(\tau_{1}\right)^{12}} \partial \ln \vartheta_{i}\left(0, \tau_{1}\right)
$$

Notice also that a partial GSO resummation over the spin structures of the first genus 1 component vanishes to this order,

$$
\sum_{i=2,3,4} d \mu_{C}\left[\delta_{i}^{\sigma}, \varepsilon\right]\left(p_{\varepsilon}^{\mu}\right)=0
$$

in view of the Riemann identities for genus 1 ,

$$
\sum_{i=2,3,4}\left\langle\nu_{0} \mid \mu_{i}\right\rangle \vartheta_{i}\left(0, \tau_{1}\right)^{4}=\sum_{i=2,3,4}\left\langle\nu_{0} \mid \mu_{i}\right\rangle \vartheta_{i}\left(0, \tau_{1}\right)^{4} \partial \ln \vartheta_{i}\left(0, \tau_{1}\right)=0
$$

Again, this is as expected in view of space-time supersymmetry. 


\subsection{Non-separating Degenerations}

As mentioned in the opening paragraph to this subsection, many cases need to be distinguished based on the inter-relation of the spin structure, the twist and the shrinking cycle. The case considered here corresponds to letting the cycle $B_{2}$ grow to infinite length; more specifically, letting $\tau_{2} \rightarrow+i \infty$ while keeping $\tau$ and $\tau_{1}$ fixed. As usual, we introduce the variable $q \equiv \exp \left\{i \pi \tau_{2}\right\}$, in terms of which the limit is given by $q \rightarrow 0$. We recall from [4] the following limits,

$$
\vartheta\left[\begin{array}{l}
\mu_{i} \\
\mu_{j}
\end{array}\right](0, \Omega)=\vartheta_{i}\left(0, \tau_{1}\right)+\mathcal{O}(q), \quad \mu_{j}=(00),\left(0 \frac{1}{2}\right)
$$

As a result, the Schottky relation giving $\tau_{\varepsilon}$ in terms of the period matrix $\Omega+I J$ simply yield $\tau_{\varepsilon}=\tau_{1}$ up to shifts in $4 \mathbf{Z}$. The ratio $Z_{C} / Z_{M} \rightarrow 1$ in this limit. The following combination,

$$
\frac{Z_{C}\left[\delta_{i}^{\sigma}, \varepsilon\right]}{Z_{M}\left[\delta_{i}^{\sigma}\right]} \Gamma\left[\delta_{i}^{\sigma}, \varepsilon\right] \rightarrow\left\langle\nu_{0} \mid \mu_{i}\right\rangle \frac{i \sigma}{(2 \pi)^{7}} \frac{\vartheta_{i}\left(0, \tau_{1}\right)^{4}}{\eta\left(\tau_{1}\right)^{12}}
$$

has a smooth limit. Putting all together, we obtain the following limits for the full measure,

$$
d \mu_{C}\left[\delta_{i}^{\sigma} ; \varepsilon\right]\left(p_{\varepsilon}\right)=e^{i \pi \tau_{\varepsilon} p_{\varepsilon}^{2}} \frac{\sigma}{q} \frac{\left\langle\nu_{0} \mid \mu_{i}\right\rangle \vartheta_{i}\left(\frac{\tau}{2}, \tau_{1}\right)^{4}+\vartheta_{1}\left(\frac{\tau}{2}, \tau_{1}\right)^{4}}{2^{8} \pi^{6} \eta\left(\tau_{1}\right)^{6} \vartheta_{1}\left(\tau, \tau_{1}\right)^{2}}+\mathcal{O}(1)
$$

This result coincides with the untwisted case, as expected.

\section{Applications to Physical Theories}

In this section, specific physical superstring theories are considered that are constructed from flat Minkowski space-time orbifolded by groups acting by reflections and shift. When the action of the orbifold group is the same (resp. different) on left and right movers, the orbifold is referred to as symmetric (resp. asymmetric). The models of [9], for example, are asymmetric orbifolds of Type II superstring theory. For definiteness, the present study will concentrate on Type II theories with six or fewer compactified dimensions, but the methods may be extended to heterotic orbifolds and/or more compactified dimensions.

The construction of symmetric orbifolds may be carried out directly from the functional integral by inserting projection operators and including twisted sectors; for completeness, it will be briefly reviewed below. The construction of asymmetric orbifolds, on the other hand cannot, in general, be carried out directly from the functional integral. Therefore, properties that are known to hold generally for symmetric orbifolds (such as their behavior under modular transformations) may or may not hold for asymmetric orbifolds. Various

methods have been proposed to circumvent these obstacles. The method of [40] uses a 
doubling of the left- and right moving degrees of freedom, while the results of [41] rather suggest the use of operator methods. (For reviews and further references, see e.g. [42].)

The approach taken here to the construction of asymmetric orbifold superstring theories will be based on chiral splitting. The starting point will be the construction of the chiral blocks, carried out separately for the left-movers and for the right-movers. Once the chiral blocks are in hand, string amplitudes are obtained by assembling these left and right chiral blocks in a manner consistent with the definition of the asymmetric orbifold model as well as with modular invariance. This approach will be discussed in enough detail here so that the cosmological constant in the KKS models can be investigated. Further study will appear in a forthcoming publication [24].

\subsection{Chiral splitting of symmetric orbifolds}

Let $G$ be an orbifold group acting on $\mathbf{R}^{n}$, with $n \leq 6$, i.e. with at least four uncompactified space-time dimensions. An element $g \in G$ is an Euclidean transformation on $\mathbf{R}^{n}$, consisting of a rotation $R_{g} \in O(n)$ and a shift $v_{g} \in \mathbf{R}^{n}$, so that $g=\left(R_{g}, v_{g}\right)$, possibly supplemented by an action on internal quantum numbers. The action on the fields is given by $g x=R_{g} x+v_{g}$ and $g \psi_{ \pm}=R_{g} \psi_{ \pm}$, and thus symmetric on left- and right movers. Clearly, the non-trivial action of $G$ on the fermion fields and on the momentum and oscillator modes of the bosonic fields is only by the subgroup of rotations $\left(R_{g}, 0\right)$ (obtained by simply omitting the shift $v_{g}$ for each $g \in G$ ), which form the point group $P_{G}$. The subgroup of elements of the form $(I, v)$ on the other hand only acts on the zero modes of $x$ and forms a lattice $\Lambda_{G}$; the coset $T_{G}=\mathbf{R}^{n} / \Lambda_{G}$ is a torus. The symmetric orbifold may be constructed in two equivalent ways;

$$
\mathbf{R}^{n} / G=T_{G} / \bar{P}_{G}
$$

i.e. as the coset of $\mathbf{R}^{n}$ by the full orbifold group $G$, or as the coset of the torus $T_{G}$ by the group $\bar{P}_{G}=G / \Lambda_{G}$, which is isomorphic to $P_{G}$. The construction via $T_{G} / \bar{P}_{G}$ is generally more convenient (because the groups $\bar{P}_{G}$ are usually finite, while $G$ is always infinite) and is better suited for dealing with asymmetric orbifolds; therefore it will be adopted throughout. The orbifold is Abelian (resp. non-Abelian) if the point group $P_{G} \sim \bar{P}_{G}$ is Abelian (resp. non-Abelian).

The orbifold string theory is constructed as follows. Let $A_{I}, B_{I}, I=1,2$ be a canonical homology basis and define the fermion fields $\psi_{ \pm}^{\mu}$ with a spin structure $\delta$. To the basis cycles $\left(A_{I}, B_{I}\right)$ we associate a sector $\left(a_{I}, b_{I}\right)$ of elements of $\bar{P}_{G}$ which satisfies the homotopy relation $\prod_{I=1,2} a_{I} b_{I} a_{I}^{-1} b_{I}^{-1}=I$. (For Abelian orbifolds, this condition is automatically fulfilled.) The sector is untwisted if $a_{I}$ and $b_{I}$ equal the identity in $\bar{P}_{G}$, and is twisted otherwise. In a given sector $\lambda=\left(a_{I}, b_{I}\right)$, the fields obey the following monodromy 
conditions, ${ }^{11}$

$$
\begin{aligned}
& \left(x, \psi_{ \pm}\right)\left(z+A_{I}\right)=\left(a_{I} x(z),(-1)^{2 \delta_{I}^{\prime}} a_{I} \psi_{ \pm}(z)\right) \\
& \left(x, \psi_{ \pm}\right)\left(z+B_{I}\right)=\left(b_{I} x(z),(-1)^{2 \delta_{I}^{\prime \prime}} b_{I} \psi_{ \pm}(z)\right)
\end{aligned}
$$

The string amplitude in the sector $\lambda$ is given by the familiar functional integral but where the fields now obey the monodromy conditions (8.2),

$$
\mathbf{A}_{C}[\delta ; \lambda]=\int D E_{M}^{A} D \Omega_{M} \delta(T) \int_{\left(a_{I}, b_{I}\right)} D X^{\mu} e^{-I_{m}}
$$

The same procedure of gauge-fixing and chiral splitting as was carried out in (3.12) may be applied here, and results in a set of chiral blocks.

The chiral blocks are indexed by the sector labels $\lambda=\left(a_{I}, b_{I}\right)$ and by the internal momenta associated respectively with uncompactified $\left(p_{u}\right)$ and compactified $\left(p_{\lambda}\right)$ dimensions. The effects due to the uncompactified internal momenta $p_{u}$ are the same as in flat spacetime and yield the familiar measure factor $(\operatorname{det} \operatorname{Im} \Omega)^{-5+n / 2}$; henceforth, the dependence on $p_{u}$ will suppressed. The internal momenta $p_{\lambda}$ associated with the compactified directions are discrete and will take values in the lattice $\Lambda_{G}^{*}$ dual to $\Lambda_{G}$. The chiral blocks will be denoted by $d \mu_{C}[\delta ; \lambda]\left(p_{\lambda}\right)=d \mu_{C}\left[\delta ; a_{I}, b_{I}\right]\left(p_{\lambda}\right)$ and the amplitude $\mathbf{A}_{C}$ take on the form,

$$
\mathbf{A}_{C}[\delta ; \lambda]=\int_{\mathcal{M}_{2}}(\operatorname{det} \operatorname{Im} \Omega)^{-5+n / 2} \sum_{p_{\lambda} \in \Lambda_{G}^{*}}\left|d \mu_{C}[\delta ; \lambda]\left(p_{\lambda}\right)\right|^{2}
$$

The physical problem with this amplitude is the presence of the tachyon, which in particular causes the integration over moduli space to diverge.

In the GSO projection, left and right fermions are treated independently (via chiral splitting, see $[20,21]$ ), and assigned spin structures $\delta_{L}$ and $\delta_{R}$ which are summed over independently. A consistent GSO projection will systematically eliminate the tachyon in all superstring loops. Isolating the chiral blocks introduces a phase arbitrariness. Thus, the GSO projection requires a consistent set of phases $\eta_{L}\left[\delta_{L} ; \lambda\right]$ and $\eta_{R}\left[\delta_{R} ; \lambda\right]$, which may depend upon the sector $\lambda$. The GSO projected amplitude for the symmetric orbifold is,

$$
Z_{G}=\int_{\mathcal{M}_{2}}(\operatorname{det} \operatorname{Im} \Omega)^{-5+\frac{n}{2}} \sum_{\lambda} \sum_{p_{\lambda} \in \Lambda_{G}^{*}} d \mu_{L}[\lambda]\left(p_{\lambda}\right) \wedge \overline{d \mu_{R}[\lambda]\left(p_{\lambda}\right)}
$$

where the GSO resummed chiral blocks are given by

$$
\begin{aligned}
d \mu_{L}[\lambda]\left(p_{\lambda}\right) & =\sum_{\delta_{L}} \eta_{L}\left[\delta_{L} ; \lambda\right] d \mu_{C}\left[\delta_{L} ; \lambda\right]\left(p_{\lambda}\right) \\
\overline{d \mu_{R}[\lambda]\left(p_{\lambda}\right)} & =\sum_{\delta_{R}} \eta_{R}\left[\delta_{R} ; \lambda\right] \overline{d \mu_{C}\left[\delta_{R} ; \lambda\right]\left(p_{\lambda}\right)}
\end{aligned}
$$

\footnotetext{
${ }^{11}$ In the fermion monodromy, an extra sign may arise depending on the reference spin structure chosen in the given homology basis. For simplicity, this factor has been omitted here.
} 
As is familiar from flat space-time, the phase assignments on the left and right GSO summation may be taken to be the same (as in Type IIA) or different (as in Type IIB) from one another, even though for symmetric orbifolds the chiral blocks for fixed spin structure for right movers are simply the complex conjugate of those for left movers.

\subsection{Asymmetric Orbifolds}

An asymmetric orbifold results from taking the coset of Minkowski space-time by a group $G$ that acts asymmetrically on left and right moving degrees of freedom. More precisely, an asymmetric orbifold group $G$ consists of pairs of Euclidean transformations of $\mathbf{R}^{n}$ $g=\left(R_{g L}, v_{g L} ; R_{g R}, v_{g R}\right)$. On the genuine conformal fields $\partial x$ and $\psi_{ \pm}$, propagating say on a cylinder, the action of the asymmetric orbifold group elements is clearly given by the point group $P_{G}$

$$
\begin{aligned}
& g\left(\partial_{z} x, \psi_{+}\right)=\left(R_{g L} \partial_{z} x, R_{g L} \psi_{+}\right) \\
& g\left(\partial_{\bar{z}} x, \psi_{-}\right)=\left(R_{g R} \partial_{\bar{z}} x, R_{g R} \psi_{-}\right)
\end{aligned}
$$

where $x$ is viewed as a field taking values in a torus $T_{G}$. The action by the shifts on the zero mode part of $x$, however, is more subtle; we shall not make use of it here and postpone a more detailed discussion to [24].

For a higher genus surface, the group elements $a_{I}, b_{I}$ assigned to the canonical homology cycles $A_{I}, B_{I}$ now also each come in a pair of left and right rotations, $a_{I}=\left(a_{L I} ; a_{R I}\right)$ and $b_{I}=\left(b_{L I} ; b_{R I}\right)$. One would naturally be led to consider fields obeying the monodromy conditions

$$
\begin{aligned}
& \left(\partial_{z} x, \psi_{+}\right)\left(z+A_{I}\right)=\left(a_{L I} \partial_{z} x(z),(-1)^{2 \delta_{I}^{\prime}} a_{L I} \psi_{+}(z)\right) \\
& \left(\partial_{z} x, \psi_{+}\right)\left(z+B_{I}\right)=\left(b_{L I} \partial_{z} x(z),(-1)^{2 \delta_{I}^{\prime \prime}} b_{L I} \psi_{+}(z)\right) \\
& \left(\partial_{\bar{z}} x, \psi_{-}\right)\left(z+A_{I}\right)=\left(a_{R I} \partial_{\bar{z}} x(z),(-1)^{2 \bar{\delta}_{I}^{\prime}} a_{R I} \psi_{-}(z)\right) \\
& \left(\partial_{\bar{z}} x, \psi_{-}\right)\left(z+B_{I}\right)=\left(b_{R I} \partial_{\bar{z}} x(z),(-1)^{2 \bar{\delta}_{I}^{\prime \prime}} b_{R I} \psi_{-}(z)\right)
\end{aligned}
$$

These monodromy conditions are, however, only formal, as no real field $x$ in the functional integral will exist that satisfies conditions with $a_{L I} \neq a_{R I}$ or $b_{L I} \neq b_{R I}$.

The strategy adopted here is to obtain the amplitudes to higher loop order for the asymmetric theory from the chiral blocks of symmetric orbifold theories. Specifically, each element $f \in P_{G}$ is written as a pair $f=\left(f_{L} ; f_{R}\right)$ of rotations of $\mathbf{R}^{n}$. The set of all elements $f_{L}$ forms a group, which will be denoted $P_{L}$, while the set of all elements $f_{R}$ forms a group $P_{R}$. The group $P_{G}$ is thus viewed as a subgroup of $P_{L} \otimes P_{R}$ subject to the pairing relation $f=\left(f_{L} ; f_{R}\right)$. The chiral blocks for the left movers are the holomorphic blocks of the symmetric theory with orbifold group $P_{L}$, while the chiral blocks for the right movers are the anti-holomorphic blocks of the symmetric theory with orbifold group $P_{R}$. The internal 
momenta of the left and right movers do not need to match, as is familiar from toroidal and orbifold compactifications of the heterotic string. They will be denoted by $p_{L}$ and $p_{R}$; the pair $\left(p_{L}, p_{R}\right)$ belongs to a self-dual even lattice associated with $G$. When the elements of $\bar{P}_{G}$ have non-trivial shifts, the self-dual lattice will, in general, depend on the sector label $\lambda=\left(a_{I}, b_{I}\right)$.

Thus, the block associated with the spin structures $\delta_{L}$ and $\delta_{R}$ and sector $\lambda=\left(a_{I}, b_{I}\right)$, where $a_{I}=\left(a_{L I} ; a_{R I}\right)$ and $b_{I}=\left(b_{L I} ; b_{R I}\right)$, and chiral sector labels $\lambda_{L}=\left(a_{L I}, b_{L I}\right), \lambda_{R}=$ $\left(a_{R I}, b_{R I}\right)$, is given by

$$
d \mu_{C}\left[\delta_{L} ; \lambda_{L}\right]\left(p_{L}\right) \wedge \overline{d \mu_{C}\left[\delta_{R} ; \lambda_{R}\right]\left(p_{R}\right)}
$$

Just as in the case of flat Minkowski space-time (or, as explained in the previous subsection, for symmetric orbifold compactifications) a GSO projection must be performed to eliminate the tachyon. For most interesting asymmetric orbifolds, this projection is also carried out in a chiral manner, i.e. summing independently over the spin structures of left and right fermions. The relevant GSO resummed blocks are then precisely those of (8.6) but now with different sector labels for left and right,

$$
\begin{aligned}
d \mu_{L}\left[\lambda_{L}\right]\left(p_{L}\right) & =\sum_{\delta_{L}} \eta_{L}\left[\delta_{L} ; \lambda_{L}\right] d \mu_{C}\left[\delta_{L} ; \lambda_{L}\right]\left(p_{L}\right) \\
\overline{d \mu_{R}\left[\lambda_{R}\right]\left(p_{R}\right)} & =\sum_{\delta_{R}} \eta_{R}\left[\delta_{R} ; \lambda_{R}\right] \overline{d \mu_{C}\left[\delta_{R} ; \lambda_{R}\right]\left(p_{R}\right)}
\end{aligned}
$$

In the next subsection, constraints will be established on the phases $\eta_{L}$ and $\eta_{R}$ arising from modular symmetry.

The proposal made here for the partition function $Z_{G}$ of the asymmetric orbifold theory with asymmetric orbifold group $G$ is given by the expression

$$
Z_{G}=\int_{\mathcal{M}_{2}}(\operatorname{det} \operatorname{Im} \Omega)^{-5+\frac{n}{2}} \sum_{p_{L}, p_{R}} \sum_{\lambda_{L}, \lambda_{R}} K\left(\lambda_{L}, \lambda_{R} ; p_{L}, p_{R}\right) d \mu_{L}\left[\lambda_{L}\right]\left(p_{L}\right) \wedge \overline{d \mu_{R}\left[\lambda_{R}\right]\left(p_{R}\right)}
$$

Here, the coefficients $K\left(\lambda_{L}, \lambda_{R}, p_{L}, p_{R}\right)$ may depend on both sets of left and right group elements and internal momenta. Non-trivial internal momentum dependence in $K$ will arise in particular when the group elements of $G$ involve non-trivial shifts. Clearly, much of the structure of the original asymmetric group $G$ is encoded in these coefficients. Their evaluation can be a subtle issue, even to one-loop, and we shall return to this in [24]. In this paper, we shall restrict attention to the pointwise vanishing of the cosmological constant, and examine only the vanishing of each GSO resummed chiral block $d \mu_{L}\left[\lambda_{L}\right]\left(p_{L}\right)$ and $\overline{d \mu_{R}\left[\lambda_{R}\right]\left(p_{R}\right)}$. 


\subsection{GSO projection phases and $\mathrm{Z}_{2}$-twisting}

In the models to be studied below, the point group $P_{G}$ will be Abelian and generated by chiral $\mathbf{Z}_{2}$ reflections (possibly together with an action on internal quantum numbers, such as by the operators $(-)^{F}$ ). In all such cases, the chiral blocks will be given (up to phases) by the $\mathbf{Z}_{2}$-twisted blocks $d \mu_{C}[\delta ; \varepsilon]\left(p_{\varepsilon}\right)$ which were derived earlier. The sector labels will be abbreviated by $\lambda \equiv\left(a_{I}, b_{I}\right)$. The twist $\varepsilon$, which enters the chiral blocks $d \mu_{C}[\delta ; \varepsilon]\left(p_{\varepsilon}\right)$ will be determined by the sector labels $\lambda$, whence the notation $\varepsilon=\varepsilon(\lambda)$.

It is assumed that the GSO summation over $\delta$ is carried out independently on leftand right-movers. For the models of greatest physical interest, the number of $\mathbf{Z}_{2}$-twisted dimensions is 4 . This is the number of twisted dimensions in the KKS model, as well as in the orbifolding of the Type II theories by a pure $Z_{2}$ twist which yield again the same Type II theory. Henceforth, the number of twisted dimensions will be assumed to be 4 .

In view of the above assumptions, the relevant summation is a chiral summation over the measure calculated in (5.11) with $n=4$. The result is given by (we use the abbreviation $\delta=\delta_{L}, \lambda=\lambda_{L}$ and $\left.p=p_{L}\right)$

$$
d \mu_{L}[\lambda](p)=\sum_{\delta} \eta_{L}[\delta ; \lambda] d \mu_{C}[\delta, \varepsilon(\lambda)](p)=d \mu_{L}^{(1)}[\lambda](p)+d \mu_{L}^{(2)}[\lambda](p)
$$

where the partial measures are defined by (still using the notation $\varepsilon=\varepsilon(\lambda)$ )

$$
\begin{aligned}
d \mu_{L}^{(1)}[\lambda](p) & =\sum_{\delta} \eta_{L}[\delta ; \lambda] e^{i \pi \tau_{\varepsilon} p^{2}} \frac{Z_{C}[\delta, \varepsilon]}{Z_{M}[\delta]}\left(i \pi p^{2}-4 \partial_{\tau_{\varepsilon}} \ln \vartheta_{i}\left(0, \tau_{\varepsilon}\right)\right) \Gamma[\delta ; \varepsilon] \\
d \mu_{L}^{(2)}[\lambda](p) & =\sum_{\delta} \eta_{L}[\delta ; \lambda] e^{i \pi \tau_{\varepsilon} p^{2}} \frac{Z_{C}[\delta, \varepsilon]}{Z_{M}[\delta]} \frac{\Xi_{6}[\delta] \vartheta[\delta]^{4}}{16 \pi^{6} \Psi_{10}}
\end{aligned}
$$

Here, $\eta_{L}[\delta ; \lambda]$ are the left chiral GSO projection phases, which remain to be determined.

The assumption $n=4$ actually leads to considerable simplifications. A key ingredient in the chiral blocks is the ratio of chiral partition functions for the twisted space dimensions. For $n=4$, this quantity simplifies,

$$
\frac{Z_{C}[\delta ; \varepsilon]}{Z_{M}[\delta]}=\frac{\vartheta\left[\delta_{j}^{+}\right]^{2} \vartheta\left[\delta_{j}^{-}\right]^{2} \vartheta[\delta+\epsilon]^{2}}{\vartheta_{j}^{4} \vartheta[\delta]^{2}}
$$

since now only squares of $\vartheta$-constants are involved.

Next, the expression for $\Gamma[\delta ; \varepsilon]$ was calculated in (5.44). Finally, the Prym period $\tau_{\varepsilon}$ is determined by the Schottky relations in terms of the super-period matrix, which is now simply denoted by $\Omega_{I J}$. In our formalism, $\Omega_{I J}$ is interpreted as the period matrix of a bosonic spin structure independent Riemann surface. Therefore, the Prym period $\tau_{\varepsilon}$ must 
be viewed as independent of the spin structures $\delta$, and the Gaussian involving $\tau_{\varepsilon}$ may be factored out of the sum over $\delta$. The following simplified expressions are obtained,

$$
\begin{aligned}
d \mu_{L}^{(1)}[\lambda](p) & =\frac{i}{(2 \pi)^{7} \eta\left(\tau_{\varepsilon}\right)^{12}} \frac{\partial}{\partial \tau_{\varepsilon}}\left[e^{i \pi \tau_{\varepsilon} p^{2}} \sum_{i=2,3,4} \sum_{\alpha= \pm} \alpha \eta_{L}\left[\delta_{i}^{\alpha} ; \lambda\right]\left\langle\nu_{0} \mid \mu_{i}\right\rangle \vartheta_{i}^{4}\right] \\
d \mu_{L}^{(2)}[\lambda](p) & =\frac{e^{i \pi \tau_{\varepsilon} p^{2}}}{16 \pi^{6} \Psi_{10}} \frac{\vartheta\left[\delta_{j}^{+}\right]^{2} \vartheta\left[\delta_{j}^{-}\right]^{2}}{\vartheta_{j}^{4}} \sum_{\delta} \eta_{L}[\delta ; \lambda] \Xi_{6}[\delta] \vartheta[\delta]^{2} \vartheta[\delta+\varepsilon]^{2}
\end{aligned}
$$

It remains to put constraints on the GSO phases and determine all possible solutions to these constraints.

\subsection{Modular Covariance constraints on GSO phases}

Constraints on the GSO phases arise from the requirement of modular invariance of the full string measure. Under a general modular transformation $M \in S p(4, \mathbf{Z})$, the period matrix $\Omega$, the internal momenta $p_{L}$, the spin structure $\delta$, the sector label $\lambda=\left(a_{I}, b_{I}\right)$ and the twist $\varepsilon$ are transformed to $\tilde{\Omega}, \tilde{p}_{L}, \tilde{\delta}, \tilde{\lambda}$ and $\tilde{\varepsilon}$, according to familiar rules, which are summarized in Appendix B. Modular invariance requires the following transformation law for the GSO resummed measure,

$$
d \mu_{L}[\tilde{\lambda}]\left(\tilde{p}_{L}, \tilde{\Omega}\right)=\varphi(\lambda, M)\left(c \tau_{\varepsilon}+d\right)^{-2} d \mu_{L}[\lambda]\left(p_{L}, \Omega\right)
$$

The factor $\left(c \tau_{\varepsilon}+d\right)^{-2}$ represents the effect of the modular transformation induced by $M$ on the Prym period $\tau_{\varepsilon}$, and $\varphi$ is a set of phases.

The constraints on the GSO phases $\eta$ are most easily established by restricting $M$ to the subgroup $H_{\varepsilon}$ of the modular group which leaves $\varepsilon$ invariant. The two terms $d \mu_{L}^{(1)}$ and $d \mu_{L}^{(2)}$ are functionally independent, and therefore must each result from a modular

covariant GSO summation over $\delta$. The modular transformations of $d \mu_{L}^{(2)}$ are obtained by combining the relation

$$
\Xi_{6}[\tilde{\delta}](\tilde{\Omega}) \vartheta[\tilde{\delta}](0, \tilde{\Omega})^{4}=\operatorname{det}(C \Omega+D)^{8} \Xi_{6}[\delta](\Omega) \vartheta[\delta](0, \Omega)^{4}
$$

familiar from [4] with the transformation law of the $\vartheta$-constants,

$$
\frac{\vartheta[\tilde{\delta}+\varepsilon](0, \tilde{\Omega})}{\vartheta[\tilde{\delta}](0, \tilde{\Omega})}=\frac{\epsilon(\delta+\varepsilon, M)}{\epsilon(\delta, M)} \frac{\vartheta[\delta+\varepsilon](0, \Omega)}{\vartheta[\delta](0, \Omega)}
$$

Therefore, modular covariance requires that

$$
\eta_{L}[\tilde{\delta} ; \tilde{\lambda}] \frac{\epsilon(\delta+\varepsilon, M)^{2}}{\epsilon(\delta, M)^{2}}=\varphi(\lambda, M) \eta_{L}[\delta ; \lambda] \quad \text { for all } \quad M \in H_{\varepsilon}
$$

The ratios of $\epsilon^{2}$ may be computed by inspecting Table 3 . The symmetric pairs $(\delta, \delta+\varepsilon)$ take the values $\left(\delta_{1}, \delta_{2}\right),\left(\delta_{3}, \delta_{4}\right)$ and $\left(\delta_{7}, \delta_{8}\right)$. It is manifest from Table 3 that the $\epsilon^{2}$ values 
for both members of each pair coincide for all $M \in H_{\varepsilon}$. Thus, we have the following condition for each given $\lambda$,

$$
\eta_{L}[\tilde{\delta} ; \tilde{\lambda}]=\varphi(\lambda, M) \eta_{L}[\delta ; \lambda] \quad \text { for all } \quad M \in H_{\varepsilon}
$$

It is straightforward to show that the second part of the chiral measure $d \mu_{L}^{(1)}$ is then automatically modular covariant. To solve these constraints requires more detailed information on the structure of the orbifold group $G$. Two specific examples will be discussed in the remainder of this section.

From Table 3, it is clear that the modular subgroup, as it acts on the even spin structures, has two distinct orbits. Actually, these orbits are distinguished by the signatures $\langle\varepsilon \mid \delta\rangle$ in the following manner,

$$
\begin{array}{lll}
\langle\varepsilon \mid \delta\rangle=+1 & \text { orbit } & \left\{\delta_{1}, \delta_{2}, \delta_{3}, \delta_{4}, \delta_{7}, \delta_{8}\right\} \\
\langle\varepsilon \mid \delta\rangle=-1 & \text { orbit } & \left\{\delta_{5}, \delta_{6}, \delta_{9}, \delta_{0}\right\}
\end{array}
$$

The orbit $\left\{\delta_{1}, \delta_{2}, \delta_{3}, \delta_{4}, \delta_{7}, \delta_{8}\right\}$ is precisely the one that contains all even spin structures $\delta$ for which $\delta+\varepsilon$ is also even; these are the only spin structures that enter here.

\section{5 $\mathrm{Z}_{2}$ Orbifolds}

We now consider the model where 4 directions are compactified by an orbifold group $G$ whose point $P_{G}$ group consists of single $\mathbf{Z}_{\mathbf{2}}$ chiral reflection $r_{L}$ of the fields $x$ and $\psi$. Since $r_{L}^{2}=1$, all sectors may be labeled precisely by a single twist $\varepsilon$, i.e. $\lambda=\varepsilon$. As a result, the modular covariance condition (8.19) further simplifies and becomes,

$$
\eta_{L}[\tilde{\delta} ; \varepsilon]=\varphi(\varepsilon, M) \eta_{L}[\delta ; \varepsilon] \quad \text { for all } \quad M \in H_{\varepsilon}
$$

By a modular transformation, $\varepsilon$ may be brought to the standard form (6.1). The spin structures transform under $H_{\varepsilon}$ in the two orbits listed in (8.20), and (8.21) is to be solved separately in each orbit.

Observe that if a modular transformation $M \in H_{\varepsilon}$ leaves any one of the spin structures $\delta$ invariant, one has $\eta_{L}[\delta ; \varepsilon]=\varphi(\varepsilon, M) \eta_{L}[\delta ; \varepsilon]$, and thus $\varphi(\varepsilon, M)=1$. (The alternative, $\eta_{L}[\delta ; \varepsilon]=0$ would lead to zero GSO phases throughout the orbit, and thus a vanishing partition function, which is excluded.) It also follows that any spin structures related by $M$ will have the same GSO phase $\eta_{L}[\delta ; \varepsilon]=\eta_{L}[\tilde{\delta} ; \varepsilon]$.

Applying these observations to the case of orbit $\left\{\delta_{1}, \delta_{2}, \delta_{3}, \delta_{4}, \delta_{7}, \delta_{8}\right\}$, simple inspection of Table 3 reveals that $M_{1}, M_{3}$ and $T_{2}$ have fixed points, so that $\varphi\left(\varepsilon, M_{1}\right)=\varphi\left(\varepsilon, M_{3}\right)=$ $\varphi\left(\varepsilon, T_{2}\right)=1$, and $\eta_{L}\left[\delta_{1} ; \varepsilon\right]=\eta_{L}\left[\delta_{2} ; \varepsilon\right], \eta_{L}\left[\delta_{3} ; \varepsilon\right]=\eta_{L}\left[\delta_{4} ; \varepsilon\right]$, and $\eta_{L}\left[\delta_{7} ; \varepsilon\right]=\eta_{L}\left[\delta_{8} ; \varepsilon\right]$. From the first two relations, it follows that $\varphi\left(\varepsilon, S_{2}\right)=1$, while from the last that $\varphi\left(\varepsilon, M_{2}\right)=1$. 
Using the action of $S_{2}$ and $M_{2}$ it follows that $\eta_{L}[\delta ; \varepsilon]$ is independent of $\delta$ within this entire orbit, and may be set equal to $\eta_{L}[\delta ; \varepsilon]=1$. (The analysis in the case of the orbit $\left\{\delta_{5}, \delta_{6}, \delta_{9}, \delta_{0}\right\}$ leads to the conclusion that $\eta_{L}[\delta ; \varepsilon]$ is constant throughout also this orbit.) Since the chiral measure for $\mathbf{Z}_{2}$ reflections is non-zero for only the first orbit above, the GSO resummed measure becomes simply,

$$
d \mu_{L}[\varepsilon]\left(p_{L}\right)=\sum_{\delta} d \mu_{L}[\delta, \varepsilon]\left(p_{L}\right)
$$

Notice that with a single twist, the assignment of GSO phases consistent with modular invariance (in the even spin structure sector) is unique, a situation that is familiar from flat space-time [4].

For the above GSO phases, the chirally summed measure $d \mu_{C}^{(2)}$ vanishes pointwise on moduli space. To prove this, the starting point is a relation, derived first in [4],

$$
\sum_{\delta} \Xi_{6}[\delta] \vartheta[\delta]^{4} S_{\delta}(z, w)^{2}=\sum_{\delta} \Xi_{6}[\delta] \vartheta[\delta]^{2}(0) \vartheta[\delta]^{2}\left(\int_{w}^{z} \omega_{I}\right)=0
$$

for any pair of points $z, w$ on the surface. It suffices to choose the pair to be branch points, canonically associated with the twist via $\varepsilon=-\nu_{a}+\nu_{b}$ and $z=\Delta+\nu_{b}$ and $w=\Delta+\nu_{a}$, where $\Delta$ is the Riemann class. Using (B.9) and the specific form of the standard twist $\varepsilon$, the desired relation is readily obtained,

$$
\sum_{\delta} \Xi_{6}[\delta] \vartheta[\delta]^{2} \vartheta[\delta+\varepsilon]^{2}=0
$$

which proves that $d \mu_{L}^{(2)}[\varepsilon]\left(p_{L}\right)=0$. The vanishing of the measure $d \mu_{L}^{(1)}[\varepsilon]\left(p_{L}\right)$ is even simpler, as it results directly from the Riemann identities for the Prym variety,

$$
\sum_{i=2,3,4}\left\langle\nu_{0} \mid \mu_{i}\right\rangle \vartheta_{i}\left(0, \tau_{\varepsilon}\right)^{4}=0
$$

The vanishing of $d \mu_{L}^{(1,2)}$ might have been expected, on the grounds that the model obtained by orbifolding by a single $\mathbf{Z}_{2}$ reflection group produces an orbifold superstring theory that coincides with the original Type II theory. ${ }^{12}$

\section{6 $\quad \mathrm{Z}_{2} \times \mathrm{Z}_{2}$ Orbifolds : KKS models}

The models introduced by Kachru-Kumar-Silverstein (KKS) in [9] are constructed on a square torus $T_{G}$ at self-dual radius and an asymmetric orbifold group $G$ whose point group

\footnotetext{
${ }^{12}$ We are grateful to Eva Silverstein and Shamit Kachru for detailed explanations of this point.
} 
$\bar{P}_{G}$ is generated by two elements,

$$
\begin{aligned}
& f=\left(\left(r_{L}, s_{R}\right)^{1-4},\left(1, s_{R}^{2}\right)^{5},\left(s_{L}, s_{R}\right)^{6} ;(-)^{F_{R}}\right) \\
& g=\left(\left(s_{L}, r_{R}\right)^{1-4},\left(s_{L}, s_{R}\right)^{5},\left(s_{L}^{2}, 1\right)^{6} ;(-)^{F_{L}}\right)
\end{aligned}
$$

The superscripts indicate the dimension labels on which the corresponding generator acts. The operators $(-)^{F_{L}}$ and $(-)^{F_{R}}$ denote the parity of left and right chirality worldsheet fermion number respectively, with value + on NS states and - on R states. The operations $r_{L}, r_{R}, s_{L}$ and $s_{R}$ are chiral reflections and shifts, defined below.

\subsubsection{Chiral Reflections and Shifts}

The chiral reflections $r_{L}$ and $r_{R}$ act on $x^{\mu}$ and $\psi^{\mu}$ by reflecting respectively the left and right oscillators of both fields and the momenta of $x^{\mu}$. Since $r_{L}$ and $r_{R}$ are reflections, their order is 2 . The product $r=r_{L} r_{R}$ is the usual reflection on the non-chiral fields, given by $r x^{\mu}=-x^{\mu}$ and $r \psi^{\mu}=-\psi^{\mu}$. The operators $s_{L}$ and $s_{R}$ leave the full fields $\psi^{\mu}$, as well as the oscillators of $x^{\mu}$ invariant. Their action on $x^{\mu}$ is solely through chiral shifts of the zero mode. The operators may be represented in terms of the left and right momentum operators $p_{L}^{\mu}$ and $p_{R}^{\mu}$,

$$
s_{L}=\exp \left\{2 \pi i \sum_{\mu} p_{L}^{\mu} R / 2\right\} \quad s_{R}=\exp \left\{2 \pi i \sum_{\mu} p_{R}^{\mu} R / 2\right\}
$$

The product $s=s_{L} s_{R}$ is the usual shift by half a circumference. At the self-dual radius $R=1 / \sqrt{2}$, both operators $s_{L}$ and $s_{R}$ are of order 4 . Compactification to a radius $R$ restricts the momentum parameters $\left(p_{L}, p_{R}\right)$ to a discrete Lorentzian lattice $\Gamma_{R}$. To simplify the notation, we shall not indicate this explicitly.

\subsubsection{GSO phases}

Of relevance here is the action of each generator on the left chiral blocks, including their spin structure dependence. The action of the chiral shift operator $s_{L}$ is diagonal on the chiral blocks and will enter only when assembling left and right chiral blocks together. Thus, when carrying out the GSO projection by summation over spin structures in each chirality sector separately, the action of the chiral shift operators is immaterial. Effectively, the action of the orbifold group $G$ on left chiral blocks reduces to the action of the point group $P_{G}$, whose generators are

$$
\begin{array}{lll}
f=\left(f_{L} ; f_{R}\right) & f_{L}=r_{L}^{1-4} & f_{R}=(-)^{F_{R}} \\
g=\left(g_{L} ; g_{R}\right) & g_{L}=(-)^{F_{L}} & g_{R}=r_{R}^{1-4}
\end{array}
$$

Clearly, each generator is of order 2, whence the fact that on the left chiral blocks, the action of the orbifold group is by the Abelian point group $P_{G}=\mathbf{Z}_{2} \times \mathbf{Z}_{2}$. 
All possible sectors of the $\mathbf{Z}_{2} \times \mathbf{Z}_{2}$ theory on the genus 2 surface may be parametrized by two half-characteristics $\varepsilon$ and $\alpha$. To establish this, denote the assignments of elements of $\mathbf{Z}_{2} \times \mathbf{Z}_{2}$ around each homology cycle as before, $\left(A_{I}, B_{I}\right) \rightarrow\left(a_{I}, b_{I}\right)$ for $I=1,2$ and $a_{I}, b_{I} \in \mathbf{Z}_{2} \times \mathbf{Z}_{2}$. One has the following correspondence between the twist values $\varepsilon, \alpha$ and the group element assignments on the homology cycles,

$$
\begin{array}{ll}
a_{1}=\left(f_{L}^{2 \varepsilon_{1}^{\prime}} g_{L}^{2 \alpha_{1}^{\prime}} ; f_{R}^{2 \varepsilon_{1}^{\prime}} g_{R}^{2 \alpha_{1}^{\prime}}\right) & b_{1}=\left(f_{L}^{2 \varepsilon_{1}^{\prime \prime}} g_{L}^{2 \alpha_{1}^{\prime \prime}} ; f_{R}^{2 \varepsilon_{1}^{\prime \prime}} g_{R}^{2 \alpha_{1}^{\prime \prime}}\right) \\
a_{2}=\left(f_{L}^{2 \varepsilon_{2}^{\prime}} g_{L}^{2 \alpha_{2}^{\prime}} ; f_{R}^{2 \varepsilon_{2}^{\prime}} g_{R}^{2 \alpha_{2}^{\prime}}\right) & b_{2}=\left(f_{L}^{2 \varepsilon_{2}^{\prime \prime}} g_{L}^{2 \alpha_{2}^{\prime \prime}} ; f_{R}^{2 \varepsilon_{2}^{\prime \prime}} g_{R}^{2 \alpha_{2}^{\prime \prime}}\right)
\end{array}
$$

Given a spin structure $\delta$, the effect of $g_{L}$ is to assign a sign factor depending on whether the state traversing the corresponding cycle is NS or R. This assignment takes a simple form in terms of the signature,

$$
\begin{aligned}
\text { NS state traversing cycle } \alpha & \langle\alpha \mid \delta\rangle=+1 \\
\mathrm{R} \text { state traversing cycle } \alpha & \langle\alpha \mid \delta\rangle=-1
\end{aligned}
$$

To check this, it suffices to work out a specific twist assignment, e.g. the standard twist $\alpha=\varepsilon$ of (6.1), whose only non-zero entry is $\varepsilon_{2}^{\prime \prime}=\frac{1}{2}$. If $\delta_{2}^{\prime}=0$, the state traversing the $A_{2}$ cycle is indeed NS and the signature assignment comes out to be +1 , while if $\delta_{2}^{\prime}=\frac{1}{2}$, the state is $\mathrm{R}$ and we get -1 .

The net effect in the sector $\left(a_{I}, b_{I}\right)$, parametrized by two twists $\varepsilon$ and $\alpha$, is to produce a $\mathbf{Z}_{2}$ reflection of the fields $x$ and $\psi_{+}$on the cycle $\varepsilon$, and an insertion of the phase $\langle\alpha \mid \delta\rangle$. As $\left(a_{I}, b_{I}\right)$ will run over all sectors, $\lambda \equiv(\varepsilon, \alpha)$ will run over all pairs of half-characteristics. It follows that the set of chiral blocks for the $\mathbf{Z}_{2} \times \mathbf{Z}_{2}^{\prime}$ theory is given by

$$
d \mu_{L}[\delta ; \varepsilon, \alpha]\left(p_{L}\right)=\langle\alpha \mid \delta\rangle d \mu_{L}[\delta ; \varepsilon]\left(p_{L}\right)
$$

Assembling all ingredients, the GSO summed chiral measure for given twists $\varepsilon$ and $\alpha$ is obtained as follows,

$$
d \mu_{L}[\varepsilon, \alpha]\left(p_{L}\right)=\sum_{\delta} d \mu_{L}[\delta ; \varepsilon, \alpha]\left(p_{L}\right)=\sum_{\delta}\langle\alpha \mid \delta\rangle d \mu_{L}[\delta, \varepsilon]\left(p_{L}\right)
$$

Under modular transformations that leave the twist $\varepsilon$ invariant, the measure transforms covariantly,

$$
d \mu_{L}[\varepsilon, \tilde{\alpha}]\left(\tilde{p}_{L}, \tilde{\Omega}\right) \equiv \varphi(\varepsilon, \alpha, M)\left(c \tau_{\varepsilon}+d\right)^{-2} d \mu_{L}[\varepsilon, \alpha]\left(p_{L}, \Omega\right)
$$

Here, the phase factor $\varphi(\varepsilon, \alpha, M)=\left\langle\alpha \mid(M)_{0}\right\rangle$ depends only on $M$ and $\alpha .{ }^{13}$ The presence of the phase factor $\langle\alpha \mid \delta\rangle$ represents a generalization of discrete torsion [43] to the chiral case.

\footnotetext{
${ }^{13}$ To be precise, $(M)_{0}$ is the inhomogeneous contribution in the modular transformation of spin structures given in (B.12), which is common to all spin structures.
} 
By analyzing the action of the modular subgroup that leaves two twists invariant, as done in $\S 6.4$, one may show that the above phase assignment is the unique non-trivial choice consistent with modular invariance. The proof is similar to the one given to determine the unique phase assignment for the $\mathbf{Z}_{\mathbf{2}}$ case in $\S 8.5$. This will be proven here for the case $\alpha \in \mathcal{O}_{+}[\varepsilon]$ only; the other case is analogous. Upon making the choice $\alpha=\varepsilon_{3}$, the stabilizer of both twists, $H_{\varepsilon, \alpha}$, is generated by $M_{1}, M_{2}$ and $M_{3}$. Acting on the spin structures $\left\{\delta_{1}, \delta_{2}, \delta_{3}, \delta_{4}, \delta_{7}, \delta_{8}\right\}, M_{1}$ and $M_{3}$ have fixed points. Therefore, $\varphi\left(\varepsilon, \alpha ; M_{1}\right)=\varphi\left(\varepsilon, \alpha ; M_{3}\right)=$ 1 and thus $\eta_{L}\left[\delta_{1} ; \varepsilon, \alpha\right]=\eta_{L}\left[\delta_{3} ; \varepsilon, \alpha\right], \eta_{L}\left[\delta_{2} ; \varepsilon, \alpha\right]=\eta_{L}\left[\delta_{4} ; \varepsilon, \alpha\right]$, and $\eta_{L}\left[\delta_{7} ; \varepsilon, \alpha\right]=\eta_{L}\left[\delta_{8} ; \varepsilon, \alpha\right]$. In view of the last equality, we must have $\varphi\left(\varepsilon, \alpha ; M_{2}\right)=1$, and we therefore conclude that

$$
\eta_{L}\left[\delta_{1} ; \varepsilon, \alpha\right]=\eta_{L}\left[\delta_{2} ; \varepsilon, \alpha\right]=\eta_{L}\left[\delta_{3} ; \varepsilon, \alpha\right]=\eta_{L}\left[\delta_{4} ; \varepsilon, \alpha\right] \quad \eta_{L}\left[\delta_{7} ; \varepsilon, \alpha\right]=\eta_{L}\left[\delta_{8} ; \varepsilon, \alpha\right]
$$

If all $\eta$ are equal, we recover the case of twisting by a single $\mathbf{Z}_{2}$. The only other linearly independent case is when

$$
\eta_{L}\left[\delta_{i} ; \varepsilon, \alpha\right]=+1, i=1,2,3,4 \quad \eta_{L}\left[\delta_{7} ; \varepsilon, \alpha\right]=\eta_{L}\left[\delta_{8} ; \varepsilon, \alpha\right]=-1
$$

but this assignment is readily seen to coincide with $\eta_{L}[\delta ; \varepsilon, \alpha]=\langle\alpha \mid \delta\rangle$, which is what we set out to prove.

\subsection{Analysis of the GSO resummed measure for KKS models}

For each sector $\lambda=(\varepsilon, \alpha)$, the chiral block $d \mu_{C}[\varepsilon, \alpha]\left(p_{L}, \Omega\right)$ may be analyzed by exploiting its modular properties. Using a first modular transformation, $\varepsilon$ may be mapped into the standard form, familiar from (6.1),

$$
\varepsilon=\left(\begin{array}{l|l}
0 & 0 \\
0 & \frac{1}{2}
\end{array}\right)
$$

Under the modular subgroup $H_{\varepsilon}$ (which leaves $\varepsilon$ invariant), twists transform in 4 orbits, as was shown in $\S 6.3$. The contribution to the GSO resummed measure must be analyzed orbit by orbit.

(1) $\alpha \in \mathcal{O}_{0}[\varepsilon]$.

This orbit corresponds to the untwisted sector and has only one element, $\alpha=0$. Using the results of section 7.2 , one establishes $d \mu_{L}[\varepsilon, 0]\left(p_{L}, \Omega\right)=0$.

(2) $\alpha \in \mathcal{O}_{\varepsilon}[\varepsilon]$.

This orbit also has only one element, $\alpha=\varepsilon$. Using the results (8.20), and (7.2), one establishes $d \mu_{L}[\varepsilon, \varepsilon]\left(p_{L}, \Omega\right)=0$. 
(3) $\alpha \in \mathcal{O}_{-}[\varepsilon]$.

The twists in this orbit are such that $\langle\alpha \mid \varepsilon\rangle=-1$. One may choose the representative

$$
\alpha=\left(\begin{array}{c|c}
0 & 0 \\
\frac{1}{2} & 0
\end{array}\right)
$$

The signature with the spin structures becomes explicit : $\left\langle\delta_{i}^{ \pm} \mid \alpha\right\rangle= \pm 1$. Therefore, the summation over $i=2,3,4$ vanishes and we have $d \mu_{L}^{(1)}[\varepsilon]\left(p_{L}, \Omega\right)=0$. The measure $d \mu_{L}^{(2)}[\varepsilon]\left(p_{L}, \Omega\right)$ involves the sum

$$
\sum_{i=2,3,4}\left(\Xi_{6}\left[\delta_{i}^{+}\right]-\Xi_{6}\left[\delta_{i}^{-}\right]\right) \vartheta\left[\delta_{i}^{+}\right]^{2} \vartheta\left[\delta_{i}^{-}\right]^{2}
$$

In the separating degeneration, this sum vanishes to order $\tau^{2}$ included. This may be seen from the following limit, derived from (7.2) for each $j=3,4$,

$$
\sum_{i=2,3,4} \Xi_{6}\left[\begin{array}{l}
\mu_{i} \\
\mu_{j}
\end{array}\right] \vartheta\left[\begin{array}{l}
\mu_{i} \\
00
\end{array}\right]^{2} \vartheta\left[\begin{array}{l}
\mu_{i} \\
0 \frac{1}{2}
\end{array}\right]^{2}=\mathcal{O}\left(\tau^{4}\right)
$$

using the Riemann identities of (7.9) for the summation over $i=2,3,4$. It may be shown that the order $\tau^{4}$ term, on the other hand is non-vanishing.

(4) $\alpha \in \mathcal{O}_{+}[\varepsilon]$.

The twists in this orbit are such that $\langle\alpha \mid \varepsilon\rangle=+1$. One may choose the representative

$$
\alpha=\left(\begin{array}{l|l}
0 & \frac{1}{2} \\
0 & 0
\end{array}\right)
$$

so that $\langle\delta \mid \alpha\rangle=(-)^{2 \delta_{1}^{\prime}}$. The measure $d \mu_{L}^{(1)}[\varepsilon]\left(p_{L}, \Omega\right)$ manifestly vanishes, as the contributions from $\alpha=+$ and $\alpha=-$ cancel one another out. The measure $d \mu_{L}^{(2)}[\varepsilon]\left(p_{L}, \Omega\right)$ on the other hand vanishes only to leading order as $\tau \rightarrow 0$, but is non-vanishing to order $\tau^{2}$. To see this, the following limit is taken

$$
\begin{aligned}
& \sum_{\delta}\langle\alpha \mid \delta\rangle \Xi_{6}[\delta] \vartheta[\delta]^{2} \vartheta[\delta+\varepsilon]^{2} \\
& \quad=-256 \pi^{2} \tau^{2} \eta\left(\tau_{1}\right)^{12} \eta\left(\tau_{2}\right)^{12}\left(\vartheta_{3}^{8}-\vartheta_{4}^{8}\right)\left(\tau_{1}\right) \vartheta_{2}^{4} \vartheta_{3}^{2} \vartheta_{4}^{2}\left(\tau_{2}\right)+\mathcal{O}\left(\tau^{4}\right)
\end{aligned}
$$

Thus, the chiral measure $d \mu_{L}[\varepsilon, \alpha]\left(p_{\varepsilon}\right)$ is non-vanishing on both orbits $\alpha \in \mathcal{O}_{ \pm}[\varepsilon]$.

\subsection{Conclusions on the KKS model}

The non-vanishing of the contribution from the orbit $\mathcal{O}_{-}[\varepsilon]$ would appear to contradict the conclusions of [9], where, instead, it is claimed to be vanishing. The arguments made 
in [9] are based on the fact that, when viewed as elements of the full orbifold group $G$, the generators $f$ and $g$ do not commute with one another. Since their commutator is a pure translation, the elements $f$ and $g$ do commute, however, when viewed as elements of the point group $\bar{P}_{G}$.

Standard arguments for symmetric orbifolds, based on functional integral methods, guarantee the equivalence between coseting $\mathbf{R}^{n}$ by $\mathrm{G}$ and coseting $T_{G}$ by $\bar{P}_{G}$. They also guarantee that no contributions will arise from any sector in which the homotopy relation $\prod_{I=1,2} a_{I} b_{I} a_{I}^{-1} b_{I}^{-1}=1$ fails to be satisfied, whether $a_{I}, b_{I}$ take values in $G$ or in $\bar{P}_{G}$.

For asymmetric orbifolds, however, no direct functional integral formulation is available. Therefore, the arguments made in the case of symmetric orbifolds may or may not hold for asymmetric orbifolds. In particular, it is unclear how the coset theory $\mathbf{R}^{n} / G$ should be constructed, since it is unclear how the zero mode of the field $x$ should be handled. Only the construction of the coset $T_{G} / \bar{P}_{G}$ is well-defined. Its construction guarantees that no contributions will arise from a sector in which the homotopy relation $\prod_{I=1,2} a_{I} b_{I} a_{I}^{-1} b_{I}^{-1}=1$ fails to be satisfied, only when $a_{I}, b_{I}$ take values in the space group $P_{G}$ (but not necessarily when $f$ and $g$ take values in the full group $G$ ).

To summarize the situation for orbit $\mathcal{O}_{-}[\varepsilon]$ : the GSO resummed chiral blocks in orbit $\mathcal{O}_{-}[\varepsilon]$ are non-vanishing. Yet, it is conceivable that assembling left and right blocks of the asymmetric orbifold will effectively enforce the vanishing of contributions arising from sectors in which the homotopy relation $\prod_{I=1,2} a_{I} b_{I} a_{I}^{-1} b_{I}^{-1}=1$ fails to be satisfied, when $a_{I}, b_{I}$ take values in the full group $G$. No evidence in favor of this possibility is available, however, at this time.

In the orbit $\mathcal{O}_{+}[\varepsilon]$, the chiral GSO resummed blocks are also non-vanishing. As their contribution is $\mathcal{O}\left(\tau^{2}\right)$ in the separating degeneration limit, they dominate in this limit (over the orbit $\mathcal{O}_{-}[\varepsilon]$, which is $\mathcal{O}\left(\tau^{4}\right)$ ). This result contradicts the claim of [9], where the corresponding chiral contribution was found to vanish. Noticing that the sectors for this block all satisfy the homotopy relation, no cancellation should be expected when assembling left and right blocks.

To summarize, the non-vanishing of the contribution of orbit $\mathcal{O}_{+}[\varepsilon]$ shows that the cosmological constant cannot vanish pointwise on moduli space and gives strong evidence that the full cosmological constant in the KKS models is non-vanishing. The net effect of the correct treatment of the superstring measure given here is that the cosmological constant in KKS models involves the sum over the new modular object $\Xi_{6}[\delta]$, discovered in [4], which, in contrast to the measure derived from the BRST picture changing operator formalism does not lead to a cancellation in the chiral blocks.

\section{Acknowledgments}

We are happy to acknowledge conversations with Michael Gutperle, Per Kraus, and Edward Witten. We are especially grateful to Shamit Kachru and Eva Silverstein for 
several helpful discussions of various fine points in the theory of asymmetric orbifold compactifications and for detailed explanations of their specific models.

\section{A Appendix : Genus $1 \vartheta$-function identities}

Let $\omega$ and $\omega^{\prime}$ be the two half period of the torus and $\tau=\omega^{\prime} / \omega$ its modulus. The Weierstrass function obeys

$$
\wp^{\prime}(z)^{2}=4\left(\wp(z)-e_{1}\right)\left(\wp(z)-e_{2}\right)\left(\wp(z)-e_{3}\right)
$$

Recall the prime form $E$ and the Szego kernel $S_{\mu}$ for even spin structure $\mu$ at genus 1,

$$
E(z, w)=\frac{\vartheta_{1}(z-w \mid \tau)}{\vartheta_{1}^{\prime}(0 \mid \tau)} \quad S_{\mu}(z, w)=\frac{\vartheta[\mu](z-w \mid \tau)}{\vartheta[\mu](0 \mid \tau) E(z, w)}
$$

The correspondence between the classical Jacobi $\vartheta_{i}$-functions and the spin structures is given by $\vartheta_{i}(z \mid \tau) \equiv \vartheta\left[\mu_{i}\right](z \mid \tau)$ with

$$
\mu_{1}=\left(\frac{1}{2} \mid \frac{1}{2}\right) \quad \mu_{2}=\left(\frac{1}{2} \mid 0\right) \quad \mu_{3}=(0 \mid 0) \quad \mu_{4}=\left[0 \frac{1}{2}\right]
$$

The Dedekind $\eta$-function is related to the product of all $\vartheta$-constants,

$$
\vartheta_{1}^{\prime}(0 \mid \tau)=-2 \pi \eta(\tau)^{3}=-\pi \vartheta_{2}(0 \mid \tau) \vartheta_{3}(0 \mid \tau) \vartheta_{4}(0 \mid \tau)
$$

We have the following doubling formulas,

$$
\begin{aligned}
& \vartheta_{1}(2 z \mid 2 \tau)=\vartheta_{1}(z \mid \tau) \vartheta_{2}(z \mid \tau) / \vartheta_{4}(0 \mid 2 \tau) \\
& \vartheta_{2}(2 z \mid 2 \tau)=\left(\vartheta_{3}(z \mid \tau)^{2}-\vartheta_{4}(z \mid \tau)^{2}\right) / \vartheta_{2}(0 \mid 2 \tau) \\
& \vartheta_{3}(2 z \mid 2 \tau)=\left(\vartheta_{3}(z \mid \tau)^{2}+\vartheta_{4}(z \mid \tau)^{2}\right) / \vartheta_{3}(0 \mid 2 \tau) \\
& \vartheta_{4}(2 z \mid 2 \tau)=\vartheta_{3}(z \mid \tau) \vartheta_{4}(z \mid \tau) / \vartheta_{4}(0 \mid 2 \tau)
\end{aligned}
$$

The doubling formulas for the $\vartheta$-constants are as follows,

$$
\begin{aligned}
2 \vartheta_{2}(0 \mid 2 \tau)^{2} & =\vartheta_{3}(0 \mid \tau)^{2}-\vartheta_{4}(0 \mid \tau)^{2} \\
2 \vartheta_{3}(0 \mid 2 \tau)^{2} & =\vartheta_{3}(0 \mid \tau)^{2}+\vartheta_{4}(0 \mid \tau)^{2} \\
\vartheta_{4}(0 \mid 2 \tau)^{2} & =\vartheta_{3}(0 \mid \tau) \vartheta_{4}(0 \mid \tau)
\end{aligned}
$$

We have the following derivation formula,

$$
\frac{\partial}{\partial z} \ln \frac{\vartheta_{1}(z \mid \tau)}{\vartheta[\mu](z \mid \tau)}=-\pi \vartheta[\mu]^{2}(0 \mid \tau) \frac{\vartheta\left[\mu^{\prime}\right](z \mid \tau) \vartheta\left[\mu^{\prime \prime}\right](z \mid \tau)}{\vartheta_{1}(z \mid \tau) \vartheta[\mu](z \mid \tau)}
$$

This formula is valid for any even spin structure $\mu$; the spin structures $\mu^{\prime}$ and $\mu^{\prime \prime}$ above are such that the triple $\left(\mu, \mu^{\prime}, \mu^{\prime \prime}\right)$ is a permutation of $\left(\mu_{2}, \mu_{3}, \mu_{4}\right)$. 


\section{B Appendix : Genus 2 Riemann Surfaces}

In this appendix, we review from [4] some fundamental facts about genus 2 Riemann surfaces, their spin structures, $\vartheta$-functions modular properties, and relations between the $\vartheta$-function and the hyperelliptic formulations.

\section{B.1 Spin Structures}

On a Riemann surface $\Sigma$ of genus 2, there are 16 spin structures, of which 6 are odd (usually denoted by $\nu$ ) and 10 are even (usually denoted by $\delta$ ). Each spin structure $\kappa$ can be identified with a $\vartheta$-characteristic $\kappa=\left(\kappa^{\prime} \mid \kappa^{\prime \prime}\right)$, where $\kappa^{\prime}, \kappa^{\prime \prime} \in\left\{0, \frac{1}{2}\right\}^{2}$, represented here by column matrices. The parity of the spin structure $\kappa$ is that of the integer $4 \kappa^{\prime} \cdot \kappa^{\prime \prime}$. The signature assignment between (even or odd) spin structures $\kappa$ and $\lambda$ is defined by

$$
\langle\kappa \mid \lambda\rangle \equiv \exp \left\{4 \pi i\left(\kappa^{\prime} \lambda^{\prime \prime}-\kappa^{\prime \prime} \lambda^{\prime}\right)\right\}
$$

- If $\kappa_{1}$ and $\kappa_{2}$ have the same parity (resp. opposite parity), we have

$$
\begin{aligned}
& \left\langle\kappa_{1} \mid \kappa_{2}\right\rangle=+1 \quad \Leftrightarrow \quad \kappa_{1}-\kappa_{2} \quad \text { even } \quad \text { (resp. odd) } \\
& \left\langle\kappa_{1} \mid \kappa_{2}\right\rangle=-1 \quad \Leftrightarrow \quad \kappa_{1}-\kappa_{2} \quad \text { odd } \quad \text { (resp. even) }
\end{aligned}
$$

- If $\nu_{1}, \nu_{2}$ and $\nu_{3}$ are odd and all distinct, then

$$
\left\langle\nu_{1} \mid \nu_{2}\right\rangle\left\langle\nu_{2} \mid \nu_{3}\right\rangle\left\langle\nu_{3} \mid \nu_{1}\right\rangle=-1
$$

It will be convenient to use a definite basis for the spin structures, in a given choice of homology basis, as in [4]. The odd spin structures may be labeled as follows,

$$
\begin{aligned}
2 \nu_{1}=\left(\begin{array}{l|l}
0 & 0 \\
1 & 1
\end{array}\right) & 2 \nu_{3}=\left(\begin{array}{l|l}
0 & 1 \\
1 & 1
\end{array}\right) & 2 \nu_{5}=\left(\begin{array}{l|l}
1 & 0 \\
1 & 1
\end{array}\right) \\
2 \nu_{2}=\left(\begin{array}{l|l}
1 & 1 \\
0 & 0
\end{array}\right) & 2 \nu_{4}=\left(\begin{array}{l|l}
1 & 1 \\
0 & 1
\end{array}\right) & 2 \nu_{6}=\left(\begin{array}{l|l}
1 & 1 \\
1 & 0
\end{array}\right)
\end{aligned}
$$

The pairs for which $\left\langle\nu_{i} \mid \nu_{j}\right\rangle=-1$ are 14, 16, 23, 25, 35, 46; all others give $\left\langle\nu_{i} \mid \nu_{j}\right\rangle=+1$. The even spin structures may be labeled by,

$$
\begin{array}{rlrl}
2 \delta_{1}=\left(\begin{array}{l|l}
0 & 0 \\
0 & 0
\end{array}\right) & 2 \delta_{2}=\left(\begin{array}{l|l}
0 & 0 \\
0 & 1
\end{array}\right) & 2 \delta_{3}=\left(\begin{array}{l|l}
0 & 1 \\
0 & 0
\end{array}\right) & 2 \delta_{4}=\left(\begin{array}{l|l}
0 & 1 \\
0 & 1
\end{array}\right) \\
2 \delta_{5}=\left(\begin{array}{l|l}
0 & 0 \\
1 & 0
\end{array}\right) & 2 \delta_{6}=\left(\begin{array}{l|l}
0 & 1 \\
1 & 0
\end{array}\right) & 2 \delta_{7}=\left(\begin{array}{l|l}
1 & 0 \\
0 & 0
\end{array}\right) & 2 \delta_{8}=\left(\begin{array}{l|l}
1 & 0 \\
0 & 1
\end{array}\right) \\
& 2 \delta_{9}=\left(\begin{array}{l|l}
1 & 0 \\
1 & 0
\end{array}\right) & 2 \delta_{0}=\left(\begin{array}{l|l}
1 & 1 \\
1 & 1
\end{array}\right)
\end{array}
$$

The pairs for which $\left\langle\delta_{i} \mid \delta_{j}\right\rangle=-1$ are $25,26,29,20,37,38,39,30,45,46,47,48,58,50$, $67,69,70,89$; all others give +1 . 
For genus 2 there exist many relations between even and odd spin structures, some of which will be needed here. First, the sum of all odd spin structures is a double period,

$$
\nu_{1}+\nu_{2}+\nu_{3}+\nu_{4}+\nu_{5}+\nu_{6}=4 \delta_{0}
$$

Second, each even spin structure $\delta$ can be written as $\delta=\nu_{i_{1}}+\nu_{i_{2}}+\nu_{i_{3}}$ (modulo integral periods), where the $\nu_{i_{a}}, a=1,2,3$ are odd and pairwise distinct,

$$
\begin{array}{ll}
\nu_{1}+\nu_{2}+\nu_{3}=\delta_{7}+2 \nu_{3} & \nu_{1}+\nu_{2}+\nu_{4}=\delta_{5}+2 \nu_{4} \\
\nu_{1}+\nu_{2}+\nu_{5}=\delta_{3}+2 \nu_{5} & \nu_{1}+\nu_{2}+\nu_{6}=\delta_{2}+2 \nu_{6} \\
\nu_{1}+\nu_{3}+\nu_{4}=\delta_{8}+2 \nu_{3} & \nu_{1}+\nu_{3}+\nu_{5}=\delta_{0}+2 \nu_{1} \\
\nu_{1}+\nu_{3}+\nu_{6}=\delta_{9}+2 \nu_{3} & \nu_{1}+\nu_{4}+\nu_{5}=\delta_{4}+2 \nu_{5} \\
\nu_{1}+\nu_{4}+\nu_{6}=\delta_{1}+2 \delta_{0} & \nu_{1}+\nu_{5}+\nu_{6}=\delta_{6}+2 \nu_{5}
\end{array}
$$

Clearly, the mapping $\left\{\nu_{i_{1}}, \nu_{i_{2}}, \nu_{i_{3}}\right\} \rightarrow \delta$ is 2 to 1 , with $\nu_{i_{1}}+\nu_{i_{2}}+\nu_{i_{3}}$ and its complement $4 \delta_{0}-\left(\nu_{i_{1}}+\nu_{i_{2}}+\nu_{i_{3}}\right)$ corresponding to the same even spin structure, in view of (B.6).

\section{B.2 $\vartheta$-functions}

The $\vartheta$-function is an entire function in the period matrix $\Omega$ and $\zeta \in \mathbf{C}^{2}$, defined by

$$
\vartheta[\kappa](\zeta, \Omega) \equiv \sum_{n \in \mathbf{Z}^{2}} \exp \left\{\pi i\left(n+\kappa^{\prime}\right) \Omega\left(n+\kappa^{\prime}\right)+2 \pi i\left(n+\kappa^{\prime}\right)\left(\zeta+\kappa^{\prime \prime}\right)\right\}
$$

Here, $\vartheta$ is even or odd in $\zeta$ depending on the parity of the spin structure. The following useful periodicity relations hold, in which $m^{\prime}, m^{\prime \prime} \in \mathbf{Z}^{2}$ and $\lambda^{\prime}, \lambda^{\prime \prime} \in \mathbf{C}^{2}$,

$$
\begin{aligned}
\vartheta[\kappa]\left(\zeta+m^{\prime \prime}+\Omega m^{\prime}, \Omega\right) & =\vartheta[\kappa](\zeta, \Omega) \exp \left\{-i \pi m^{\prime} \Omega m^{\prime}-2 \pi i m^{\prime}\left(\zeta+\kappa^{\prime \prime}\right)+2 \pi i \kappa^{\prime} m^{\prime \prime}\right\} \\
\vartheta\left[\kappa^{\prime}+m^{\prime}, \kappa^{\prime \prime}+m^{\prime \prime}\right](\zeta, \Omega) & =\vartheta\left[\kappa^{\prime}, \kappa^{\prime \prime}\right](\zeta, \Omega) \exp \left\{2 \pi i \kappa^{\prime} m^{\prime \prime}\right\} \\
\vartheta[\kappa+\lambda](\zeta, \Omega) & =\vartheta[\kappa]\left(\zeta+\lambda^{\prime \prime}+\Omega \lambda^{\prime}, \Omega\right) \exp \left\{i \pi \lambda^{\prime} \Omega \lambda^{\prime}+2 \pi i \lambda^{\prime}\left(\zeta+\lambda^{\prime \prime}+\kappa^{\prime \prime}\right)\right\}
\end{aligned}
$$

The $\vartheta$-function with vanishing characteristic is often denoted by $\vartheta(\zeta, \Omega)=\vartheta[0](\zeta, \Omega)$. For each odd spin structure $\nu$ we have $\vartheta[\nu](0, \Omega)=0$. For each even spin structure $\delta$ one defines the particularly important $\vartheta$-constants, $\vartheta[\delta] \equiv \vartheta[\delta](0, \Omega)$. For every odd spin structure, there exists a Riemann relation for $\vartheta$-constants,

$$
\sum_{\delta}\langle\nu \mid \delta\rangle \vartheta[\delta]^{4}=0
$$




\section{B.3 The Action of Modular Transformations}

Modular transformations $M$ form the infinite discrete group $S p(4, \mathbf{Z})$, defined by

$$
M=\left(\begin{array}{cc}
A & B \\
C & D
\end{array}\right) \quad\left(\begin{array}{cc}
A & B \\
C & D
\end{array}\right)\left(\begin{array}{cc}
0 & I \\
-I & 0
\end{array}\right)\left(\begin{array}{ll}
A & B \\
C & D
\end{array}\right)^{T}=\left(\begin{array}{cc}
0 & I \\
-I & 0
\end{array}\right)
$$

where $A, B, C, D$ are integer valued $2 \times 2$ matrices and the superscript ${ }^{t}$ denotes transposition. To exhibit the action of the modular group on $1 / 2$ characteristics, it is convenient to assemble the $1 / 2$ characteristics into a single column of 4 entries. In this notation, the action of the modular group on spin structures $\kappa$ and twists $\varepsilon$ is then given by [39]

$$
\begin{aligned}
\left(\begin{array}{c}
\tilde{\kappa}^{\prime} \\
\tilde{\kappa}^{\prime \prime}
\end{array}\right) & =\left(\begin{array}{cc}
D & -C \\
-B & A
\end{array}\right)\left(\begin{array}{c}
\kappa^{\prime} \\
\kappa^{\prime \prime}
\end{array}\right)+\frac{1}{2} \operatorname{diag}\left(\begin{array}{c}
C D^{T} \\
A B^{T}
\end{array}\right) \\
\left(\begin{array}{c}
\tilde{\varepsilon}^{\prime} \\
\tilde{\varepsilon}^{\prime \prime}
\end{array}\right) & =\left(\begin{array}{cc}
D & -C \\
-B & A
\end{array}\right)\left(\begin{array}{l}
\varepsilon^{\prime} \\
\varepsilon^{\prime \prime}
\end{array}\right)
\end{aligned}
$$

Here and below, $\operatorname{diag}(M)$ of a $n \times n$ matrix $M$ is an $1 \times n$ column vector whose entries are the diagonal entries on $M$. On the period matrix, modular transformations act by $\tilde{\Omega}=(A \Omega+B)(C \Omega+D)^{-1}$, while on the Jacobi $\vartheta$-functions, we have

$$
\vartheta[\tilde{\kappa}]\left(\left\{(C \Omega+D)^{-1}\right\}^{T} \zeta, \tilde{\Omega}\right)=\epsilon(\kappa, M) \operatorname{det}(C \Omega+D)^{\frac{1}{2}} e^{i \pi \zeta(C \Omega+D)^{-1} C \zeta} \vartheta[\kappa](\zeta, \Omega)
$$

where $\kappa=\left(\kappa^{\prime} \mid \kappa^{\prime \prime}\right)$ and $\tilde{\kappa}=\left(\tilde{\kappa}^{\prime} \mid \tilde{\kappa}^{\prime \prime}\right)$. The phase factor $\epsilon(\kappa, M)$ depends upon both $\kappa$ and the modular transformation $M$ and obeys $\epsilon(\kappa, M)^{8}=1$. Its expression was calculated in [39], and is given by $\epsilon(\delta, M)=\epsilon_{0}(M) \exp \left\{2 \pi i \phi_{\delta}(M)\right\}$, where

$$
\begin{aligned}
\epsilon_{0}(M)^{2} & =\exp \left\{2 \pi i \frac{1}{8} \operatorname{tr}(M-I)\right\} \\
\phi_{\delta}(M) & =-\frac{1}{2} \delta^{\prime} D^{T} B \delta^{\prime}+\delta^{\prime} B^{T} C \delta^{\prime \prime}-\frac{1}{2} \delta^{\prime \prime} C^{T} A \delta^{\prime \prime}+\frac{1}{2}\left(\delta^{\prime} D^{T}-\delta^{\prime \prime} C^{T}\right) \operatorname{diag}\left(A B^{T}\right)
\end{aligned}
$$

The modular group is generated by the following elements

$$
\begin{array}{rlrl}
M_{i} & =\left(\begin{array}{cc}
I & B_{i} \\
0 & I
\end{array}\right) & B_{1}=\left(\begin{array}{ll}
1 & 0 \\
0 & 0
\end{array}\right) & B_{2}=\left(\begin{array}{ll}
0 & 0 \\
0 & 1
\end{array}\right) \quad B_{3}=\left(\begin{array}{ll}
0 & 1 \\
1 & 0
\end{array}\right) \\
S & =\left(\begin{array}{cc}
0 & I \\
-I & 0
\end{array}\right) & & \\
\Sigma & =\left(\begin{array}{cc}
\sigma & 0 \\
0 & -\sigma
\end{array}\right) & \sigma=\left(\begin{array}{cc}
0 & 1 \\
-1 & 0
\end{array}\right) \\
T & =\left(\begin{array}{cc}
\tau_{+} & 0 \\
0 & \tau_{-}
\end{array}\right) & \tau_{+}=\left(\begin{array}{ll}
1 & 1 \\
0 & 1
\end{array}\right) \quad \tau_{-}=\left(\begin{array}{cc}
1 & 0 \\
-1 & 1
\end{array}\right)
\end{array}
$$

The transformation laws for even spin structures under these generators are given in Table 6; those for odd spin structures will not be needed here and may be found in [4]. 
We shall be most interested in the modular transformations of $\vartheta$-constants $\vartheta^{2}[\delta]$ and thus in even spin structures $\delta$ and the squares of $\epsilon$, which are given by

$$
\left\{\begin{array} { c } 
{ \epsilon ( \delta , M _ { 1 } ) ^ { 2 } = \operatorname { e x p } \{ 2 \pi i \delta _ { 1 } ^ { \prime } ( 1 - \delta _ { 1 } ^ { \prime } ) \} } \\
{ \epsilon ( \delta , M _ { 2 } ) ^ { 2 } = \operatorname { e x p } \{ 2 \pi i \delta _ { 2 } ^ { \prime } ( 1 - \delta _ { 2 } ^ { \prime } ) \} } \\
{ \epsilon ( \delta , M _ { 3 } ) ^ { 2 } = \operatorname { e x p } \{ - 4 \pi i \delta _ { 1 } ^ { \prime } \delta _ { 2 } ^ { \prime } \} }
\end{array} \quad \left\{\begin{array}{l}
\epsilon(\delta, S)^{2}=-1 \\
\epsilon(\delta, \Sigma)^{2}=-1 \\
\epsilon(\delta, T)^{2}=+1
\end{array}\right.\right.
$$

A convenient way of establishing these values is by first analyzing the case of the shifts $M_{i}$, whose action may be read off from the definition of the $\vartheta$-function. The values for the transformations $S, \Sigma$ and $T$ may be obtained by letting the surface undergo a separating degeneration $\Omega_{12} \rightarrow 0$, and using the sign assignments of genus $1 \vartheta$-functions. The nontrivial entries for $\epsilon^{2}$ are listed in Table 6 .

\begin{tabular}{|c||c|c|c|c|c|c||c|c|c|}
\hline$\delta$ & $M_{1}$ & $M_{2}$ & $M_{3}$ & $S$ & $\Sigma$ & $T$ & $\epsilon^{2}\left(\delta, M_{1}\right)$ & $\epsilon^{2}\left(\delta, M_{2}\right)$ & $\epsilon^{2}\left(\delta, M_{3}\right)$ \\
\hline \hline$\delta_{1}$ & $\delta_{3}$ & $\delta_{2}$ & $\delta_{1}$ & $\delta_{1}$ & $\delta_{1}$ & $\delta_{1}$ & 1 & 1 & 1 \\
\hline$\delta_{2}$ & $\delta_{4}$ & $\delta_{1}$ & $\delta_{2}$ & $\delta_{5}$ & $\delta_{3}$ & $\delta_{4}$ & 1 & 1 & 1 \\
\hline$\delta_{3}$ & $\delta_{1}$ & $\delta_{4}$ & $\delta_{3}$ & $\delta_{7}$ & $\delta_{2}$ & $\delta_{3}$ & 1 & 1 & 1 \\
\hline$\delta_{4}$ & $\delta_{2}$ & $\delta_{3}$ & $\delta_{4}$ & $\delta_{9}$ & $\delta_{4}$ & $\delta_{2}$ & 1 & 1 & 1 \\
\hline$\delta_{5}$ & $\delta_{6}$ & $\delta_{6}$ & $\delta_{6}$ & $\delta_{2}$ & $\delta_{7}$ & $\delta_{5}$ & 1 & $i$ & 1 \\
\hline$\delta_{6}$ & $\delta_{5}$ & $\delta_{6}$ & $\delta_{5}$ & $\delta_{8}$ & $\delta_{8}$ & $\delta_{6}$ & 1 & $i$ & 1 \\
\hline$\delta_{7}$ & $\delta_{7}$ & $\delta_{8}$ & $\delta_{8}$ & $\delta_{3}$ & $\delta_{5}$ & $\delta_{9}$ & $i$ & 1 & 1 \\
\hline$\delta_{8}$ & $\delta_{8}$ & $\delta_{7}$ & $\delta_{7}$ & $\delta_{6}$ & $\delta_{6}$ & $\delta_{0}$ & $i$ & 1 & 1 \\
\hline$\delta_{9}$ & $\delta_{9}$ & $\delta_{9}$ & $\delta_{0}$ & $\delta_{4}$ & $\delta_{9}$ & $\delta_{7}$ & $i$ & $i$ & -1 \\
\hline$\delta_{0}$ & $\delta_{9}$ & $\delta_{0}$ & $\delta_{9}$ & $\delta_{0}$ & $\delta_{0}$ & $\delta_{8}$ & $i$ & $i$ & -1 \\
\hline
\end{tabular}

Table 6: Modular transformations of even spin structures

\section{B.4 The hyperelliptic representation}

Every genus 2 surface admits a hyperelliptic representation, given by a double cover of the complex plane with three quadratic branch cuts supported by 6 branch points, which we shall denote $u_{i}, i=1, \cdots, 6$. The full surface $\Sigma$ is obtained by gluing together two copies of $\mathbf{C}$ along, for example, the cuts from $u_{2 j-1}$ to $u_{2 j}, 1 \leq j \leq 3$. The surface is then parametrized by ${ }^{14}$

$$
s^{2}=\prod_{a=1}^{6}\left(x-p_{a}\right)
$$

\footnotetext{
${ }^{14}$ It is customary to introduce a local coordinate system $z(x)=(x, s(x))$, which is well-defined also at the branch points. Throughout, the formulas in the hyperelliptic representation will be understood in this way. However, to simplify notation, the local coordinate $z(x)$ will not be exhibited explicitly.
} 
In the hyperelliptic representation, there is another convenient way of identifying spin structures. Each spin structure can be viewed then as a partition of the set of branch points $p_{a}, a=1, \cdots, 6$ into two disjoint subsets, in the following way.

$$
\begin{aligned}
\nu \text { odd } & \Leftrightarrow \text { branch point } p_{a} \\
\delta \text { even } & \Leftrightarrow \text { partition } A \cup B, \quad A=\left\{p_{a}, p_{b}, p_{c}\right\}, B=\left\{p_{d}, p_{e}, p_{e}\right\}
\end{aligned}
$$

where $(a, b, c, d, e, f)$ is a permutation of $(1,2,3,4,5,6)$.

\section{B.5 Thomae-type formulas via $\mathcal{M}_{a b}$}

As shown in [4], Thomae relations may be derived most easily in terms of the bilinear $\vartheta$-constants $\mathcal{M}_{a b} \equiv \mathcal{M}_{\nu_{a} \nu_{b}}$ which were defined in [4],

$$
\mathcal{M}_{a b} \equiv \partial_{1} \vartheta\left[\nu_{a}\right](0, \Omega) \partial_{2} \vartheta\left[\nu_{b}\right](0, \Omega)-\partial_{2} \vartheta\left[\nu_{a}\right](0, \Omega) \partial_{1} \vartheta\left[\nu_{b}\right](0, \Omega)
$$

The abbreviation $\partial_{I} \vartheta[\nu] \equiv \partial_{I} \vartheta[\nu](0, \Omega)$ will be convenient. The holomorphic 1-form $\omega_{\nu_{a}}(z)$, defined for any odd spin structure $\nu_{a}$ by $\omega_{\nu_{a}}(z) \equiv \omega_{I}(z) \partial_{I} \vartheta\left[\nu_{a}\right]$, has a unique double zero at $p_{a}$, which is the branch point that may be canonically associated with $\nu_{a}$. The following fundamental results may be derived from a comparison of holomorphic forms in the $\vartheta$ functions and hyperelliptic formulations,

$$
\frac{\mathcal{N}_{\nu_{b}}\left(p_{a}-p_{b}\right)}{\mathcal{N}_{\nu_{c}}\left(p_{a}-p_{c}\right)}=\frac{\omega_{\nu_{b}}\left(p_{a}\right)}{\omega_{\nu_{c}}\left(p_{a}\right)}=\frac{\mathcal{M}_{\nu_{a} \nu_{b}}}{\mathcal{M}_{\nu_{a} \nu_{c}}}
$$

where $\mathcal{N}_{\nu_{a}}$ depends only on $a$ and not on $b$. Taking the cross ratio of four branch points (with $a, d \neq b, c)$, the normalization factors $\mathcal{N}$ cancel out and we get the desired identity

$$
\frac{p_{a}-p_{b}}{p_{a}-p_{c}} \cdot \frac{p_{c}-p_{d}}{p_{b}-p_{d}}=\frac{\mathcal{M}_{\nu_{a} \nu_{b}} \mathcal{M}_{\nu_{c} \nu_{d}}}{\mathcal{M}_{\nu_{a} \nu_{c}} \mathcal{M}_{\nu_{b} \nu_{d}}}
$$

This is a Thomae-type formula, relating $\vartheta$-constants to rational expressions of branch points.

\section{B.6 $\mathcal{M}_{a b}$ in terms of $\vartheta$-constants}

In [4], it was shown that the following relation holds between $\mathcal{M}_{a b}$ and the $\vartheta$-constants for even spin structure,

$$
\mathcal{M}_{a b}=m_{a b} \pi^{2} \prod_{c \neq a, b} \vartheta\left[\nu_{a}+\nu_{b}+\nu_{c}\right]
$$

where $m_{a b}^{2}=1$. The sign factor $m_{a b}$ is not intrinsic, for two reasons. First, $\mathcal{M}_{a b}$ is odd under $a \leftrightarrow b$, while the product of $\vartheta$-constants on the rhs is even. Second, the $\vartheta$-constants 
on the lhs and on the rhs arise to the first power, so that $\mathcal{M}_{a b}$ is actually sensitive to shifts in the $\nu$ 's by single periods (although they are invariant under shifts by any double period). Here, the convention will be taken that the odd spin structures $\nu$ are normalized as given in (B.4), while the even spin structures $\nu_{a}+\nu_{b}+\nu_{c}$ are truncated to the standard normalization given in (B.5),

$$
\nu_{a}+\nu_{b}+\nu_{c} \equiv \delta_{i}, \quad i=0,1, \cdots 9
$$

In the calculation of $\Gamma[\delta ; \varepsilon]$ above, the signs are in fact needed. The sign factors are then given as follows,

$$
\begin{array}{ll}
m_{a b}=-m_{b a} & \\
m_{a b}=+1 & a b=15,23,25,26,35,45,46 \\
m_{a b}=-1 & a b=12,13,14,16,24,34,36,56
\end{array}
$$

The derivation was given in [4]. To obtain the results in the above form, one proceeds as follows. Let $\nu_{0}$ be the unique genus 1 odd spin structure and let $\mu_{1}, \mu_{3}$ and $\mu_{5}$ (and denote an independent set by $\mu_{2}, \mu_{4}$ and $\mu_{6}$ ) denote the three distinct genus 1 even spin structures, obeying $\mu_{1}+\mu_{3}+\mu_{5}=\nu_{0}$, then any pair of genus 2 odd spin structures may be expressed in the following basis,

$$
\nu_{1}=\left[\begin{array}{l}
\mu_{1} \\
\nu_{0}
\end{array}\right] \quad \nu_{2}=\left[\begin{array}{l}
\nu_{0} \\
\mu_{2}
\end{array}\right] \quad \nu_{3}=\left[\begin{array}{l}
\mu_{3} \\
\nu_{0}
\end{array}\right]
$$

The result of [4] is then,

$$
\begin{aligned}
& \mathcal{M}_{\nu_{1} \nu_{2}}=-\pi^{2} \vartheta\left[\begin{array}{l}
\mu_{3} \\
\mu_{2}
\end{array}\right] \vartheta\left[\begin{array}{l}
\mu_{5} \\
\mu_{2}
\end{array}\right] \vartheta\left[\begin{array}{l}
\mu_{1} \\
\mu_{4}
\end{array}\right] \vartheta\left[\begin{array}{l}
\mu_{1} \\
\mu_{6}
\end{array}\right] \\
& \mathcal{M}_{\nu_{1} \nu_{3}}=-\pi^{2} \sigma\left(\mu_{1}, \mu_{3}\right) \vartheta\left[\begin{array}{l}
\nu_{0} \\
\nu_{0}
\end{array}\right] \vartheta\left[\begin{array}{l}
\mu_{5} \\
\mu_{2}
\end{array}\right] \vartheta\left[\begin{array}{l}
\mu_{5} \\
\mu_{4}
\end{array}\right] \vartheta\left[\begin{array}{l}
\mu_{5} \\
\mu_{6}
\end{array}\right]
\end{aligned}
$$

with the sign factor $\sigma\left(\mu_{1}, \mu_{3}\right)=-\sigma\left(\mu_{3}, \mu_{1}\right)$ given as follows,

$$
\sigma\left((0 \mid 0),\left(0 \mid \frac{1}{2}\right)\right)=\sigma\left(\left(\frac{1}{2} \mid 0\right),(0 \mid 0)\right)=\sigma\left(\left(\frac{1}{2} \mid 0\right),\left(0 \mid \frac{1}{2}\right)\right)=+1
$$

Notice that the ordering of $\mu_{1}$ and $\mu_{2}$ determines the sign on the right hand side. Going through all cases, (B.24) is readily derived.

\section{References}

[1] E. D'Hoker and D.H. Phong, "Two-Loop Superstrings I, Main Formulas", Phys. Lett. B529 (2002) 241-255; hep-th/0110247. 
[2] E. D'Hoker and D.H. Phong, "Two-Loop Superstrings II, The chiral Measure on Moduli Space", Nucl. Phys. B636 (2002) 3-60; hep-th/0110283.

[3] E. D'Hoker and D.H. Phong, "Two-Loop Superstrings III, Slice Independence and Absence of Ambiguities", Nucl. Phys. B636 (2002) 61-79; hep-th/0111016.

[4] E. D'Hoker and D.H. Phong, "Two-Loop Superstrings IV, The Cosmological Constant and Modular Forms", Nucl. Phys. B639 (2002) 129-181; hep-th/0111040.

[5] E. D'Hoker and D.H. Phong, "Lectures on two-loop superstrings", Hangzhou, Beijing 2002, hep-th/0211111.

[6] Z. J. Zheng, J. B. Wu and C. J. Zhu, "Two-loop superstrings in hyperelliptic language. I: The main results," Phys. Lett. B 559, 89 (2003) [arXiv:hep-th/0212191]; "Two-loop superstrings in hyperelliptic language. II: The vanishing of the cosmological constant and the non-renormalization theorem," Nucl. Phys. B 663, 79 (2003) [arXiv:hep-th/0212198]; "Two-loop superstrings in hyperelliptic language. III: The four-particle amplitude," Nucl. Phys. B 663, 95 (2003) [arXiv:hep-th/0212219];

[7] C. J. Zhu, "Two-loop computation in superstring theory," arXiv:hep-th/0301018;

W. J. Bao and C. J. Zhu, "Comments on two-loop four-particle amplitude in superstring theory," JHEP 0305, 056 (2003) [arXiv:hep-th/0303152].

[8] E. Martinec, "Non-renormalization Theorems and Fermionic String Finiteness", Phys. Lett. B171 (1986) 189;

R. Iengo and C.J. Zhu, "Notes on Non-renormalization Theorem in Superstring Theories", Phys. Lett. B212 (1988) 309;

O. Lechtenfeld and W. Lerche, "On Non-renormalization Theorems for Four-Dimensional Superstrings", Phys. Lett. B227 (1989) 373;

[9] S. Kachru, J. Kumar, and E. Silverstein, "Vacuum energy cancellation in a nonsupersymmetric string", Phys. Rev D59 (1999) 106004, hep-th/9807076;

S. Kachru and E. Silverstein, "Self-dual nonsupersymmetric Type II string compactifications", JHEP 9811:001 (1998) hep-th/9808056;

S. Kachru and E. Silverstein, "On vanishing two loop cosmological constants in nonsupersymmetric strings", JHEP 9901:004 (1999) hep-th/9810129.48

[10] J. Harvey, "String duality and non-supersymmetric strings", hep-th/9807213;

Z. Kakushadze and S. H. H. Tye, "Brane world," Nucl. Phys. B 548, 180 (1999) [arXiv:hepth/9809147];

G. Shiu and S. H. H. Tye, "Bose-Fermi degeneracy and duality in non-supersymmetric strings," Nucl. Phys. B 542, 45 (1999) [arXiv:hep-th/9808095].

[11] S. Deser and B. Zumino, "Broken Supersymmetry and Supergravity", Phys. Rev. Lett. 38 (1977) 1433;

E. Cremmer, B. Julia, J. Scherk, S. Ferrara, L. Girardello and P. van Nieuwenhuizen, 
"Spontaneous Symmetry Breaking and Higgs Effect in Supergravity without cosmological constant", Nucl. Phys. B147 (1979) 105;

E. Cremmer, S. Ferrara, C. Kounnas and D. V. Nanopoulos, "Naturally Vanishing Cosmological Constant In N=1 Supergravity," Phys. Lett. B133, 61 (1983);

A.B. Lahanas and D.V. Nanopoulos, "The Road to no-scale supergravity", Phys. Rept. 145 (1987) 1;

L. Andrianopoli, R. D'Auria, S. Ferrara and M. A. Lledo, "Gauging of flat groups in four dimensional supergravity," JHEP 0207, 010 (2002) [arXiv:hep-th/0203206].

[12] D. Friedan, E. Martinec, and S. Shenker, "Conformal invariance, supersymmetry, and string theory", Nucl. Phys. B271 (1986) 93.

[13] E. Verlinde and H. Verlinde, "Multiloop calculations in covariant superstring theory", Phys. Lett. B192 (1987) 95-102.

H. Verlinde, "A note on the integral over fermionic supermoduli", Utrecht Preprint No. THU-87/26 (1987) unpublished.

[14] R. Iengo and C.J. Zhu, "Evidence for Non-Vanishing Cosmological Constant in Non-SUSY Superstring Models", JHEP 0004:028 (2000), hep-th/9912074.

[15] E. Gava and R. Iengo, "Modular Invariance and the Two Loop Vanishing of the Cosmological Constant", Phys. Lett. B207 (1988) 283;

C. J. Zhu, "Two Loop Computations In Superstring Theories," Int. J. Mod. Phys. A4 (1989) 3877 .

[16] J. Atick, J. Rabin, and A. Sen, "An ambiguity in fermionic string perturbation theory", Nucl. Phys. B299 (1988) 279-294.

[17] G. Moore and A. Morozov, "Some remarks on two-loop string calculations", Nucl. Phys. B306 (1988) 387-404.

[18] H. La and P. Nelson, "Unambiguous fermionic string amplitudes", Phys. Rev. Lett. 63 (1989) 24-27.

[19] J. Atick, G. Moore, and A. Sen, "Some global issues in string perturbation theory", Nucl. Phys. B308 (1988) 1; "Catoptric tadpoles", Nucl. Phys. B307 (1988) 221-273.

[20] E. D'Hoker and D.H. Phong, "Superholomorphic anomalies and supermoduli space", Nucl. Phys. B292 (1987) 317.

[21] E. D'Hoker and D.H. Phong, "Conformal scalar fields and chiral splitting on super Riemann surfaces", Commun. Math. Physics 125 (1989) 469-513.

[22] R. Dijkgraaf, E. Verlinde, and H. Verlinde, " $c=1$ conformal field theories on Riemann surfaces", Commun. Math. Phys. 115 (1988) 649-690.

[23] E. D'Hoker and D.H. Phong, "The geometry of string perturbation theory", Rev. Modern Physics 60 (1988) 917-1065. 
[24] K. Aoki, E. D'Hoker, and D.H. Phong, "On the construction of some asymmetric orbifold models", to appear.

[25] E. Verlinde and H. Verlinde, "Chiral Bosonization, determinants and the string partition function", Nucl. Phys. B288 (1987) 357;

E. Verlinde and H. Verlinde, "Superstring Perturbation Theory", In Superstrings 88, M. Green et al. Eds., World Scientific Publ. Co. Singapore (1989) 222-240.

[26] R. Russo and S. Sciuto, "Twisted determinants on higher genus Riemann surfaces," Nucl. Phys. B 669, 207 (2003);

R. Russo and S. Sciuto, "Twisted determinants and bosonic open strings in an electromagnetic field," hep-th/0312205.

[27] J. Fay Theta Functions on Riemann surfaces, Springer Lecture Notes in Mathematics, No 352, Springer Berlin (1973).

[28] P. Ginsparg, "Applied Conformal Field Theory", in Les Houches 1988, Session XLIX, fields, strings and critical phenomena, E. Brézin and J. Zinn-Justin eds, North Holland, 1990.

[29] L. Dixon, P. Ginsparg and J.A. Harvey, " $\hat{c}=1$ superconformal field theory", Nucl. Phys. B306 (1988) 470.

[30] A. Erdelyi, ed, Higher Transcendental Functions, Vol II, Bateman Manuscript Project, Krieger Publishing Comp., 1981.

[31] F. Gliozzi, J. Scherk and D. Olive, "Supersymmetry, supergravity theories and the dual spinor model", Nucl. Phys. B122 (1977) 253;

E. Witten, in Symposium on Anomalies, Geometry, Topology, W.A. Bardeen and A.R. White eds, World Scientific (1985) p 61;

N. Seiberg and E. Witten, "Spin structures in string theory", Nucl. Phys. B276 (1986) 272.

[32] M.B. Green, J.H. Schwarz, "Supersymmetrical string theories" Phys Lett B109 (1982) 444.

[33] D.J. Gross, J.A. Harvey, E.J. Martinec and R. Rohm, "Heterotic String Theory (II). The interacting heterotic string", Nucl. Phys. B267 (1986) 75.

[34] E. Martinec, "Superspace geometry of superstrings", Phys. Rev. D28 (1983) 2604;

D. Friedan, "Notes on string theory and two-dimensional conformal field theory", in Unified String Theories: Proceedings of the 1985 Santa Barbara Workshop M.B. Green and D.J. Gross eds, World Scientific 1986;

S. Chaudhuri, H. Kawai and H. Tye, "Path Integral Formulation of Closed Strings", Phys. Rev. D36 (1987) 1148.

[35] E. D'Hoker and D.H. Phong, "Loop amplitudes for the fermionic string", Nucl. Phys. B278 (1986) 225;

G. Moore, P. Nelson, J. Polchinski, "Strings and supermoduli", Phys Lett B169 (1986) 47. 
[36] A. Belavin and V. Knizhnik, "Algebraic geometry and the theory of quantized strings", Phys. Lett. B168 (1986) 201;

[37] E. D'Hoker and D.H. Phong, "Superstrings, Super Riemann Surfaces, And Supermoduli Space," UCLA-89-TEP-32 preprint and in Symposia Mathematica, String Theory, Vol XXXIII, Academic Press London and New York, 1990

[38] K. Aoki, E. D'Hoker and D.H. Phong, "Unitarity of Closed Superstring Perturbation Theory", Nucl. Phys. B342 (1990) 149.

[39] J.I. Igusa, Theta Functions, Springer Verlag, 1972;

J.I. Igusa, "On Siegel modular forms of genus two", Amer. J. Math. 84 (1962) 175; "On the graded ring of theta-constants", Amer. J. Math. 86 (1964) 219.

[40] K.S. Narain, M.H. Sarmidi and C. Vafa, Nucl. Phys. B288 (1987) 551.

[41] R. Dijkgraaf, C. Vafa, E. Verlinde, H. Verlinde, Commun. Math. Phys. 123 (1989) 485.

[42] E. Kiritsis, "Introduction to String Theory", hep-th/9709062;

W. Lerche, A.N. Schellekens and N.P. Warner, Phys. Rept. 177 (1989) 1.

[43] C. Vafa, Nucl. Phys. B273 (1986) 592. 